
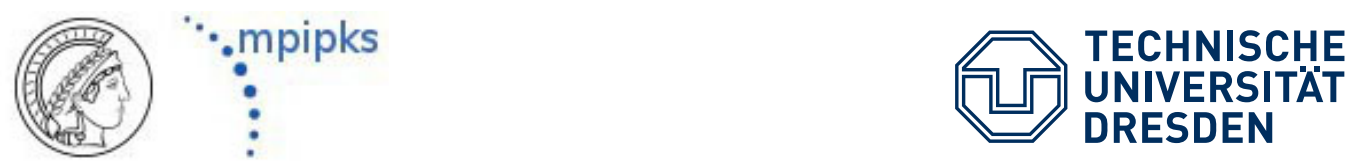

\title{
Emergence and persistence of diversity in complex networks
}

\author{
Dissertation \\ zur Erlangung des akademischen Grades \\ Doctor rerum naturalium \\ (Dr. rer. nat.) \\ vorgelegt von \\ Gesa Angelika Böhme, \\ geboren am 09.03.1985 in München
}

Eingereicht am 19. Dezember 2012

Verteidigt am 4. März 2013 
1. Gutachter: Prof. Dr. Frank Jülicher

2. Gutachter: Prof. Dr. Roland Ketzmerick 


\section{Abstract}

Complex networks are employed as a mathematical description of complex systems in many different fields, ranging from biology to sociology, economy and ecology. Dynamical processes in these systems often display phase transitions, where the dynamics of the system changes qualitatively. In combination with these phase transitions certain components of the system might irretrievably go extinct. In this case, we talk about absorbing transitions. Developing mathematical tools, which allow for an analysis and prediction of the observed phase transitions is crucial for the investigation of complex networks.

In this thesis, we investigate absorbing transitions in dynamical networks, where a certain amount of diversity is lost. In some real-world examples, e.g. in the evolution of human societies or of ecological systems, it is desirable to maintain a high degree of diversity, whereas in others, e.g. in epidemic spreading, the diversity of diseases is worthwhile to confine. An understanding of the underlying mechanisms for emergence and persistence of diversity in complex systems is therefore essential. Within the scope of two different network models, we develop an analytical approach, which can be used to estimate the prerequisites for diversity.

In the first part, we study a model for opinion formation in human societies. In this model, regimes of low diversity and regimes of high diversity are separated by a fragmentation transition, where the network breaks into disconnected components, corresponding to different opinions. We propose an approach for the estimation of the fragmentation point. The approach is based on a linear stability analysis of the fragmented state close to the phase transition and yields much more accurate results compared to conventional methods.

In the second part, we study a model for the formation of complex food webs. We calculate and analyze coexistence conditions for several types of species in ecological communities. To this aim, we employ an approach which involves an iterative stability analysis of the equilibrium with respect to the arrival of a new species. The proposed formalism allows for a direct calculation of coexistence ranges and thus facilitates a systematic analysis of persistence conditions for food webs.

In summary, we present a general mathematical framework for the calculation of absorbing phase transitions in complex networks, which is based on concepts from percolation theory. While the specific implementation of the formalism differs from model to model, the basic principle remains applicable to a wide range of different models. 


\section{Zusammenfassung}

Komplexe Netzwerke werden zur mathematischen Beschreibung komplexer Systeme in vielen Bereichen eingesetzt, angefangen von der Biologie, über die Soziologie und Wirtschaft, bis hin zur Ökologie. Dynamische Prozesse in solchen Systemen weisen oft Phasenübergänge auf, bei welchen sich die Dynamik des Systems qualitativ ändert. Im Zusammenhang mit derartigen Phasenübergängen kann es vorkommen, dass bestimmte Komponenten des Systems unwiederbringlich verloren gehen. In diesem Fall sprechen wir von einem absorbierenden Übergang. Die Entwicklung mathematischer Methoden zur Analyse und Vorhersage der beobachteten Phasenübergänge ist entscheidend für die Untersuchung von komplexen Netzwerken.

In dieser Arbeit behandeln wir absorbierende Übergänge in dynamischen Netzwerken, in welchen Diversität verloren geht. In manchen Beispielen aus der realen Welt, z.B. in der Entwicklung einer Gesellschaft oder eines Ökosystems, ist es wünschenswert ein hohes Maß an Diversität zu erhalten, während es in anderen, z.B. bei der Ausbreitung von Krankheiten, erstrebenswert ist die Diversität an Erkrankungen einzudämmen. Dafür ist ein Verständnis der zu Grunde liegenden Mechanismen für die Enstehung und das Bestehen von Diversität essentiell. Im Rahmen zweier verschiedener Netzwerk Modelle entwickeln wir einen analytischen Ansatz, der verwendet werden kann um notwendige Voraussetzungen für Diversität abzuleiten.

Im ersten Teil betrachten wir ein Modell, das Meinungsbildungsprozesse in einer Gesellschaft beschreibt. In diesem Modell sind Phasen geringer Diversität von Phasen hoher Diversität durch einen Fragmentierungsübergang getrennt, in welchem das Netzwerk, entsprechend den unterschiedlichen Meinungen, in separate Komponenten zerfällt. Wir entwickeln einen Ansatz für die Bestimmmung des Fragmentierungspunktes. Der Ansatz basiert auf einer linearen Stabilitätsanalyse des fragmentierten Zustands in der Nähe des Phasenübergangs und liefert wesentlich genauere Ergebnisse im Vergleich zu konventionellen Methoden.

Im zweiten Teil betrachten wir ein Modell, das die Enstehung von komplexen Nahrungsnetzen beschreibt. Wir berechnen Koexistenzbedingungen für verschiedene Typen von Spezies in einer ökologischen Population. Dafür verwenden wir einen Ansatz, der eine schrittweise Stabilitätsanalyse des Gleichgewichtszustandes vor der Ankunft einer neuen Spezies beinhaltet. Der Formalismus erlaubt eine unmittelbare Berechnung der Koexistenzbereiche von Nahrungsnetzen und ermöglicht somit eine systematische Untersuchung der Bedingungen für deren Erhalt.

Insgesamt formulieren wir eine mathematische Vorgehensweise für die Berechnung von absorbierenden Phasenübergängen in komplexen Netzwerken, welche auf Konzepten aus der Perkolationstheorie basiert. Während sich die jeweilige Umsetzung unseres Ansatzes von Modell zu Modell unterscheidet, lässt sich das Grundprinzip auf einen weiten Bereich unterschiedlicher Modelle übertragen. 


\section{Contents}

$\begin{array}{llr}1 & \text { Introduction } & 1\end{array}$

2 Basic concepts of network theory 5

2.1 Network structure . . . . . . . . . . . . . . 5

2.1.1 Properties of networks . . . . . . . . . . . . 6

2.1.2 Network models . . . . . . . . . . . . . . . . . . 9

2.2 Network dynamics . . . . . . . . . . . . . . . . . . . . 11

2.2.1 Dynamics of networks . . . . . . . . . . . 11

2.2 .2 Dynamics on networks . . . . . . . . . . . . 12

2.2 .3 Adaptive networks . . . . . . . . . . . . . . . . 14

2.3 Network analysis . . . . . . . . . . . . . . . . . . 15

2.3.1 Reduction of the state space . . . . . . . . . . 15

2.3.2 Tools from dynamical systems theory . . . . . . . . . . 19

3 Fragmentation transitions in models for opinion formation 22

3.1 The adaptive voter model . . . . . . . . . . . . . . . 23

3.1 .1 Definition of the model . . . . . . . . . . . 23

3.1.2 Conventional calculation of the fragmentation transition 25

3.2 Percolation approach for the calculation of fragmentation thresholds 27

3.2.1 Basis of $q$-fans . . . . . . . . . . . . . . . . . . . . . . 28

3.2 .2 Basis of spider motifs . . . . . . . . . . . . . . . 32

3.3 Fragmentation transitions in multi-state voter models . . . . . . 35

3.3.1 Definition of the multi-state voter model . . . . . . . . 36

3.3.2 Fragmentation transitions in a 3 -state model . . . . . . . 37

3.3.3 Fragmentation transitions in a $G$-state model . . . . . . 45

3.4 Further applications of the percolation approach . . . . . . . 53

3.4.1 Directed voter model . . . . . . . . . . . . . . 53

3.4 .2 Estimation of active link density . . . . . . . . . . . 56

3.5 Discussion . . . . . . . . . . . . . . . . . . . 59

4 Persistence of complex food webs in metacommunities 62

4.1 Introduction to metacommunity theory . . . . . . . . . . . 63

4.1.1 Definition of the model . . . . . . . . . . . . . 63

4.1.2 Persistence conditions for food chains . . . . . . . . 65

4.2 Influence of the food web on the persistence conditions . . . . . 68

4.2.1 Two simple food webs involving omnivores . . . . . . 68

4.2.2 Omnivores feeding on two arbitrary prey species . . . . . 76

4.2.3 Omnivores feeding on more than two prey species . . . . 82 
4.3 Influence of the patch-network on the persistence conditions . . 87

4.3.1 Patch-networks with multiple types of habitat . . . . . 87

4.3.2 Patch-networks with heterogeneous degree distributions . 92

4.4 Discussion . . . . . . . . . . . . . . . . . . . 96

$\begin{array}{llr}5 \text { Conclusion } & 99\end{array}$ 


\section{Introduction}

In our world of ongoing globalization we are experiencing an increasing diversity in many aspects. Starting with the broad variety of different products in supermarkets, to a wide range of newspapers and media channels in different languages and, not at last, to the incredible amount of options the internet offers, diversity has become an ubiquitous feature in our every-day life. Undoubtedly, this development is a great achievement, as the coexistence of many different opinions, languages and traditions constitutes an enrichment for the human society and promotes exchange and, desirably, tolerance. At the same time, it can be observed that an increased exchange between continents and cultures leads to a loss of traditions, to disappearance of languages or to extinction of species [1-3]. Therefore, a major challenge consists in maintaining diversity in a more and more "connected" world.

It is evident that not only human society, but nature itself displays an exceptional degree of diversity at any level of observation. For example, there are many different proteins and cell types, a great variety of microorganisms and organisms and an immense number of different ecological species. Considering this biological diversity, one can ask why there are so incredibly many variations in nature. While there is certainly no exhaustive answer to this question, the advantages of diversity are rather obvious: first of all, different components can accomplish different functions, so that a division of labour is realized, which makes, for example, a cellular process much more efficient [4]. A second positive aspect is an enhanced resilience. For this reason, in many technical systems redundant diversity is intentionally implemented in order to minimize sensitivity against attacks $[5,6]$. So, one can state that diversity, apart from being "exciting" and "interesting", comprises benefits in terms of efficiency and stability for a system.

Next, one can ask how the coexistence of these incredibly many variations in nature is achieved, i.e. what are the prerequisites to facilitate the emergence and persistence of diversity in a system? This leads directly to the central question of the present work. Here, we mathematically explore the conditions which are required for a system in order to exhibit diversity. Understanding the principles for the development of diversity constitutes not only a fundamental ingredient for studying evolution and functioning of these systems, but it is also essential for maintaining diversity in given systems and for engineering diversity in artificial systems.

The systems we are referring to are so-called complex systems. All the examples mentioned above, from single cells to systems of cells and from ecological systems to human societies, represent complex systems. There exist many different definitions for the term complexity, depending on the context and the field $[7,8]$. Concerning complex systems, it is widely agreed upon, that these 
are systems which comprise one common characteristic, namely the emergence of collective properties [9-11]. This means, complex systems exhibit features which emerge as a result of the local interactions between many interacting components. Remarkably, the emergent features are not present at a local scale, i.e. they are not inherent to the individual components, but rather emerge at the system level, due to the aggregate interactions.

Examples for emergent phenomena are synchronization, ordering and swarming processes [12-14], or the formation of patterns, of complex, hierarchical structures and distinct communities [15-17]. While the first examples suggest, that emergent properties tend to blur all distinctions at the individual level, the latter ones indicate that also the opposite can happen, namely diversity can arise in an initially homogeneous system.

Intriguingly, there are also systems which display both, regimes of diversity and regimes of homogeneity, separated by a phase transition. A transition to a state of lower diversity implies the extinction of part of the initial amount of components in the system. These phase transitions are therefore called $a b$ sorbing transitions. In real-world situations, absorbing transitions often imply profound changes, as they lead to irretrievable loss of species, languages, traditions or opinions. Conversely, such transitions can be beneficial, when for example infectious diseases, vermin or criminality go extinct. Because of their particular importance for real-world applications absorbing transitions are the major concern of the present work. Our goal is to identify the conditions which have to be imposed on the parameters of the system, in order to drive the system into a specific regime.

A convenient representation of complex biological, technological or social systems can be obtained by introducing networks [18-21]. A network description involves a reduction of the system's components to nodes and a reduction of the interactions between the components to links, connecting the nodes. Naturally, in a network description many details of the original system are neglected. However, the simplification still captures the essential features and facilitates a mathematical analysis. For example, structural properties of the derived network can provide insight into functioning and failure of the system under consideration, or allow for an identification of critical and redundant components of the system.

As most complex systems in nature are not static, but underly constant evolution and rearrangement, one typically has to deal with dynamical networks. The large number of degrees of freedom and the typically stochastic and nonlinear nature of the dynamical processes, make the analysis of large dynamical networks difficult. Computer simulations of the microscopic dynamics, though in principle viable, often require immense computational effort and give little insight into general mechanisms. Therefore, one is interested in developing feasible analytical methods to describe dynamics in complex networks. A common ap- 
proach is the derivation of a low-dimensional system of equations which captures the dynamics of macroscopic quantities and thus provides valuable information about statistical properties and equilibrium states of the system [18, 22, 23]. In particular, it allows for the investigation of phase transitions, characterized by an abrupt change in the nature of the equilibrium states. One challenge in this approach is to find adequate macroscopic variables, which faithfully predict the global behavior of the full system.

In the present work we consider network models which exhibit both, homogeneous phases and phases of considerable diversity. Investigating the requirements for diversity, our goal is to determine the conditions where phases of high diversity appear or disappear, respectively. To this aim we develop a mathematical approach for the calculation of phase transitions in network models. The approach allows for the estimation of the parameter ranges where diversity emerges, and those regions in parameter space where diversity is lost.

We demonstrate our approach using two different classes of models. The first model describes opinion formation in a human society, while the second model describes the formation of ecological communities. Although the specific procedure depends on the type of the model and, in particular, on the nature of the phase transition, the general idea is similar for both cases: We study percolation in a state close to the phase transition. Then, using linear stability analysis, we identify the conditions where the steady state becomes unstable, corresponding to a percolation threshold. We argue that the proposed approach can be applied to a wide range of different models.

The outline of the present work is the following: In Chapter 2, we give a glimpse into complex network theory and dynamical systems theory, providing a methodological basis for the subsequent chapters. We start with a brief overview of the basic definitions in complex networks theory and introduce the most important properties of real-world networks and network models. Then, we discuss dynamical processes in networks and provide methods for the analysis of dynamical networks.

In Chapter 3, we discuss a paradigmatic model for opinion formation in a human society, a so-called adaptive voter model. The model exhibits a phase transition between a globally homogeneous state and a state where several opinions coexist within separated subgroups of the system. Common analytical approaches fail in predicting this phase transition faithfully. In this work we propose an alternative analytical approach for the calculation of the phase transition in the adaptive voter model, which yields a very good agreement with simulation results. We first explain our approach in detail for the simplest version of the model and then extend our analysis to more sophisticated variants of the model. For example, we consider an extension of the model to an arbitrary number of states. For this multi-state voter model we obtain a phase diagram in 
which three distinct regimes occur. Furthermore, our calculations reveal classes of equivalent systems in the space of all multi-state voter models, i.e. systems which behave similarly with respect to certain phase transitions. The crucial point of our approach is the choice of an appropriate basis set for the derivation of low-dimensional equations, constituting the main result of this chapter.

In Chapter 4, we study the formation of complex food webs in ecological communities, employing a so-called metacommunity model. We develop a mathematical framework which allows for the computation of the parameter range, where several (competing) species coexist in the system. Then, we analyze the dependence of this parameter range on the interactions between species, the food web, and on the properties of the underlying landscape, the network of resource-patches. The main achievement of this chapter constitutes a generalized framework for the calculation of coexistence ranges for ecological species, applying an approach which originates in the calculation of epidemic thresholds.

In Chapter 5, we summarize the main results obtained in the course of this work. We point out that the mathematical formalisms developed here uses a simple, but powerful concept to obtain analytical results for absorbing transitions in network models, which makes it a promising tool for the analysis of existing and future models from different fields. 


\section{Basic concepts of network theory}

In this chapter, we give a short introduction into the terminology and the basic concepts of network theory. Furthermore, we provide an outline of dynamical processes in networks and discuss some important tools for the analysis of such processes.

We note that the selection and the scope of the presented topics do not constitute an extensive survey of network theory or dynamical systems theory. The aspects discussed here rather aim to provide a reasonable background for understanding and assessing the specific problems and results presented in this work. For a broader analysis of the subject, we refer to comprehensive textbooks as e.g. [18, 22, 24-26].

Further, we note that some of the theoretical concepts introduced here, although mathematically sound, are not presented in a mathematically rigorous way. We rather try to illustrate the definitions and approaches in a way that is accessible for the nonspecialist reader and that emphasizes the general idea. References to the corresponding profound literature are provided.

We start in Section 2.1 with basic definitions concerning the structure and properties of networks and introduce the most important network models. In Section 2.2 we discuss dynamics in networks. We illustrate different types of dynamical processes using a simple example model. In Section 2.3 we provide some central mathematical concepts for the analysis of network dynamics.

\subsection{Network structure}

A network is an abstract description of a complex system comprising many interacting components. The central aim of network theory is to understand the functioning and failure of complex systems in nature. Often, the function of these systems is closely related to the interaction pattern of the components, i.e. the underlying network structure. In order to compare and classify different types of networks with respect to their structural properties, a quantitative description of networks is sensible. Based on the main characteristics of real-world networks, i.e. networks representing biological, social or technological systems in nature, one can then construct network models which resemble the observed properties.

In the following we present the most important characteristics of networks and their quantification. Then, we introduce commonly used network models and discuss their properties. This section is mainly based on [18, 20, 27]. 


\subsubsection{Properties of networks}

Describing a complex system as a network implies a reduction of its components to nodes and a reduction of the interactions between the components to links between the nodes. Then, the system can be depicted as a graph with a given number of nodes $N$ and a given number of links $L$ (see Fig. 2.1 for examples). We will refer to the size of a network by the total number of nodes, $N$, it is composed of.

Two nodes which are connected via a link are said to be neighboring, or adjacent nodes. A path is a sequence of nodes, where consecutive nodes in the sequence are adjacent and every node in the sequence appears only once. A cycle is a closed path, i.e. a sequence of at least three adjacent nodes where the first and the last node in the sequence coincide. In this work we consider simple graphs. This means, we do not allow for multiple links connecting the same pair of nodes and we do not allow for loops, i.e. links going from a node to itself.

A specific graph is completely defined by its adjacency matrix, a $N \times N$-matrix A with elements

$$
A_{i j}= \begin{cases}1 & \text { if there is a link from node } i \text { to node } j \\ 0 & \text { if there is no link from node } i \text { to node } j\end{cases}
$$

If $\mathbf{A}$ is symmetric, the corresponding graph is undirected, otherwise the graph is directed. A directed graph represents unidirectional relationships between neighboring nodes. For example food webs, which describe predator-prey relationships, or gene regulatory networks, which describe activating and inhibitory interactions between proteins, are represented by directed graphs. On the contrary, networks of human acquaintances or many technological networks are usually assumed bidirectional and are therefore described by undirected graphs.

Depending on the system under consideration, some links might have more importance than others, giving rise to link weights. In this case, the entries of the corresponding adjacency matrix $\mathbf{W}$ assume values $w_{i j}$ (in general $w_{i j} \neq 1$ ) if $i$ is connected to $j$, and 0 otherwise. For illustration of the different graph types, two simple example graphs and their corresponding adjacency matrices are given in Fig. 2.1.

The number of neighbors of a node $i$ is called the degree $k_{i}$ of the node. Given the adjacency matrix of a graph, the degree of a node $i$ can be calculated as

$$
k_{i}=\sum_{j} A_{i j}=\sum_{j} A_{j i} .
$$

In a directed network, as $A_{i j} \neq A_{j i}$, it follows that in general the out-degree $k_{i}^{\text {out }}$, corresponding to the number of links emanating from node $i$, and the in-degree $k_{i}^{i n}$, corresponding to the number of links arriving at node $i$, are different. Out- 


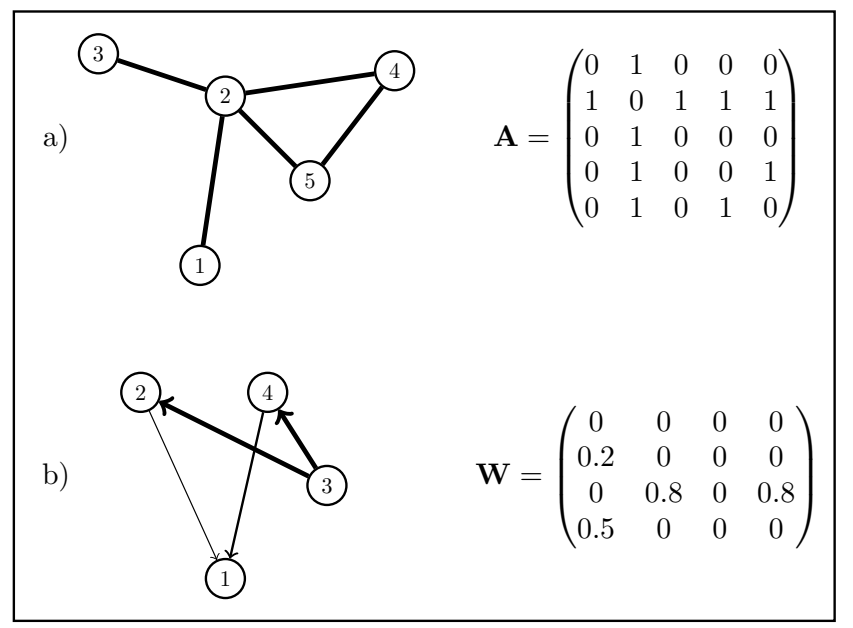

Figure 2.1: Examples for simple graphs. Shown are a) an undirected, unweighted graph and b) a directed, weighted graph with the corresponding adjacency matrices. Nodes are depicted as circles, undirected links as lines and directed links as arrows. Different arrow widths correspond to different weights. The nodes are numbered corresponding to their position in the adjacency matrix.

and in-degree of a node $i$ are accordingly given by,

$$
k_{i}^{\text {out }}=\sum_{j} A_{i j}, \quad k_{i}^{i n}=\sum_{j} A_{j i} .
$$

After this brief description of the main building blocks of a network we now turn to the most important statistical properties, which allow for a certain distinction and classification of networks. It is obvious that two networks, though comprising equal numbers of nodes and links, in general differ with respect to their structure. A characterization of the structural properties of a network often provides information about the functioning of a related real-world system and becomes crucial when considering dynamics on networks, as we will see later.

One important network property is the degree distribution, which captures the probability $P(k)$ that a randomly chosen node has degree $k$. The mean degree of a network is fully determined by the total number of nodes and links,

$$
\langle k\rangle=\sum_{k} k P(k)=\frac{1}{N} \sum_{i} k_{i}=\frac{2 L}{N} .
$$

The degree distribution quantifies the heterogeneity of nodes with respect to their connectivity. 
We talk about a degree-homogeneous network if the degree distribution is a narrow function in $k$, or more precisely, if the probability for a node to have a degree $k>\langle k\rangle$ decreases exponentially with increasing deviation from the mean degree $^{1}$. An extreme case is a degree-regular network where every node has exactly the same degree and the degree distribution is a $\delta$-function. A complete graph, where all possible links are realized, is also a degree-regular graph.

In a degree-heterogeneous network, in contrast, the probability to find a node with $k>\langle k\rangle$ decreases slower than exponentially, implying that a significant amount of nodes with very high degrees exists. Heterogeneous networks are of particular interest for applications, as many real-world networks exhibit broad degree distributions [20, 21]. A characteristic feature of such degree distributions is a scale-free behavior, described by a probability function $P(k) \sim k^{-\gamma}$. Expressed in words, this means that most of the nodes have very few links, while few nodes have a large percentage of all links. The extreme case of a heterogeneous network is a star graph, where the focal node connects to all the remaining nodes in the network, while each of the fringe nodes has only a single connection.

A degree-related quantity is the (degree)-assortativity, which captures correlations between the degrees of neighboring nodes. An (in average) positive correlation is called assortative and implies that nodes with high degree are most likely connected to other high-degree nodes, while nodes with low degree tend to connect to other low-degree nodes. An (in average) negative correlation is called disassortative and refers to networks where high-degree nodes are typically attached to low-degree nodes. Social networks tend to be assortative, while technological and biological networks are disassortative [28]. Degree correlations can be quantified via the conditional probability $P\left(k^{\prime} \mid k\right)$, which denotes the probability for a node of degree $k$ to have a neighboring node of degree $k^{\prime}$. In the absence of degree correlations the conditional probability becomes

$$
P\left(k^{\prime} \mid k\right)=\frac{k^{\prime} P\left(k^{\prime}\right)}{\langle k\rangle}
$$

Equation (2.1) implies that the probability to find a node of degree $k^{\prime}$ by following a random link increases with $k^{\prime}$. So, it is likely to pick a node of high degree by following a random link. This can be intuitively understood, as there are $k^{\prime}$ possibilities to reach a node of degree $k^{\prime}$. Accordingly, when considering dynamical updates in networks it makes a difference whether one picks a node or a link at random, in one update step (see e.g. [29]).

Another important characteristic of networks is the transitivity, or clustering. There exist many different ways of quantifying clustering. Here, we introduce

${ }^{1}$ The same applies for $k<\langle k\rangle$, but as the degree is bounded from below, we are mainly interested in the upper tail of the distribution. 
the clustering coefficient as (see e.g. [20])

$$
C=\frac{3 \times \text { number of triangles }}{\text { number of triplets }}
$$

where a triplet is a subgraph of the network consisting of three nodes which are joined by at least two links, and triangles are cycles of three nodes. In other words, $C$ quantifies the probability that two neighbors of a node are connected. Social networks typically show high clustering coefficients, which supports the statement "the friend of a friend is a friend".

The last property we mention here is the average shortest path length $\langle l\rangle$, which describes the reachability of any node in the network from a given node. The length of the shortest path between two nodes $i$ and $j$ in the network is the minimum number of links, one has to follow in order to reach node $j$, starting at node $i$. Then, the average is taken over all possible pairs of nodes.

A small average shortest path length compared to the network size has become known as the small-world property, suggesting that any pair of nodes is relatively "close" to each other, despite of a large total number of nodes in the network. Quantitatively, this means that the average shortest path length scales logarithmically with the network size: $\langle l\rangle \sim \ln N$ [30]. The pioneering experiment which measured the shortest path length in human societies was conducted by Milgram in 1967 [31]. He found that the average shortest path length in a network of acquaintances is $\langle l\rangle=6$, coining the expression "six degrees of separation". This phrase became a popular synonym for the small-world property, though it only refers to the small average path length and does not reflect the typically high clustering coefficient in small-world networks.

\subsubsection{Network models}

For studying real-world systems it is not only essential to analyze the properties of their underlying network structure, but also to build artificial networks which reproduce features observed in real-world networks. To this aim, a variety of network models has been proposed. In the following we briefly discuss three of them, which are widely used in the networks literature and highlight their respective characteristic properties. Example graphs of the three construction algorithms are shown in Fig. 2.2.

First, let us consider the Erdös-Rényi random graph model [32]. Starting from a given number of nodes $N$, every pair of nodes is connected with probability $p$. The mean degree of the resulting network is related to the probability $p$ through $\langle k\rangle=p(N-1)$. The degree distribution can be described by a Poisson distribution,

$$
P(k)=\left(\begin{array}{c}
N \\
k
\end{array}\right) p^{k}(1-p)^{N-k} \cong \frac{\langle k\rangle^{k} e^{-\langle k\rangle}}{k !},
$$


where the last equality is valid in the limit of large $N$ and for fixed mean degree. By construction, there are no degree-correlations between degrees of neighboring nodes and clustering tends to 0 for large $N(C \sim 1 / N)$ [33]. Erdős-Rényi random graphs display the small-world property, as $l \sim \ln N / \ln \langle k\rangle$ [34]. The ErdösRényi model has been widely used because of its simplicity and mathematical tractability. Although the properties might not reflect all the features observed in real-world networks, the results obtained using Erdős-Rényi random graphs provide valuable insights and serve as reference point for more realistic models.

As a low average shortest path length and a high clustering coefficient turned out to be characteristic for social networks, the network model introduced by Watts and Strogatz [30,33] aimed to combine these two properties. Watts and Strogatz proposed to start with a periodic regular lattice, in the simplest case a ring of $N$ nodes, where every node is connected to its $m$ nearest neighbors. Then, each link is randomly rewired with probability $p$. The degree of randomness can be tuned via the parameter $p$, which is accompanied by an interpolation between graphs with high clustering ( $p=0$, regular lattice) and graphs with small average path length ( $p=1$, random graphs). Numerical simulations show that there is an intermediate regime where both, a small average path length and high clustering are realized [30].

Finally, in order to obtain networks with more realistic degree distributions, the Barabási-Albert model was introduced [35]. The construction algorithm is the following: Starting with a small number of connected nodes $m_{0}$, in every time-step a new node $i$ with $m \leq m_{0}$ links is added. One of the new links is connected with probability

$$
p_{j}=\frac{k_{j}}{\sum_{i} k_{i}}
$$

to an already existing node $j$. This procedure is continued until a maximal number of nodes $N$ is reached. Because the probability for a node $j$ to gain a new link is proportional to the actual degree of the node, $k_{j}$, the link attachment process is called preferential attachment. Growth and preferential attachment lead to a network with scale-free degree distribution for large $N$. More precisely, the emerging degree distribution follows $P(k) \sim k^{-\gamma}$ with $\gamma=3$, which makes the Barabási-Albert model a favored candidate model for many real-world applications. In the Barabási-Albert model, degree correlations are mainly present between "old" and "new" nodes, i.e. between nodes which entered the network at an early stage and those that were added at a later time-step. Degree correlations between randomly chosen pairs of nodes vanish for $N \rightarrow \infty$ [28].

Although none of the three described models captures all the properties which are observed in real-world networks, each of them reproduces some relevant aspects. So, altogether, the presented network models serve as a useful toolbox for the development of more realistic models and comprise toy models for testing 
a) Erdős-Rényi

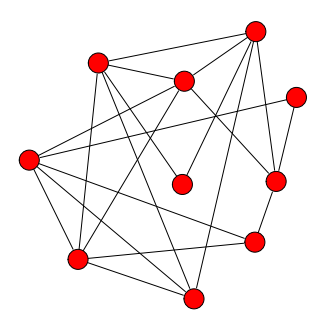

b) Watts-Strogatz

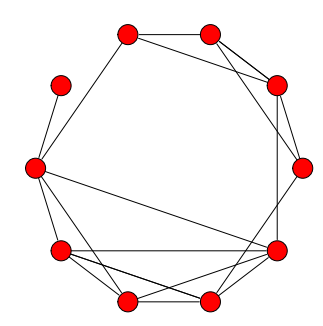

c) Barabási-Albert

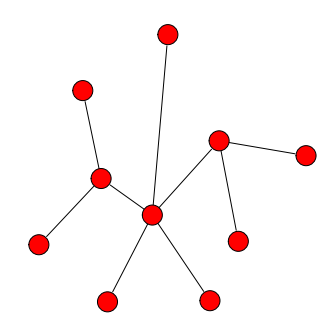

Figure 2.2: Illustration of three different network models. Shown are small example networks constructed according to the algorithms described in the text. Parameters: $N=10$, a) $L=20$, b) $m=2, p=0.2$, c) $m_{0}=1, m=1$.

analytical methods and running simulations. In particular, these models provide means for studying the influence of the network structure on the network dynamics. While so far, we considered static networks, it is evident that biological, social and technological systems underly constant changes, which have to be described within a framework of dynamical networks. In the following section we discuss dynamical processes in networks.

\subsection{Network dynamics}

The term "network dynamics" includes two types of dynamics, namely dynamics of the network and dynamics on the network. In general, a network can display both types of dynamics. In particular, the dynamics of the network and the dynamics on the network can occur to be coupled. In this case, the network is called an adaptive network.

\subsubsection{Dynamics of networks}

Dynamics of the network refers to the evolution of the network structure, i.e. to an evolving network topology. Mathematically, the dynamics of the network becomes manifest in a time-dependent adjacency matrix $\mathbf{A}(t)$. If one is interested in the evolution of the network structure rather than in the final topology, the network models discussed in the last section can actually be seen as dynamic models. Apart from these models, one can think of processes where links are deleted, added or rewired according to some given rule. As rewiring rules leave the number of links constant, they are better to treat analytically.

While studies of evolving network topologies mainly focus on network growth $[36,37]$ and preferential attachment [38-40], the investigation of dynamics on 
networks covers a variety of different processes, ranging from spreading and percolation to synchronization and cooperation processes on networks (see [18] and references therein). In the following section we study dynamics on the network in more detail and discuss a simple model for epidemic spreading as an example.

\subsubsection{Dynamics on networks}

Dynamics on the network refers to a dynamical process taking place on an underlying (static) network structure. This type of dynamics can be captured by introducing node states ${ }^{2}$, i.e. assigning variables $\sigma_{i}(t)$ to the network nodes, which are time-dependent. The node states are drawn from a discrete set or a continuous range; in some cases the node states are vectors. In this work, we consider scalar and discrete states.

For example, in a computer network the spreading of a computer virus is a dynamical process where the nodes correspond to computers which assume states from a discrete set $\{H, I\}$. Switching from a "healthy" state $\left(\sigma_{i}=H\right)$ to an "infected" state $\left(\sigma_{i}=I\right)$ can be due to a contamination caused by a connected computer in the network, by an external source, or by some intrinsic failure.

In general, we call node state dynamics that involve interactions with neighboring nodes contact processes. Although there might be additional intrinsic and external influences affecting the node states, the contact process is the only one which depends on the network topology. So actually, when we talk about dynamics on networks, at least some kind of contact process is involved. However, in a broader sense, a contact process is not necessarily a local interaction occurring between nearest neighbors, but it can involve long-range interactions, e.g. between next nearest neighbors.

One example where contact processes become relevant in real-world systems is the spreading of a disease. While individuals can catch a disease spontaneously with a certain probability, an epidemic outbreak becomes only possible because infected individuals pass the disease to their social environment, their neighbors in the network. The study of epidemic spreading has been one of the main concerns since the very beginnings of network research [41-44], offering a promising fields where results from network theory can be used for the development of vaccination strategies and containment of diseases. However, only recently, since data of international traffic networks, computer networks and social networks is available for testing the theoretical results obtained in models, the ultimate

\footnotetext{
${ }^{2}$ In principle, one can also account for link states, giving rise to (additional) dynamics. In the present work, however, we do not explicitly consider different link states, but rather define the state of a link through the states of the nodes it connects to. Consequently, we regard potential changes in link states as secondary effects of the node state dynamics.
} 
goal of understanding and controlling epidemic outbreaks seems to come into reach $[45-47]$.

As an example for a dynamical process taking place on a network, let us in the following briefly discuss a simple and paradigmatic model for epidemic spreading, the $S I S$-model [41]. The letter $S$ stands for the "susceptible" and the letter $I$ for the "infectious" state. In this model network nodes correspond to individuals which assume states from the set $\{S, I\}$, according to their condition. The network links correspond to contacts between individuals. Susceptible individuals can be infected by infectious neighbors with a rate $\beta$, whereas infectious individuals recover with a rate $\gamma$, independent of their neighborhood, and become susceptible again. These two processes are captured in the following transition equations:

$$
\begin{gathered}
S+I \stackrel{\beta}{\rightarrow} I+I, \\
I \stackrel{\gamma}{\rightarrow} S .
\end{gathered}
$$

The crucial question is, whether an epidemic can invade and persist in the system. The corresponding characteristic parameter is the epidemic threshold $\epsilon$, beyond which an epidemic outbreak becomes possible. For degree-homogeneous networks, the epidemic threshold is given by $1 /\langle k\rangle$. This means, for

$$
\frac{\beta}{\gamma}<\epsilon=\frac{1}{\langle k\rangle}
$$

an epidemic outbreak is prevented. For scale-free degree distributions the epidemic threshold is given by the maximal eigenvalue of the adjacency matrix, $\epsilon=\lambda(\mathbf{A})$, implying that the epidemic threshold vanishes for infinite network size. Thus, an epidemic can always persist in a scale-free network, though at low prevalence level [48, 49].

The $S I S$-model and many of its variants have been extensively studied in the past decade [48-53]. Thereby, the model represents not only a paradigmatic model for its original purpose, the spreading of diseases, but its applications extend to other fields where spreading processes are important, such as information and rumor spreading in social networks and online communities [54-57]. Furthermore, refined versions of the SIS-model, so-called compartmental models consider more than two states $[58,59]$ and are closely related to metacommunity models in ecology, which will be discussed in Chapter 4.

One of the most intriguing results for epidemic spreading involves a mapping to percolation processes on networks [60,61]. As percolation on regular lattices had been thoroughly studied in statistical physics, concepts from percolation theory were extended to complex networks and to the study of dynamical processes on networks. The analytical approach we develop in Chapter 3 for the 


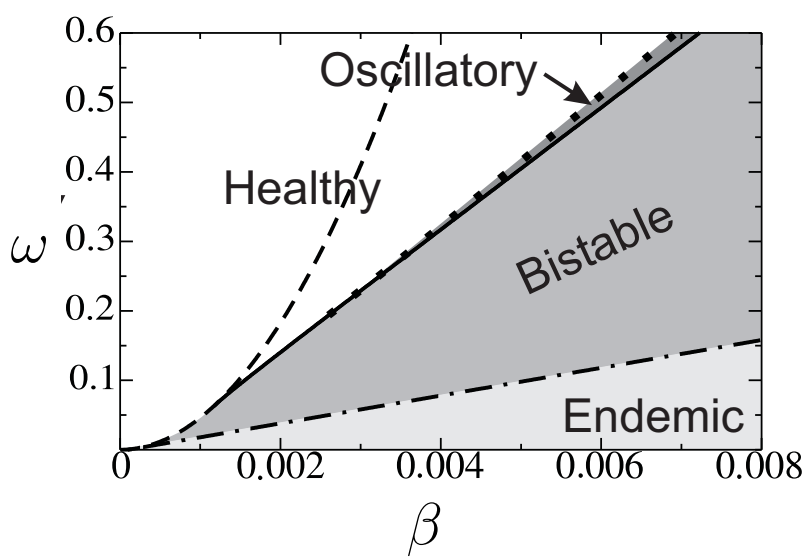

Figure 2.3: Phase diagram for the adaptive SIS-model (figure adapted from [72]). Depending on the rewiring rate $\omega$ and the infection rate $\beta$ (recovery rate $\gamma=0.002$ ) healthy, endemic, oscillatory or bistable regimes are observed. Phase diagrams of this kind, offering a multitude of different dynamical regimes are a common feature of adaptive networks.

calculation of fragmentation transitions, as well as the mathematical framework provided in Chapter 4 for the calculation of persistence ranges are inspired by the idea of percolation.

In this section, we considered dynamics on networks, assuming a static network topology. In many systems in nature, however, the network itself, i.e. the pattern of interactions, evolves in time. Moreover, the network topology does not change independently of the network nodes, but can be affected by the current state of the network nodes. Conversely, the evolution of the node states depends on the current network topology. If such a feedback between node state dynamics and dynamics of the network topology exists, the network is called adaptive network.

\subsubsection{Adaptive networks}

Adaptive networks have recently gained particular attention [62], as a variety of collective phenomena like swarming [63, 64], synchronization [65], selforganization to critical states $[66,67]$ or the emergence of complex topologies $[68,69]$ and distinct classes of nodes $[70,71]$ from an initially homogeneous population have been observed. A remarkable phenomenon is the appearance of different phases, in combination with phase transitions [72-74]. Reference [23] provides a comprehensive survey on adaptive networks.

As an example for an adaptive network model let us again consider the SISmodel introduced above, where we incorporate an additional process. The process takes into account that susceptible individuals try to avoid contact with 
infectious individuals and is defined in the following way:

$$
S I \stackrel{\omega}{\rightarrow} S S .
$$

This means, with probability $\omega$ a link from a susceptible to an infectious individual is broken. The susceptible individual then acquires a new link to a randomly chosen susceptible node from the network. It is intuitively clear that the additional rewiring rule in the adaptive SIS-model helps to contain an epidemic. Indeed, it was shown that the epidemic threshold increases, compared to the original SIS-model [72]. But, more interestingly, the dynamics of the adaptive $S I S$-model gives rise to a phase diagram where homogeneous regions (i.e. regions where either only healthy or only infectious individuals remain in the system), as well as regions of bistability and oscillatory behavior appear (see Fig. 2.3).

These rich dynamics, which are a common feature of adaptive networks, are mainly due to a competition between the dynamics of the network and the dynamics on the network. In Chapter 3 we discuss in detail an example where a contact process, which is coupled to a rewiring process leads to several emergent phases, separated by phase transitions. A central aim in this work is to determine the parameter regions where different phases occur, i.e. to calculate the phase boundaries. In the next section we provide the mathematical framework which is necessary for these calculations.

\subsection{Network analysis}

Capturing and analyzing network dynamics is one of the biggest challenges in the field of network science. Because of the typically huge amount of network constituents (nodes and links), their degrees of freedom give rise to an immensely high-dimensional state space. In order to make such a system mathematically tractable, one has to find methods to reduce the degrees of freedom, while keeping the relevant information. Having obtained a low-dimensional (approximate) description of the complex system, tools from dynamical systems theory can be applied to analyze the network dynamics.

In this section, we describe a common method to reduce the state space of a system consisting of many interacting subunits, which originates in statistical physics. Then, we introduce the basic concepts for analyzing the reduced system by means of dynamical systems theory.

\subsubsection{Reduction of the state space}

The complete information about the network at a certain time instant $t$ is captured in the microstate $n(t)$, which is defined by the adjacency matrix $\mathbf{A}(t)$ and the states of all nodes $\vec{\sigma}(t)$. Network dynamics then corresponds to a walk in the 
state space, spanned by all possible topological configurations and all possible distributions of node states. Tracking the precise position of the network at any time $t$ is in most cases not possible because of the high dimensionality of the state space and the stochasticity of the dynamical processes.

However, one can determine transition probabilities $t_{i j}$ between microstates $n_{i}$ and $n_{j}$. This leads to the so-called master equation,

$$
\frac{d}{d t} P\left(n_{i}, t\right)=\sum_{j} t_{j i} P\left(n_{j}, t\right)-t_{i j} P\left(n_{i}, t\right),
$$

which is a differential equation for the probability $P\left(n_{i}, t\right)$ to find the network in microstate $n_{i}$ at time $t$. Accordingly, $\sum_{i} P\left(n_{i}, t\right)=1$ holds at any time instant. Note that the master equation in (2.5) only applies to Markov processes, where the transition probabilities only depend on the current state, and not on the previous history of the dynamics. In many applications, Markov processes serve as reasonable descriptions of the actual dynamic processes.

In practice, it is in most of the cases not viable to determine all transition probabilities which enter in the master equation and then actually solve the equation. Typically, one considers therefore a reduction of the state space, which corresponds to a coarse graining. Such a reduction is in the first place, though a strong assumption, not a particularly restricting one. Usually, when considering dynamic networks, one is not interested in the state of every single node at a given time instant, i.e. in the microstate of the system, but rather in global quantities, i.e. the macrostate of the system. Such global quantities of interest are for example the number of nodes, or the number of links of a certain kind.

For example, in the SIS-model introduced above one is interested in the number of infected individuals, while their precise distribution, and the network topology are often irrelevant. This means, one can average over all irrelevant degrees of freedom, i.e. over all different microstates with the same number of infected individuals and thus reduce the dimensionality of the system drastically.

The described transition from microstates to macrostates is a principal concept in statistical mechanics. Formally, this form of coarse graining corresponds to a deterministic projection of the master equation, leading to average densities which can be described by deterministic equations [18]. These deterministic equations are valid in the "thermodynamic" limit, i.e. for infinitely large networks, but yield a good approximation already for sufficiently large finite systems. In order to capture the stochasticity of the process, one can, for example, manually add intrinsic or extrinsic noise to the coarse grained equations [75-77].

Passing from the master equation in (2.5) to evolution equations for average macroscopic quantities involves an additional practical aspect: The evolution equations for network motifs can often be directly derived from the dynamical 
rules of the system. Here, we introduced the general term motif, referring to a (usually small) subgraph of the network. The simplest motif is just one node. The ultimate goal is, to describe the network dynamics in terms of a set of differential equations for (appropriately chosen) motifs, or, more precisely, for motif densities.

The simplest set of motifs is given by the network moments, where the zeroth moment corresponds to the density of nodes, the first moment to the density of links, the second moment to the density of triplets, etc. The derivation of evolution equations for these moments, given a set of dynamical rules, is called moment expansion.

For illustration, we employ again the $S I S$-model introduced above and write down the evolution equation for the zeroth moment, the density of $I$-nodes [78]:

$$
\frac{d}{d t}[I]=-\gamma[I]+\beta[S I]
$$

Here, $[I]$ and $[S I]$ denote the densities of $I$-nodes and $S I$-links, i.e. their respective numbers divided by $N$. In words, equation (2.6) captures the loss of $I$-nodes due to recovery and the gain of $I$-nodes due to infection of susceptible neighbors, as described by the rules in (2.2). Despite the simple form of the equation, it is not straight-forward to solve it, because of the unknown link density $[S I]$.

Although we might proceed, deriving evolution equations for the first moments, the link densities, we will not arrive at a closed system of equations. The fact that the evolution equations for network moments depend on moments of higher order is inherent to a contact process and requires some sort of closure. Approximations which express higher order moments in terms of lower order ones are called moment closure approximations. The order of the approximation corresponds to the highest moment appearing in the equations. So, zeroth order equations only contain node densities, first order equations contain node densities and link densities, second order equations contain node densities, link densities and triplet densities, and so on.

Moment closure approximations are typically based on some kind of meanfield assumption. This means, they neglect heterogeneities and correlations beyond a certain order by assigning global averages to local variables. For example, in the simplest case the global connectivity $\langle k\rangle$ is assumed to hold for every single node in the network. In other words, all nodes are assumed to have mean degree. Consequently, every node is supposed to have in average $\langle k\rangle[I]$ neighboring $I$-nodes, irrespective of its own state. These assumptions lead directly to the zeroth order approximation, often simply called mean-field approximation, where $[S I]$ is approximated as $[S I] \approx[S][I]\langle k\rangle$ and (2.6) becomes

$$
\frac{d}{d t}[I]=-\gamma[I]+\beta\langle k\rangle[S][I] .
$$


Taking conservation of nodes into account, i.e. $[I]+[S]=1$, the equilibrium density of infected nodes yields

$$
[I]^{*}=1-\frac{\gamma}{\beta\langle k\rangle} .
$$

A comparison with (2.3) confirms a non-zero density of infected nodes beyond the epidemic threshold $\epsilon$. As mentioned earlier, this result is only valid for sufficiently homogeneous and uncorrelated networks. Otherwise, heterogeneous mean-field approaches can be used, where nodes are divided into classes according to their state and degree. Nodes belonging to the same class are then considered to be equivalent. This corresponds to an expansion of the motif basis $\{[I],[S]\}$ to a set of motifs of type $\left\{\left[I_{k}\right],\left[S_{k}\right]\right\}$, where $k$ is the degree. Such enhanced mean-field approaches have been successfully applied to scale-free networks $[48,49]$ and networks with correlated degrees [79, 80].

For analyzing the dynamics in adaptive networks, a zeroth order approximation is in principle not applicable, because in order to capture topological changes, at least the equations for the link densities have to be considered. The lowest possible order of closure is therefore a first order approximation, also referred to as pair-approximation. For the adaptive SIS-model the evolution equations for the link densities can be directly derived from the rules in $(2.2)$ and (2.4), yielding [78]

$$
\begin{aligned}
\frac{d}{d t}[S I] & =-(\gamma+\beta)[S I]+2 \gamma[I I]+\beta([S S I]-[I S I]), \\
\frac{d}{d t}[I I] & =\beta([S I]+[I S I])-2 \gamma[I I] .
\end{aligned}
$$

In the pair-approximation, triplet densities are expressed in terms of link densities in the following way [50, 78]:

$$
[A B C]= \begin{cases}\frac{[A B][B C]}{[B]} & \text { for } A \neq B \text { and } B \neq C, \\ \frac{2[A B][B C]}{[B]} & \text { for } A=B \text { or } B=C .\end{cases}
$$

Accounting for conservation of links, $[S I]+[I I]+[S S]=\langle k\rangle / 2$, and using the approximation scheme given in (2.9) the equations (2.6) and (2.8) constitute a closed set of differential equations for the zeroth and first network moments, which can be solved numerically [72].

Here, we presented a powerful approach to reduce the probabilistic, and in general not solvable master equation to a deterministic low-dimensional set of (non-linear) differential equations for network motifs. The crucial point in this procedure is to choose an appropriate motif set and to check the validity of the 
closure. The motif set and the approximations discussed here have been used in many applications, including adaptive networks [72, 81-85]. However, there are situations where this kind of approximation fails, because relevant heterogeneities and correlations are neglected. It has been shown that in these cases even closures of higher order or heterogeneous mean-field approximations do not significantly improve the predictions [83]. Therefore, it appears reasonable to consider a different motif set. This issue will be discussed in more detail in Chapter 3, where we develop an appropriate motif set for a class of models, which cannot be treated with a conventional moment closure approximation.

\subsubsection{Tools from dynamical systems theory}

In the last section, we sketched a method for the derivation of a low-dimensional system of deterministic differential equations, which describes the dynamics of macroscopic network quantities. Having obtained such a (in general non-linear) system of differential equations,

$$
\frac{d}{d t} \vec{x}(t)=\vec{F}\left(\vec{x}(t),\left\{p_{i}\right\}\right)
$$

for the motif densities $\vec{x}$ and a set of parameters $\left\{p_{i}\right\}$, further investigation of this system requires some concepts from dynamical systems theory. So, in the following we briefly introduce the basic ideas and tools from the theory of dynamical systems which we will employ in the course of this work. For further reading we refer to common textbooks as e.g. [25, 86].

First of all, we note that (2.10) is an autonomous system of differential equations, i.e. the temporal evolution of the variables does not explicitly depend on time. Solutions $\vec{x}(t)$ of (2.10) for a specified vector field $\vec{F}$ and given parameters $\left\{p_{i}\right\}$ are called trajectories in the state space. Depending on the systems complexity, individual trajectories can be computed analytically or numerically. However, the focus in this work is less on the temporal evolution of a dynamical system, but rather on the steady states, and in particular on the nature of the steady states.

A steady state or fixed point $\vec{x}^{*}(t)$ is a special trajectory, namely a point in the state space corresponding to a constant solution of the dynamical system. This implies $\vec{F}\left(\vec{x}^{*}, \vec{p}\right)=0$. The nature of the fixed point determines the behavior of trajectories close to the fixed point. More precisely, if a trajectory starting close to the fixed point stays close, the fixed point is said to be stable, otherwise, if a trajectory starting close to the fixed point moves away, the fixed point is said to be unstable. If a trajectory, starting in the neighborhood of a fixed point reaches the fixed point for $t \rightarrow \infty$, the fixed point is said to be asymptotically stable. A major concern in analyzing systems of type (2.10) in the context of network theory is to find the fixed points and determine their stability. 
For example in the SIS-model, a fixed point of $(2.7)$ is clearly $[I]^{*}=0$, the global healthy state. If this fixed point is stable, a small perturbation of this state, i.e. a small number of infected individuals does not lead to an epidemic outbreak, but the global healthy state is recovered. If the fixed point is unstable, a small number of infected individuals propagates the disease and causes further infections, driving the system away from the fixed point.

A mathematical framework to determine the stability of a fixed point is linear stability analysis. It is based on a first order Taylor expansion of $\vec{F}\left(\vec{x}(t),\left\{p_{i}\right\}\right)$ in the fixed point $\vec{x}^{*}$, leading to a linearized system

$$
\frac{d}{d t} \vec{y}(t)=\mathbf{J}\left(\vec{x}^{*}\right) \vec{y}(t)
$$

which is locally qualitatively equivalent ${ }^{3}$ to the original system in (2.10). The Jacobian matrix $\mathbf{J}$ is defined as $J_{i j}=\partial F_{i} / \partial x_{j}$. For the linear system in (2.11), the stability of the fixed point $\vec{y}^{*}=0$ is determined via the eigenvalue spectrum of $\mathbf{J}$. More precisely, if all eigenvalues of $\mathbf{J}$ have negative real parts, $\vec{x}^{*}$ is stable, otherwise $\vec{x}^{*}$ is unstable. In practice, one often uses the maximal real part of all eigenvalues, $\lambda(\mathbf{J})$, as an indicator for stability. This leads to the following classification of fixed points:

$$
\lambda(\mathbf{J}) \quad\left\{\begin{array}{rll}
<0 & \Rightarrow & \text { stable } \\
>0 & \Rightarrow & \text { unstable. }
\end{array}\right.
$$

In this work, when we deal with purely real eigenvalue spectra, we refer to $\lambda(\mathbf{J})$ simply as the "largest eigenvalue" of $\mathbf{J}$.

So far, we ignored the parameters $\left\{p_{i}\right\}$ that appear in the model and therefore in the derived dynamical equations. But we have to consider that the nature of the fixed points depends on these parameters. So, for example, a stable fixed point can turn into an unstable one or vice versa if one or more of the parameter values are altered.

Recalling again as an example the SIS-model, we find that the Jacobian matrix at the fixed point $[I]^{*}=0$ is given by $\mathbf{J}(0)=-\gamma+\beta\langle k\rangle$, when we consider the mean-field approximation given in (2.7). In this case, the Jacobian matrix is just a number and we obtain with (2.12) that the global healthy state is stable if $\beta / \gamma<1 /\langle k\rangle$. This example shows that the fixed point is only stable under certain conditions on the parameters, whereas at some critical parameter setting, it becomes unstable. In the SIS-model the critical parameter setting is called epidemic threshold (compare (2.3)).

\footnotetext{
${ }^{3}$ Two dynamical systems are qualitatively equivalent if the number, the ordering and the nature of their fixed points are the same. The equivalence to the corresponding linearized system only applies if all eigenvalues of $\mathbf{J}$ have non-zero real parts [25].
} 
In general, the critical parameter setting $\left\{p_{i}^{c}\right\}$ where a fixed point changes its nature is called a bifurcation. Bifurcation theory comprises a variety of different types of bifurcations [86]. Here, we are mainly concerned with local bifurcations, and among local bifurcations with those bifurcations where a fixed point changes stability $^{4}$. Due to (2.12), a fixed point for example looses stability if the real part of the largest eigenvalue of the corresponding Jacobian matrix becomes positive. The physical manifestation of a bifurcation is a phase transition. The critical parameter settings define points, lines, or higher dimensional manifolds, which separate two distinct phases. In our example, the line $\epsilon=1 /\langle k\rangle$ separates a healthy phase from an endemic phase.

Now, we have come to the point where we have all the prerequisites to compute phase transitions in network models. The remainder of this work is dedicated to refinements of these principal concepts and to applications for different classes of network models. In the considered models we particularly focus on phase transitions between phases of high diversity and phases of low diversity.

\footnotetext{
${ }^{4}$ In our applications we are not interested in those fixed points which possibly appear or vanish in a bifurcation, but only in the fixed point that changes stability.
} 


\section{Fragmentation transitions in models for opinion formation}

A flourishing field of applications for network science is the complex dynamics of social systems [87-90]. Related investigations comprise both, the analysis of data available from online social networks or from mobile phone calls [9197 , and the analytical and computational modeling of dynamical processes in networked societies $[57,98,99]$. Concerning both approaches, studying opinion dynamics has currently attracted particular attention [57, 100-103].

Typically, in models for opinion formation a society is described as a network, where nodes correspond to individuals and links correspond to social relationships. The opinion of an individual is captured by the internal state of a node, which changes in time due to interactions with the social environment. The pattern of interactions, which is described by the network topology, in turn evolves according to the internal states of the nodes. So, this type of opinion formation process, where the opinion dynamics on the network and the dynamics of the network topology are coupled, can be adequately captured by an adaptive network [23, 62].

Depending on the specific model, the node states can be discrete, resembling a voter situation, where individuals choose one candidate or one party from a given set of options $[73,100,104]$. The node states can alternatively be treated as continuous variables, representing e.g. the strength of a belief or the inclination to some opinion [105-108], or the node states assume vectors, taking into account a whole set of individual traits $[109,110]$.

The interactions between individuals can be implemented in many different variants $[29,82,101,111-113]$. However, usually there are two main processes governing the dynamics of the system: social adjustment and social segregation. Social adjustment accounts for the tendency of individuals to copy the opinion of their closest acquaintances, while social segregation captures the tendency of individuals to preferably interact with like-minded people. As both processes aim to reduce conflicts and convey homogeneity, in the absence of external influences like media and spontaneous intrinsic opinion changes, the dynamics stops as soon as there are no individuals with different opinions connected to each other. This can be the case, either when the whole system reaches a homogeneous state, i.e. everyone holds the same opinion, or when the system breaks into disconnected groups of different opinions, which are internally homogeneous. The first outcome is referred to as consensus, while the latter represents a fragmentation of the system.

Whether consensus or fragmentation is reached, depends on the balance between adaptation and segregation, or more precisely on the relative rates at which both processes occur. In the limit where adaptation is much faster than 
segregation consensus is reached, whereas if segregation is much faster than adaptation, fragmentation occurs. The phase transition in parameterspace from a consensus to a fragmented state is called fragmentation transition. Fragmentation transitions have been frequently observed in simulations [73, 100, 101, 105]. However, common analytical approaches that faithfully capture other phase transitions $[72,81,84]$ yield only rough approximations for the fragmentation threshold $[114,115]$. In this chapter we present a novel approach, which allows for a precise analytical estimation of fragmentation thresholds. A major part of the described methods and applications was published in [115-118].

In Section 3.1 we introduce the adaptive voter model as a simple and paradigmatic example for the discussion of fragmentation transitions. Then, in Section 3.2 , we propose an analytical approach for the calculation of fragmentation transitions and apply it to the adaptive voter model. We extend the analysis of fragmentations to voter models with an arbitrary number of states in Section 3.3 and present further applications of our analytical approach in Section 3.4. Section 3.5 provides a summary and discussion of the present chapter.

\subsection{The adaptive voter model}

The adaptive voter model was introduced as a paradigmatic model for opinion formation in networked populations $[29,73,114,119]$. It is based on the original voter model [120], which describes a network where nodes represent agents and links represent social contacts. Each agent can hold either of two opinions, say $A$ and $B$. Then, the opinions are updated by randomly choosing two neighboring nodes and changing the opinion of one of the nodes. Because in the original model the topology remains fixed, the dynamics continues until global consensus is reached.

Adaptive variants of the voter model take an additional process into account, the rewiring of links. Rewiring leaves the number of agents and links unchanged but alters the structure of the network. Specifically, it can cause a fragmentation of the network, such that both opinions survive in disconnected network components which are internally in consensus.

\subsubsection{Definition of the model}

There exist many different implementations of the adaptive voter model, concerning the update rule $[29,115,119]$ and the type of rewiring $[83,100,111,112]$. Here, we discuss an adaptive voter model with the following update mechanism (see Fig. 3.1): in every time step one random link is chosen. If it is an inert link, i.e. a link which connects nodes in equal states ( $A A$ - or $B B$-link), nothing happens. If the chosen link is an active link, i.e. a link which connects nodes in different states $(A B$-link), then with probability $p$ a rewiring event occurs 


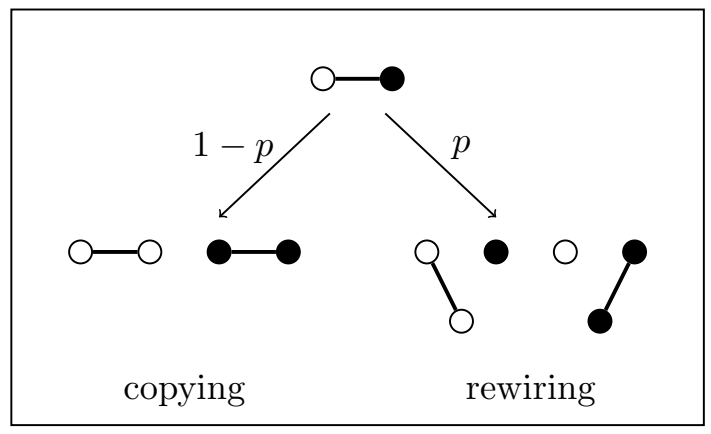

Figure 3.1: Update rule for the adaptive voter model (link update). On a randomly chosen active link with probability $1-p$ a copying event occurs, whereas with probability $p$ a rewiring event occurs. In a copying event, one of the nodes is set to the neighbor's state. In a rewiring event, the original link is removed and replaced by a new link between one of the two nodes and a randomly chosen node in the same state. Both, copying and rewiring, occurs with equal probability to either of the nodes from the selected pair.

and with probability $1-p$ an opinion update occurs. In a rewiring event the $A B$-link is removed and a new link is established between either the $B$-node and a randomly chosen node of state $B$, or between the $A$-node and a randomly chosen node of state $A$. In an opinion update, one of the connected agents copies the other's state, i.e. either the $B$-node becomes an $A$-node or vice versa. In this so-called link update rule, it is decided randomly and with equal probability which one of the chosen pair of nodes adopts the opposite opinion or rewires a link.

The adaptive voter model is characterized by two different dynamical regimes, as a consequence of the competing timescales which govern adoption and rewiring processes $[73,114]$. As both processes are coupled by one single parameter, the rewiring rate $p$ determines the long-term behavior of the system: 1) For relatively small values of $p$ the system reaches consensus, i.e. basically all nodes assume the same state. Note that in this regime, the system first reaches an active steady-state, where both types of nodes are still present. Only eventually finite size fluctuations lead to the disappearance of one type of nodes. 2) For values of $p$ beyond a critical value $p_{c}$, the system fragments into two disconnected clusters of opposite states. One challenging task is to predict the fragmentation threshold $p_{c}$, where the phase transition occurs. 


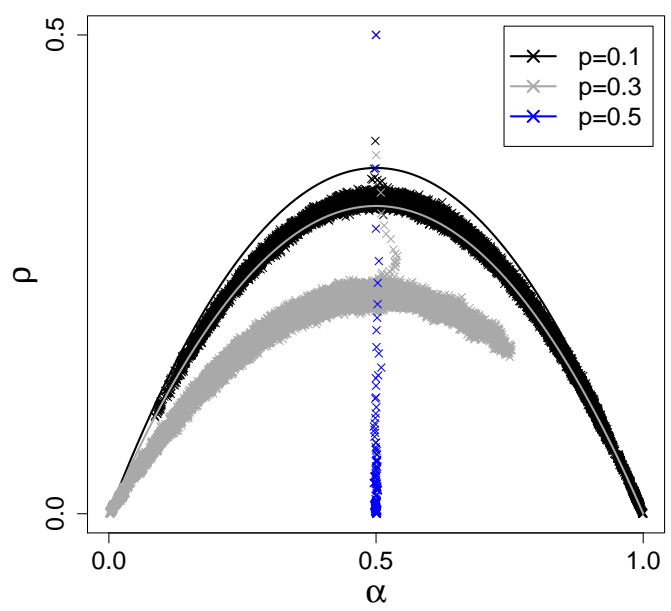

Figure 3.2: Steady states in the adaptive voter model. For $p=0.1$ and $p=0.3$ the system performs a random walk on a parabola of steady states until one of the absorbing consensus states is reached. For $p=0.5$ the parabola of steady states does not exist and the system rapidly reaches a fragmented state. Lines correspond to the expression given in (3.1), obtained from a pair-approximation. It becomes clear that the conventional approach is only valid for $p \ll p_{c}$. N $=$ $10^{4},\langle k\rangle=4, \alpha=0.5$.

\subsubsection{Conventional calculation of the fragmentation transition}

One way to analyze the fragmentation transition in the adaptive voter model is by means of a conventional moment expansion [73, 83, 114, 115]. As described in Section 2.3.1, in this approach network moments are defined as node densities, link densities, triplet densities, etc. Then, evolution equations for these moments are formulated and evaluated at the steady state. Typically, the evolution equation for one specific moment involves higher order moments. Therefore, one has to break up the expansion at a certain order and close the system of equations. This is called a moment closure approximation [50].

Using a first order moment closure approximation, a pair-approximation, one can calculate the equilibrium value of the active link density $[83,112,115]$ :

$$
\rho=\frac{2(\langle k\rangle(1-p)-1)}{\langle k\rangle(1-p)} \alpha(1-\alpha) .
$$

Here, $\rho$ denotes the active link density, i.e. the number of $A B$-links devided by 
the total number of links and $\alpha$ denotes the fraction of $A$-nodes. Equation (3.1) corresponds to a parabola in the $\rho$ - $\alpha$-plane (see Fig. 3.2). In the limit of infinite system size, fluctuations vanish [73], which implies that the initial fraction of $A$-nodes, and consequently the initial fraction of $B$-nodes, is conserved. This means that in the thermodynamic limit every point on the curve described by (3.1) corresponds to an attractor for a certain initial distribution of node states. In finite systems, the numbers of nodes in state $A$ and $B$ fluctuate, and accordingly the active link density varies. In fact, the system performs a random walk in the $\rho$ - $\alpha$-plane, constrained by the shape of the parabola, until it reaches one of the absorbing endpoints $\alpha=0$ or $\alpha=1$. Once an absorbing state is reached, the dynamics stops, as either all nodes are in state $A$ or all nodes are in state $B$ and there are no active links remaining. The described behavior corresponds to regime 1) where (eventually) consensus is reached. Note that the time to actually reach consensus can take immensely long in large systems [29]. We therefore often refer to this regime as an active phase, because it is characterized by a finite density of active links during a relatively long time period.

On the other hand, from (3.1) follows that at $p_{c}=(\langle k\rangle-1) /\langle k\rangle$ the parabola of steady states vanishes. So, for $p>p_{c}$ only the line $\rho=0$ is attractive and the system quickly reaches an absorbing state on that line (see Fig. 3.2). For example, if we start with equal distribution of states, an absorbing state at $\alpha \approx 1 / 2$ is reached. This means that half of the nodes is in state $A$, half of the nodes is in state $B$ and there are no links connecting nodes in different states. This corresponds to regime 2), the fragmented state.

In Fig. 3.2 we plot the active link density for different values of $p$, comparing simulation results and the analytical approximation given in (3.1). One can clearly see the parabolic shape of the active link density for $p$-values below the critical point, as predicted by (3.1), and a rapidly decaying active link density for $p$ above the critical point. However, it also becomes clear that the precision of the pair-approximation decreases close to the fragmentation point. In particular, the critical point itself is significantly overestimated: for $\langle k\rangle=4$ simulations yield $p_{c} \approx 0.46$, while the pair-approximation predicts $p_{c}=3 / 4$.

The reason for the failure of the moment closure approach in predicting the fragmentation threshold is the following $[115,121]$ : Close to the fragmentation point, the remaining active links are not homogeneously distributed all over the network, as it is assumed in the mean-field-like approximation. In fact, they are rather concentrated in a small number of bundles connecting the almost fragmented clusters of opposite states (see Fig. 3.3). In the following section we introduce an analytical approach which accounts for this specific situation by choosing an appropriate basis for the moment expansion. 


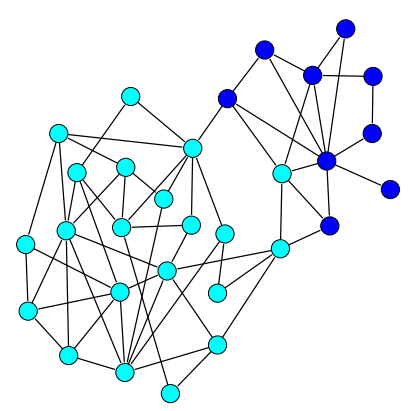

Figure 3.3: Network configuration close to the fragmentation point. For illustration, we show a network with a small number of nodes. Active links which connect the two components of opposite states are scarce but concentrated at few nodes (active motifs). Such a situation is the starting point for the percolation approach.

\subsection{Percolation approach for the calculation of fragmentation thresholds}

In this section, we present an alternative approach, which allows for a precise estimation of fragmentation thresholds. A major part of this section was published in [116]. We use the adaptive voter model introduced in Section 3.1.1 as an example to explicitly demonstrate the procedure of our approach and compare the results to agent-based simulations. However, we emphasize that the approach is applicable to a wide range of models employing various update mechanisms.

The approach we propose here is inspired by the concept of percolation. Percolation theory was originally applied to study flows in porous media [122]. There, one is interested in a situation, where the fluid traverses (percolates) the porous media. Because of the parallels to cluster formation in networks, percolation theory has proven a powerful concept in network science, both for the characterization of graph topologies (e.g. in the context of giant component formation [123-125]) and for the characterization of the dynamics taking place on networks, like invasion and spreading processes [126-128]. Nevertheless, in adaptive network models, where the dynamics affects the topology and the node states, percolation approaches were barely used so far. 
Typically, models for disease spreading make use of the percolation idea for the computation of epidemic thresholds [129-131]. In these models, one considers a network of susceptible agents and determines the probability for an epidemic outbreak, i.e. an infection of a finite fraction of the population, starting from a single infected individual. Here, the idea is to study percolation of active links. This means, we determine the probability for a single active link to propagate in an inert environment, i.e. in a cluster of inert links. Therefore, we consider a situation close to the fragmentation point, where the network is composed of two almost disconnected homogeneous clusters of opposite opinions, which are joined by only few active links ${ }^{5}$ (see Fig. 3.3). Then, we determine the probability for these active links to spread within the homogeneous compartments.

In order to account for multiple active links connecting to the same node we introduce a basis of active motifs for the derivation of the evolution equations. In the following, we present two possible motif bases: a simple one which allows for a quick and reasonable estimation of the fragmentation point and a refined variant which yields predictions with high accuracy. Using these basis sets, we derive dynamical equations capturing the net change in the density of active motifs. If this change is negative then the number of active links declines exponentially leading eventually to the fragmented state. If the balance is positive then active links proliferate and prevent the network from reaching the fragmented state. The critical point, where the fragmentation transition occurs corresponds to a vanishing net change in the density of active motifs.

\subsubsection{Basis of $q$-fans}

First, let us consider the simplest set of active motifs which captures the essential features relevant for the prediction of the fragmentation transition. This basis is a set of $q$-fans. A $q$-fan is defined as a subgraph consisting of one node holding a given opinion and $q$ neighbors of the node holding the opposite opinion. The node holding the solitary opinion is denoted as the base node of the fan, whereas the other nodes are denoted as the fringe nodes. For instance a 4-fan contains a base node and four fringe nodes, which are connected to the base node via active links. Note that the base node has at least one inert link, leading to the neighbor which caused the opinion change (see Fig. 3.3). For the $q$-fan basis we assume that the base node has exactly one inert link. Later we relax this assumption.

For simplicity, let us first assume that the network is degree-regular, meaning that every node has exactly the same number of neighbors, $k=\langle k\rangle$. The dynamics of active motifs for the special case of $k=3$ is illustrated in Fig. 3.4.

\footnotetext{
${ }^{5} \mathrm{By}$ few active links we mean that the number of active links is small compared to the total number of links. One can interpret this situation as a perturbation of the fragmented state.
} 


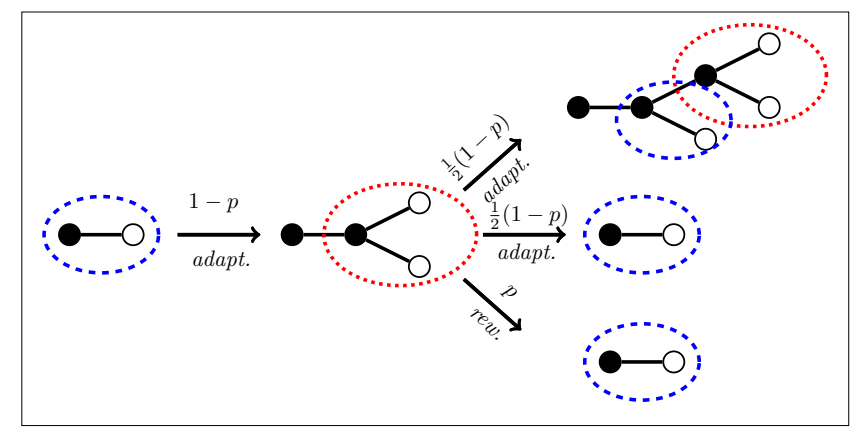

Figure 3.4: Illustration of the evolution of $q$-fans in a degree-regular network with degree $k=3$. Agents are depicted as nodes which are open or solid depending on their opinion. Shown is the network in the neighborhood of an active link connecting two clusters of different opinions. Arrows correspond to adaptation and rewiring events and are labeled with the corresponding transition rates. Depending on the parameters, the updates lead to proliferation or decline of isolated active links (encircled dashed) and 2-fan motifs (encircled dotted).

We start from a single active link (left half of figure) connecting two almost fragmented clusters of opposite opinions. In an update event, with probability $p$ the active link is rewired becoming inert (not shown) or, with probability $1-p$, one of the nodes adopts the other's opinion. In the adoption event the original active link becomes inert, but the two other links of the adopting agent become active, giving rise to a 2 -fan. We continue by studying how updates affect this 2 -fan (Fig. 3.4, right half). If an update is a rewiring event (probability $p$ ) then it decreases the width of the fan, turning the 2-fan into a single active link. If the update is an opinion adoption event (probability $1-p$ ) then there are two possible scenarios occurring with equal probability. In the first scenario the node at the base of the fan changes its opinion. In this case the 2 -fan becomes inert, but one new active link is formed at the base of the fan. In the second scenario one of the fringe nodes of the fan adopts the base node's opinion, in this case the width of the fan is reduced by one, but an additional 2-fan is activated. Because the two active motifs in the latter scenario are now separated by an inert link they can be assumed to be independent to good approximation.

The transitions for the $q$-fans give rise to a $k$-1-dimensional system of differential equations (see Fig. 3.5). For the example of degree-regular networks with $k=3$ we obtain the following two-dimensional system,

$$
\begin{aligned}
& \{\dot{1}\}=-\{1\}+\{2\}, \\
& \{\dot{2}\}=-\{2\}+(1-p)\{1\}+\frac{1}{2}(1-p)\{2\},
\end{aligned}
$$


where $\{q\}$ is a rescaled $q$-fan density, referring to the number of $q$-fans multiplied by the number of active links in the fan, $q$, and normalized by the total number of links $L$.

As the systems of equations obtained by this approximation are linear, the Jacobian matrix is just the coefficient matrix. In general, for non-linear systems the stability of the fragmented state can be tested by a local linearization, where the Jacobian matrix is given by $J_{i j}=\partial\{\dot{i}\} / \partial\{j\}$ (see Section 2.3.2). Here, for $k=3$,

$$
\mathbf{J}=\left(\begin{array}{cc}
-1 & 1 \\
1-p & -1+\frac{1}{2}(1-p)
\end{array}\right)
$$

The fragmented state, $\{i\}=0(i=1,2)$, is stable if all eigenvalues of the Jacobian matrix have negative real parts. For linear systems this state is then also globally attractive. The fragmentation transition occurs in the bifurcation where eigenvalues cross the imaginary axis. For $k=3$ the transition point can be calculated from (3.3) and yields $p_{c}=1 / 3$.

We note that the stability of global consensus states, which are also characterized by $\{i\}=0$ is not captured by the same Jacobian because these states violate our assumption of the presence of two almost disconnected clusters. Although adaptations for other transitions may be possible, the method, as proposed here, only captures transitions to the fragmented state.

Degree-regular networks with $k>3$ can be treated analogously to the $k=3$ example. The corresponding equations for $k=4$ and the generalization to arbitrary $k$ are shown in Fig. 3.5. An update affecting an active link deactivates the link and activates a $k-1$-fan with probability $1-p$. An update affecting a $q$-fan either a) deactivates the fan and activates a single link (probability $(1-p) / 2) ; \mathrm{b})$ decreases the width of the fan by one, turning the $q$-fan into a $q$-1-fan (probability $p$ ); or c) decreases the width of the fan by one and activates a new $k-1$-fan (probability $(1-p) / 2$ ).

As it can be seen in Fig. 3.6, already this simple motif set yields a more precise estimate of the fragmentation point than the pair-approximation. In the following, we introduce an enhanced basis which also accounts for the number of inert links. Moreover, we allow for in principle arbitrary degree distributions, as degree-regularity, even if present in the initial network, is destroyed by the rewiring process. 


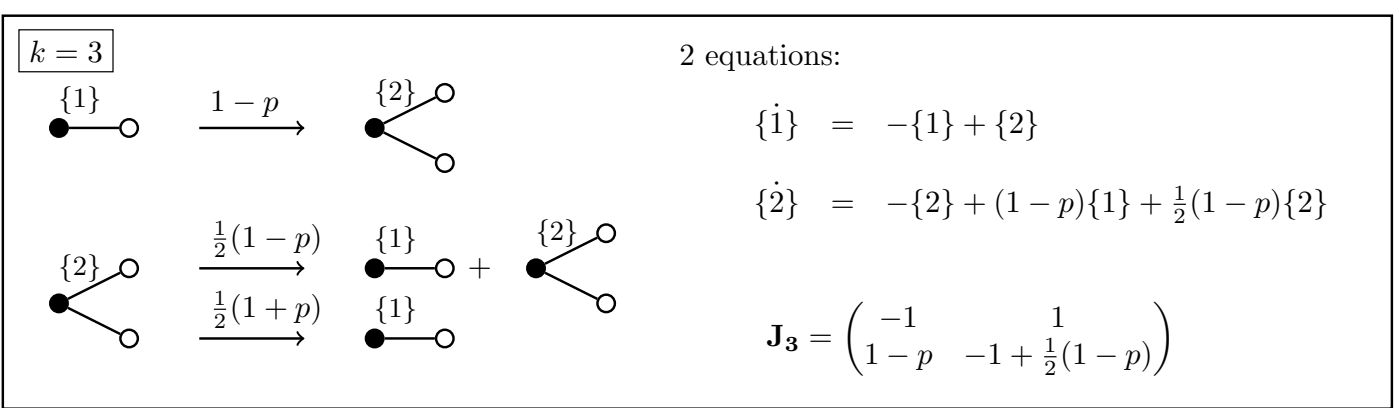

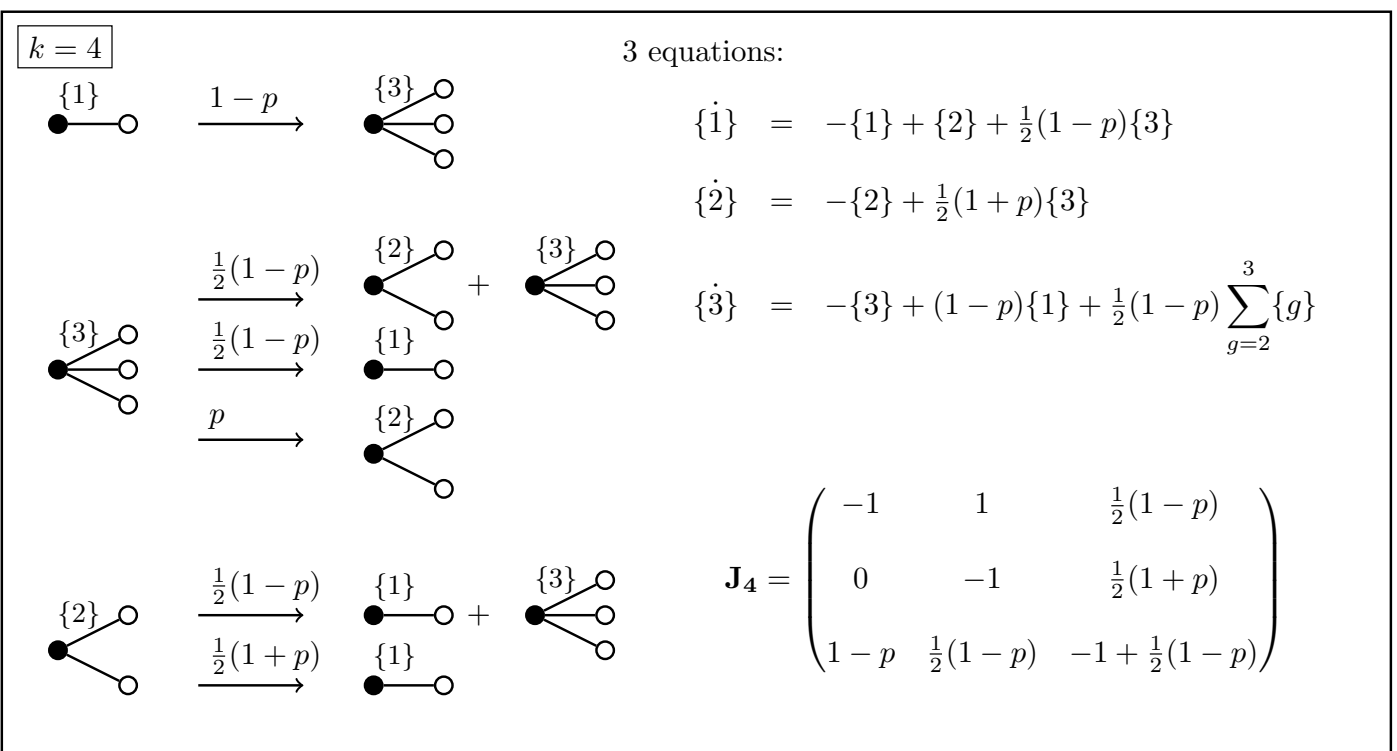

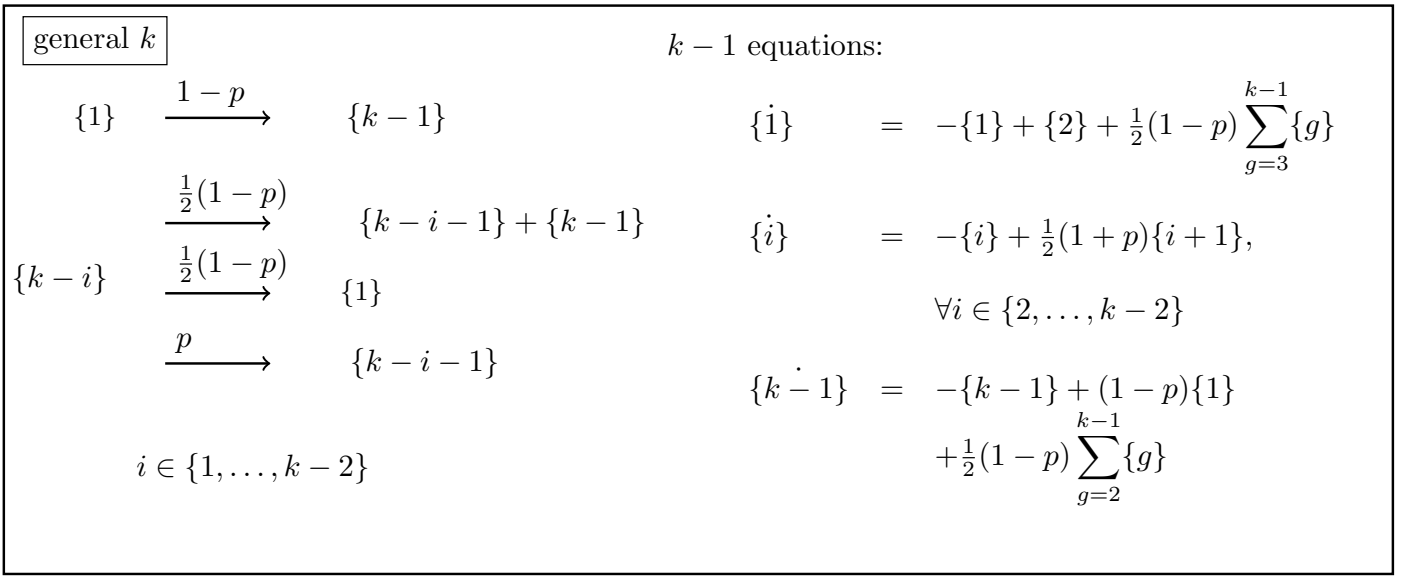

Figure 3.5: Illustration of the transitions for $q$-fans in degree-regular networks with degree $k$. Motifs and transitions are depicted as in Fig. 3.4. Additionally, the corresponding systems of differential equations and the Jacobian matrices are shown. 


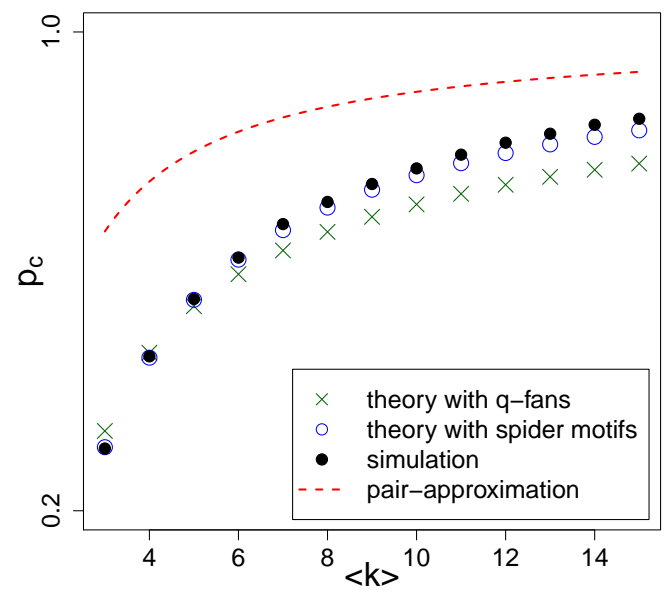

Figure 3.6: Fragmentation threshold in the adaptive voter model. Shown are numerical results from agent based simulation $(\bullet)$, pair-approximation (dashed line), the analytical approach proposed here $(\times)$, and its refined variant $(\circ)$. The proposed approaches yield a better match with the numerical results than the established procedure. $N=10^{6}$.

\subsubsection{Basis of spider motifs}

In order to improve the prediction for the fragmentation threshold we introduce spider motifs. Spider motifs consist of one central base node connecting to $m$ nodes of its own opinion and $l$ nodes of the opposing opinion. The $\{m, l\}$-spider thus holds $m$ inert links and $l$ active links, leading to a total degree $k=m+l$. As before, we do not account for all motifs in the network but consider only active spiders, i.e. $l>0$.

The effects of updates on a spider motif are shown schematically in Fig. 3.7. In a rewiring event either the rewired link is kept by the fringe node and the $\{m, l\}$-spider is turned into a $\{m, l-1\}$-spider or the rewired link is kept by the base node turning the $\{m, l\}$-spider into a $\{m+1, l-1\}$-spider. In an opinion adoption event either the base node is convinced, which turns all active links into inert links and vice versa, leading to a $\{l, m\}$-spider, or one of the fringe nodes is convinced by the base node giving rise to a new $\{1, g\}$-spider while in the focal spider one active link turns into an inert link. The evolution of spider 


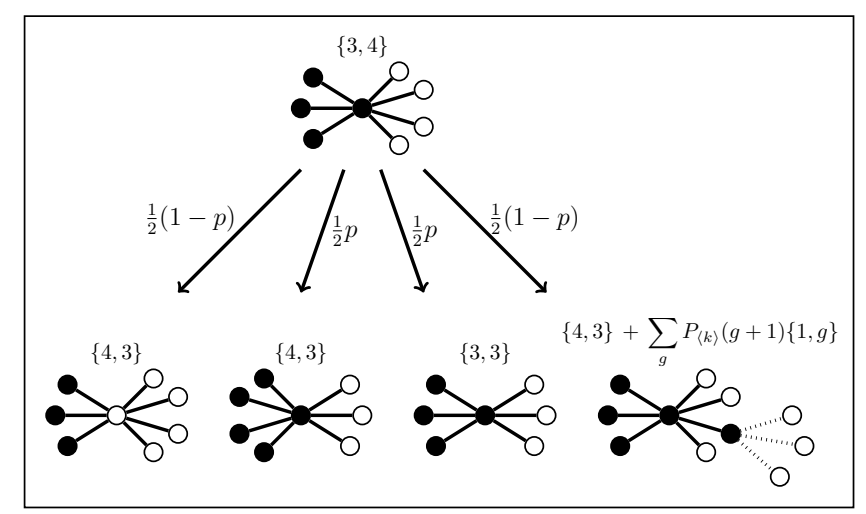

Figure 3.7: Transition probabilities for a $\{3,4\}$-spider. Diagrams and arrows represent motifs and transitions in analogy to Fig. 3.4. The dashed links indicate the number of next-nearest neighbors, which is drawn from the degree distribution (here, Poissonian distribution).

densities thus follows

$$
\{m, l\} \begin{cases}\stackrel{\frac{1}{2}(1-p)}{\longrightarrow}\{l, m\} \\ \stackrel{\frac{1}{2}(1-p)}{\longrightarrow}\{m+1, l-1\}+\sum_{g=1}^{k_{\max }-1} P(g+1)\{1, g\} \\ \stackrel{\frac{1}{2} p}{\longrightarrow} & \{m+1, l-1\} \\ \stackrel{\frac{1}{2} p}{\longrightarrow} & \{m, l-1\},\end{cases}
$$

where the degree of the activated spider is drawn from the degree distribution ${ }^{6}$. In an adaptive network the degree distribution is often unknown because it is reshaped by the rewiring events. However, the success of the degree-regular approximation suggests that good results can be obtained if reasonable distributions are used. The results in Fig. 3.6 were obtained by assuming a Poissonian degree distribution $P(k)=e^{-\langle k\rangle}\langle k\rangle^{k} /\langle k\rangle$. Further, we considered only spiders up to a maximal degree of $k_{\max }=50$. We constructed the Jacobian matrix by computer algebra and determined the fragmentation point from numerical computation of eigenvalues. Fig. 3.6 shows that the results from the refined procedure are in good agreement with numerical simulations.

The small discrepancy between our theoretical estimation and simulation results for high mean degrees (Fig. 3.6) might stem from the approximations

\footnotetext{
${ }^{6}$ Note that, as the focal node of the new spider can be reached only via one possible link, this situation is not the same as following a random link.
} 
involved in our analytical approach. There are basically three approximations, still present in the refined variant: 1) We do not account for additional inert links due to rewiring. 2) We assume independence of a new active motif from the originating motif. 3) We neglect cycles.

Regarding approximation 1), let us recall that we consider the limit of vanishing spiders, where it is very unlikely for the target node in a rewiring event to coincide with the focal node of a spider. Moreover, as our simple basis set (which does not account for the number of inert links at all) already yields good results, the rare event of a spider gaining an inert link is not likely to have a significant effect on the estimated fragmentation point.

Approximation 2) implies that a process where a new spider is created (last transition in Fig. 3.7) is irreversible. This means, the remainder of the originating spider and the new spider are considered to be independent and there is no process which simultaneously deactivates the new spider and recovers the originating spider in its previous form. Although it is hard to estimate the magnitude of the introduced error, one can argue that approximation 2) should lead to an overestimation of the fragmentation point. What we see in Fig. 3.7 is an underestimation of the fragmentation point for high mean degrees. So, we can argue that if approximation 2) has an effect on the result, there must be an opposing effect which is stronger.

Finally, let us consider approximation 3), which neglects any links between fringe nodes. While in a random graph clustering is negligible for $N \rightarrow \infty$ [132], it turns out that the rewiring rule implemented in the adaptive voter model facilitates the formation of triangles. Simulations show that the clustering coefficient increases for $p \rightarrow p_{c}$. As the clustering coefficient also increases with the mean degree, we conclude that ignoring cycles presumably yields the strongest assumption in our approach and therefore might be responsible for the minor discrepancies in our analytical results.

Let us summarize the main content of this section. Here, we presented an approach for the analytical estimation of fragmentation thresholds which is based on the idea of percolation. Starting from a configuration close to the fragmentation threshold we derive evolution equations for active motifs. Demanding a positive net balance of active links then yields a condition from which we can extract the fragmentation threshold. The crucial point in this procedure is to find a suitable basis of motifs which comprises the main feature of the system close to the fragmentation point. The motif sets we propose account for the clustering of active links which is observed close to the fragmentation point, implying simultaneous creation of several active links which are connected to the same node. Even in the limit of low average active link density, such links cannot be treated independently because they all become inert at once if the focal node changes its opinion. Mean-field-like approximations (like moment closure approximations) ignore these correlations by assuming a well-mixed system. This 
might explain their poor estimate for the fragmentation threshold. Using the example of an adaptive voter model we showed that the results obtained from the approach described here match the fragmentation points observed in simulations very well. In the following sections we present further examples for the applicability of the described approach.

\subsection{Fragmentation transitions in multi-state voter models}

In this section, we study fragmentation transitions in more realistic voter models, using the analytical approach described in the previous section. The work presented here was published in [118].

While most of the voter-like models only consider a binary choice of opinions, many real-world situations offer a large number of choices. In the physics literature some models for opinion formation, which consider arbitrary-many opinions have been studied $[83,100,111]$. In these models all opinions are "equidistant" in the sense that all interactions between any given pair of (different) opinions follow the same dynamical rules. Models recognizing that the outcome of interactions may depend on a measure of similarity (or distance) between opinions are often considering an uncountable set of opinions and are therefore hard to treat analytically $[105,106]$.

In the following, we consider a natural extension of the original adaptive voter model, where we allow for an arbitrary countable set of opinions. In the proposed model the rewiring rate that governs the interaction of conflicting agents is assumed to depend on the specific pairing of opinions held by the agents. The model can thus account for heterogeneous "distances" between opinions. A large distance, characterized by a high rewiring probability, indicates a controversial pairing, whereas a small distance, and correspondingly low rewiring probability, indicates that the respective opinions are almost in agreement.

The assumption of heterogeneity in the space of opinions gives rise to a complete, weighted graph representing the relationships between different opinions. We will refer to this graph as the network of states. In contrast, the network of individuals is an unweighted graph with a given degree distribution, which represents the interactions between individuals. Fragmentations in this system occur naturally at the level of individuals, namely when several groups of individuals holding different opinions, separate from each other. However, for the analysis of fragmentations it is convenient to consider the state-network and associate a fragmentation in the network of individuals with a loss of links in the state-network. 


\subsubsection{Definition of the multi-state voter model}

We define the multi-state voter model as a natural extension of the adaptive voter model with link update, introduced in Section 3.1.1. Each node $\alpha$ holds a state $s_{\alpha}$, indicating its opinion. Initially node states are drawn randomly and with equal probability from the set of all states $\Gamma=\left\{g_{1}, g_{2}, g_{3}, \ldots, g_{G}\right\}$, where the total number of states is $|\Gamma|=G$ and we assume $G \ll N$, i.e. the number of states is much smaller than the number of individuals. Then, the system is updated as follows: In each update step a random link $(\alpha, \beta)$ is chosen. If $s_{\alpha}=s_{\beta}$, nothing happens. If $s_{\alpha} \neq s_{\beta}$, an update occurs on the link. A given update is either a rewiring event or an opinion adoption event, depending on the similarity of the respective opinions. For individuals $\alpha, \beta$ with opinions $s_{\alpha}=g_{i}$ and $s_{\beta}=g_{j}$, the update is a rewiring event with probability $p_{i j}$ and an opinion adoption event otherwise (probability $1-p_{i j}$ ). Following the conventions for the adaptive 2 -state voter model, the parameters $p_{i j}$ are called rewiring rates.

In a rewiring event, the focal link $(\alpha, \beta)$ is severed, and a new link is created either from $\alpha$ to a randomly chosen node $\gamma$ with $s_{\gamma}=s_{\alpha}$, or from $\beta$ to a randomly chosen node $\gamma$ with $s_{\gamma}=s_{\beta}$. The choice between the two outcomes is made randomly with equal probability. In an opinion update, either node $\alpha$ changes its state to $s_{\beta}$ or node $\beta$ changes its state to $s_{\alpha}$, where the choice between both outcomes is again made randomly with equal probability. In the following, we assume symmetric interactions, which implies $p_{i j}=p_{j i}$ such that the specific model is characterized by a set $\left\{p_{i j}\right\}$ of $G(G-1) / 2$ parameters. More specifically, the model preserves the symmetry of the direct interaction of two opinions postulated in the adaptive voter model, i.e. in direct comparison no opinion is stronger than the other. However, it breaks the symmetry between different pairings of opinions such that rewiring is more likely in certain pairings than in others.

In Section 3.1.2 we saw that the fragmentation transition separates an active regime, where a finite density of active links persists (until fluctuations drive the system eventually to an absorbing consensus state), from a fragmented regime, where disconnected components emerge. Because the $G$-state model contains several different types of active links (corresponding to all possible pairings in $\Gamma$ ), regimes can occur where active links of a certain type vanish while others prevail. This can lead to configurations where a certain subset of the states only appears in one component of the network in which no state not belonging to this subset is present. In the following we call this situation a partially fragmented state.

In contrast to the fully fragmented state, where every component is internally in consensus, the dynamics in the partially fragmented state can continue in some components while others may be frozen in internal consensus. The partial fragmentation cannot be undone, so that achieving global consensus is impos- 


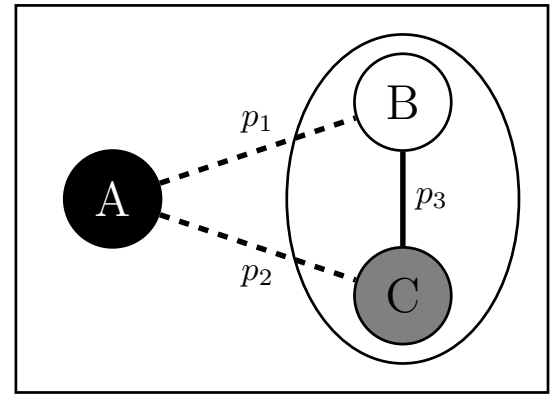

Figure 3.8: State-network of a 3-state voter model. The nodes in this network correspond to three opinions $A, B$ and $C$. The rewiring rates $p_{1}, p_{2}$ or $p_{3}$, encode the degree of controversy between agents holding the respective pairings of opinions $A B, B C$ or $C A$. The ellipse and dashed lines illustrate the example described in the text: partial fragmentation with respect to $A$.

sible after partial fragmentation has occurred. However, the ongoing dynamics in the active components will eventually lead to an absorbing state in every component. The absorbing state which is ultimately reached after a partial fragmentation therefore in general consists of $1<\gamma<G$ major components, holding the $\gamma$ surviving opinions, respectively. For $\gamma=G$ we recover full fragmentation and the case where $\gamma=1$ we denote as the fully active regime where all types of active links prevail. Only in the latter case (due to finite-size effects) global consensus can be reached eventually.

\subsubsection{Fragmentation transitions in a 3-state model}

We start our exploration of the proposed multi-state voter model by considering the case $G=3$, as this is the simplest case which is not trivial $(G=1)$ or extensively studied $(G=2)[73,114-116]$. Let us consider the set of opinions $\Gamma=$ $\{A, B, C\}$, giving rise to three different rewiring rates $\left\{p_{A B}, p_{A C}, p_{B C}\right\}$ which we denote as $\left\{p_{1}, p_{2}, p_{3}\right\}$ according to the state-network depicted in Fig. 3.8.

In principle, in the limit of large $N$ a 3 -state system can reach five different final states: a fully active regime, full fragmentation and partial fragmentation with respect to $A, B$, or $C$. Here, partial fragmentation with respect to a certain state refers to a situation where a component of nodes in that particular state fragments from an active component (a mixed component of nodes in the other states).

For the calculation of the fragmentation threshold, i.e. the transition from a fully active to a fragmented regime, we follow the approach described in Section 3.2. We determine the evolution equations for the number of active motifs starting from a situation close to the fragmentation threshold. For simplicity, 
we only use the fan motif set here. We emphasize that all calculations below can also be carried out using more elaborate motif bases, but at the price of having to deal with considerably larger matrices.

Previously, we defined $q$-fans as a bundle of $q$ active links emanating from the focal node. Recall that we do not account for the number of inert links connecting to this focal node. Because in a 3-state model we have three types of active links, the set of $q$-fans in principle consists of all possibilities for choosing $q$ active links from three types of active links. For simplicity, we consider only pure fans, i.e. fans which contain only one type of active link. For example, an $A B$-fan is a bundle of $A B$-links, connected to a single $A$ - or $B$-node. We confirmed that the incorporation of mixed fans barely influences the results.

This can be understood as follows: In the case where $p_{3}$ is close to or beyond the fragmentation threshold, it is obvious that the probability to create a mixed fan goes to zero. If $p_{3}$ is much below the fragmentation threshold, mixed fans tend to be converted into pure fans before the next update occurs on an $A B$-or $A C$-fan. This is because we assume a situation where only few active links of type $A B$ and $A C$ are left, and therefore almost all updates occur on $B C$-links. Most of those updates are opinion adoption events and thus tend to destroy mixed fans. For $p_{3} \approx 0$ and for large mean degrees, the deviation might become significant. In this case, one can incorporate mixed fans in order to obtain more accurate results.

We start by calculating the condition for partial fragmentation with respect to $A$ (see Fig. 3.8). The $A$-cluster fragments from the rest of the network when all $A B$-motifs and all $A C$-motifs vanish. Because in general $p_{1} \neq p_{2}$, we have to treat $A B$ - and $A C$-motifs separately. We start by considering a network with two almost disconnected clusters, one of which is composed purely of $A$-nodes and the other one of $B$ - and $C$-nodes. Then, we ask whether the fragmented state is stable, such that fragmentation is reached, or unstable, such that the system avoids fragmentation.

In the almost fragmented state the expected effect of network updates on the active motifs is captured by the procedure described above. For the case of $k=3$ we obtain the transitions rules shown in Fig. 3.9. New active motifs are created when an opinion update occurs. We approximate the degree of the focal node $k$ by the networks mean degree $\langle k\rangle$. Because of the clusters being almost-separated the newly formed active motif is a $k-1$-fan. This fan can subsequently lose active links due to subsequent opinion updates and rewiring events. We account for a finite density of active $B C$-links in the active component (captured by $\rho_{3}$ ) by creating an $A C$-fan ( $A B$-fan) instead of an $A B$-fan $\left(A C\right.$-fan) with probability $\rho_{3}$ when a new fan is created by a $B$-node ( $C$-node) adopting opinion $A$. Starting with equal distribution of states, the relation $\rho_{3}=[B C] /(k[B])=[B C] /(k[C])$ holds, where $[B]$ and $[C]$ denote the numbers of $B$-nodes and $C$-nodes, respectively. Note that $\rho_{3}$ differs from the global $B C$-link density $\rho_{3}^{(G)}=[B C] / L$. 


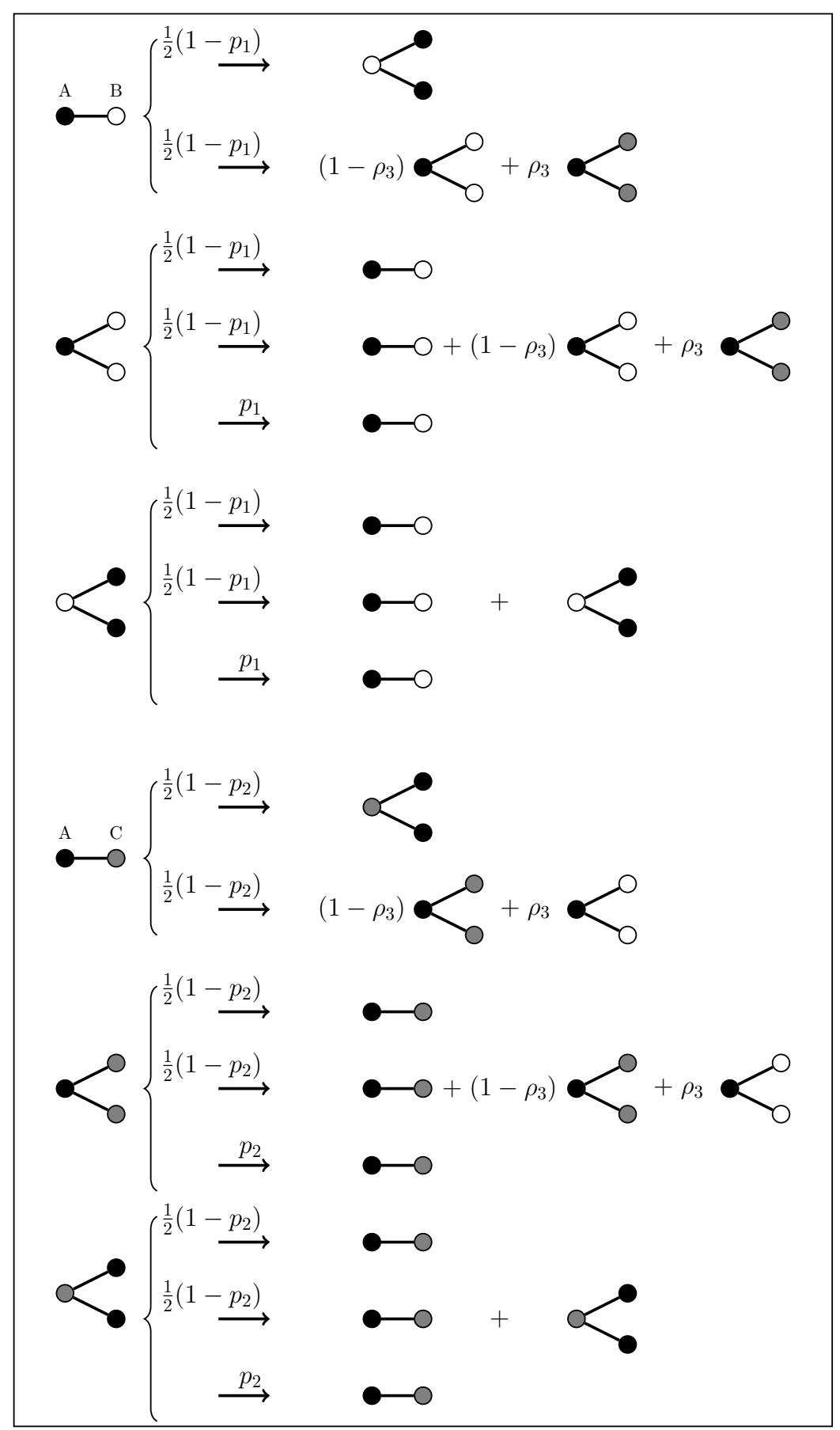

Figure 3.9: Transitions of $A B$ - and $A C$-fans for a degree-regular network with $k=3$ for the scenario of partial fragmentation with respect to A. Black, white and grey nodes correspond to agents holding opinion $A, B$, and $C$, respectively. The active link densities $\rho_{1}$ and $\rho_{2}$ are assumed to vanish close to the partial fragmentation point, whereas $\rho_{3}$, the density of $B C$-links is possibly finite. 


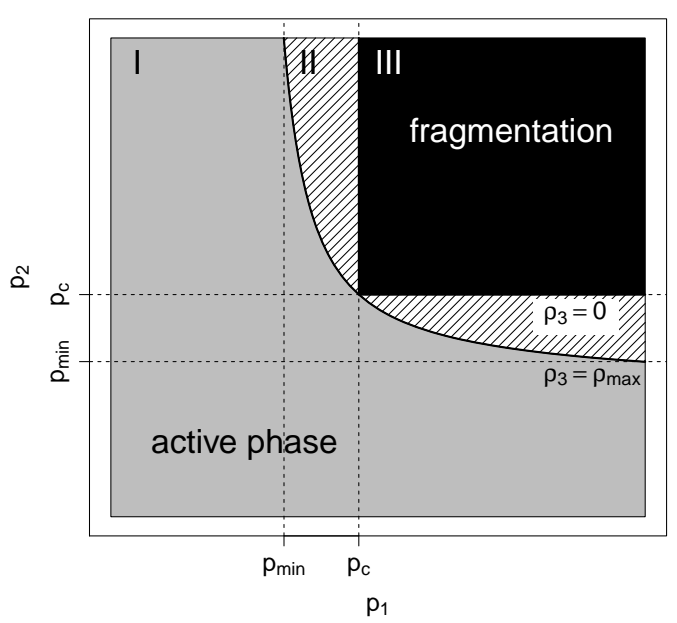

Figure 3.10: Schematic fragmentation diagram for a 3-state system. The region where fragmentation with respect to $A$ occurs depends on the $B C$-link density $\rho_{3}$ : If $\rho_{3}=0$, fragmentation with respect to $A$ occurs only in region III. If $\rho_{3}>0$, fragmentation with respect to $A$ can occur additionally in part of the dashed region. For $\rho_{3}=\rho_{\max }$ the maximal extension of the fragmentation region (II + III) is realized. In region $\mathrm{I}$, no fragmentation with respect to $A$ occurs. If $p_{1}>p_{3}$ and $p_{2}>p_{3}$, this fragmentation diagram suffices to determine the final state of the whole system.

The set of transitions for $k=3$ (see Fig. 3.9) defines a dynamical system, describing the time evolution of the densities of active motifs close to partial fragmentation with respect to $A$. The stability of the partially fragmented state in this system is governed by the block-structured Jacobian matrix,

$$
\mathbf{J}\left(p_{1}, p_{2}, \rho_{3}\right)=\left(\begin{array}{cc}
\mathbf{D}_{p_{1}}-\mathbf{X}_{p_{1}}\left(\rho_{3}\right) & \mathbf{X}_{p_{1}}\left(\rho_{3}\right) \\
\mathbf{X}_{p_{2}}\left(\rho_{3}\right) & \mathbf{D}_{p_{2}}-\mathbf{X}_{p_{2}}\left(\rho_{3}\right)
\end{array}\right)
$$

where

$$
\mathbf{D}_{p_{i}}=\left(\begin{array}{ccc}
-1 & \frac{1}{2}\left(1-p_{i}\right) & \frac{1}{2}\left(1-p_{i}\right) \\
1 & \frac{1}{2}\left(1-p_{i}\right)-1 & 0 \\
1 & 0 & \frac{1}{2}\left(1-p_{i}\right)-1
\end{array}\right)
$$

and

$$
\mathbf{X}_{p_{i}}\left(\rho_{j}\right)=\left(\begin{array}{ccc}
0 & \frac{1}{2}\left(1-p_{i}\right) \rho_{j} & 0 \\
0 & \frac{1}{2}\left(1-p_{i}\right) \rho_{j} & 0 \\
0 & 0 & 0
\end{array}\right)
$$

The diagonal blocks in the Jacobian matrix given in Eq. (3.4) can be interpreted as "self-interaction" terms, capturing contributions from the same motif-type, 
and the off-diagonal terms as "exchange" terms, capturing contributions from different motif-types. The structure of this Jacobian matrix remains unchanged for any partial fragmentation of a 3-state system, while the matrices (3.5) and (3.6) change when the motif set is altered. In particular, the dimension of these matrices increases with increasing mean degree and/or number of motifs considered.

The partially fragmented state is stable if all eigenvalues of the Jacobian matrix $\mathbf{J}$ are negative. Therefore, demanding $\lambda(\mathbf{J})=0$, where $\lambda(\mathbf{J})$ is the leading eigenvalue of $\mathbf{J}$, yields a condition for the fragmentation transition, depending on the three parameters $p_{1}, p_{2}$, and $\rho_{3}$. The phase diagram in Fig. 3.10 is a projection of this fragmentation condition on the $p_{1}-p_{2}$-plane for the extreme values of $\rho_{3}$.

Let us first consider the case where $\rho_{3}=0$, which is encountered if $p_{3}$ exceeds $p_{c}$, the fragmentation threshold of the adaptive 2-state voter model. In this case, $\mathbf{X}$ becomes zero and the set of eigenvalues of the Jacobian matrix $\mathbf{J}$ is the conjunction of the eigenvalues of the matrices $\mathbf{D}_{p_{1}}$ and $\mathbf{D}_{p_{2}}$. Thus, $\lambda(\mathbf{J})$ is negative if $\lambda\left(\mathbf{D}_{p_{1}}\right)$ and $\lambda\left(\mathbf{D}_{p_{2}}\right)$ are negative. Indeed matrices $\mathbf{D}_{p_{1}}$ and $\mathbf{D}_{p_{2}}$ are the Jacobian matrices of the two uncoupled 2-state systems $A-B$ and $A-C$. Thus fragmentation of $A$ requires that the 2 -state fragmentation condition is met separately for the $A B$ - and $A C$-subsystems. In other words, if the links between $B$ and $C$ nodes vanish $\left(\rho_{3}=0\right)$, fragmentation occurs when both $p_{1}>p_{c}$ and $p_{2}>p_{c}$ (see Fig. 3.10 region III).

For studying the case $\rho_{3}>0$ we first note that every matrix-valued row of J sums to $\mathbf{D}_{p_{i}}$, where $i=1,2$. Below, when we consider the general case of $G$ states, we show that these rowsums imply bounds for $\lambda(\mathbf{J})$. More precisely, $\lambda(\mathbf{J})$ is bounded by $\lambda\left(\mathbf{D}_{p_{1}}\right)$ and $\lambda\left(\mathbf{D}_{p_{2}}\right)$. Therefore, fragmentation with respect to $A$ is guaranteed when $p_{1}>p_{c}$ and $p_{2}>p_{c}$ (region III), but can already occur when only either $p_{1}>p_{c}$ or $p_{2}>p_{c}$ is satisfied (region II).

The maximal extension of region II is observed when $p_{3}=0$. The corresponding maximal value $\rho_{3}=\rho_{\max }$ can be determined to good approximation by a first order moment closure approach (pair-approximation). To illustrate this, let us calculate the maximal active link density in an active cluster of $s$ states in a $G$-state system. If we assume $p_{i j}=0$ for all rewiring rates within the active cluster, the evolution equation for the number of active links of type $x y$ is given by

$$
[\dot{x y}]=-[x y]+\frac{1}{2}\left(\sum_{z \neq x}[x z y]+\sum_{z \neq y}[x z y]-\sum_{z \neq y}[x y z]-\sum_{z \neq x}[z x y]\right) .
$$

Then, using the pair-approximation formulae given in (2.9), the number of active 
links in the steady-state yields,

$$
[x y]=\frac{1}{2}\left(\sum_{z \neq x, y} 2 \frac{[x z][z y]}{[z]}+2 \frac{[x y][y y]}{[y]}+2 \frac{[x x][x y]}{[x]}-\sum_{z \neq y} \frac{[x y][y z]}{[y]}-\sum_{z \neq x} \frac{[z x][x y]}{[x]}\right) .
$$

For equal distribution of states we can write for any $x$ and $y$ :

$$
[x]=\frac{n}{s}, \quad[x y]=\zeta, \quad[x x]=\eta,
$$

where $n$ denotes the number of nodes in the active component. The total number of links $l$ in the active component is in this notation given by

$$
l=s \eta+\frac{s(s-1)}{2} \zeta
$$

which yields for (3.7)

$$
\zeta=\frac{\zeta^{2}}{n} s(s-2)+2 \frac{\zeta l}{n}-2 \frac{\zeta^{2}}{n} s(s-1)
$$

With $k n=2 l$, we find for the maximum active link density in a cluster of $s$ states,

$$
\rho_{\max }=\frac{s(s-1) \zeta}{2 l}=\frac{(s-1)(k-1)}{s k} .
$$

For three states, we have $s=2$, so that the maximal active link density yields in this case

$$
\rho_{\max }=\frac{k-1}{2 k} .
$$

Note that (3.8) is in agreement with the expression for two states given in (3.1) for $p=0$ and $\langle k\rangle=k$, because the active subsystem consists of two states, $B$ and $C$.

Now, solving the condition $\lambda\left(\mathbf{J}\left(p_{1}, p_{2}, \rho_{\max }\right)\right)=0$ numerically yields the curve separating regions I and II in Fig. 3.10. Moreover, from the diagram in Fig. 3.10 it is clear that this curve implies the existence of a minimal rewiring rate $p_{\min }$, such that for $p_{1}<p_{\min }$ or $p_{2}<p_{\text {min }}$ partial fragmentation with respect to $A$ becomes impossible, in the infinite size limit.

Let us emphasize, that calculations of fragmentation thresholds for partial fragmentations build on the estimation of the active link densities from given rewiring rates. As there is no accurate analytical expression for $\rho(p)$ in the whole $p$-range (see [115] and Section 3.4.1), the long-term behavior can only be predicted with certainty in regions I and III of the fragmentation diagram.

Evaluating the partial fragmentation condition with respect to $A$, i.e. $\lambda(\mathbf{J})=0$ with $\mathbf{J}$ given in (3.4), leads to a phase diagram like the one shown in Fig. 3.10, 

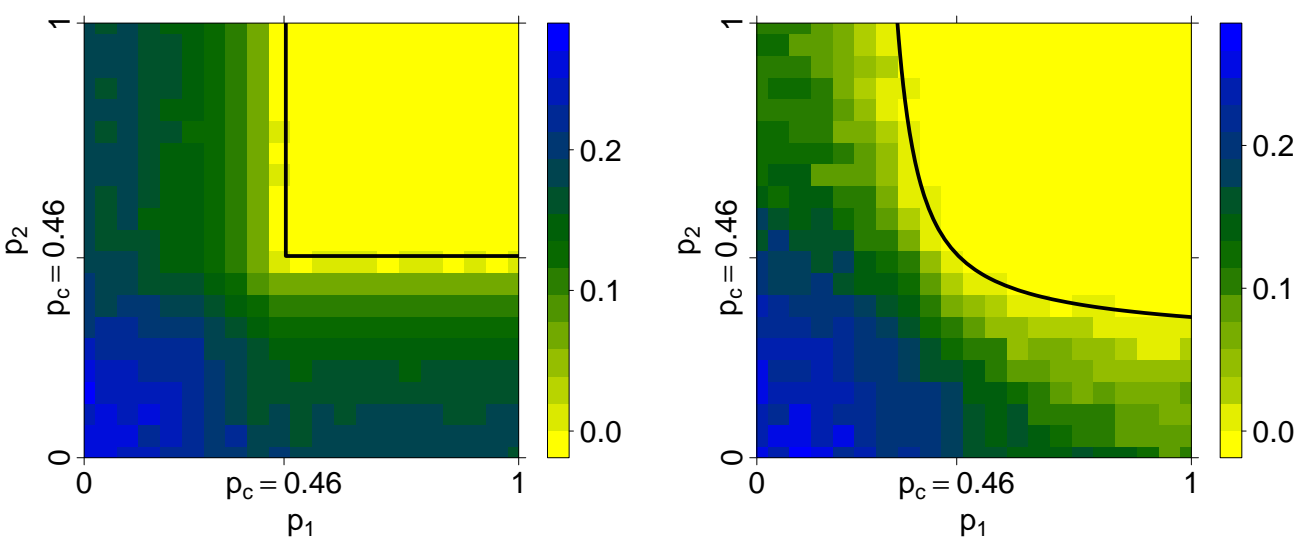

Figure 3.11: Numerical phase diagram for the 3-state model. Plotted is the density of links, connecting the $A$ and the $B C$-cluster $\left(\rho_{1}+\rho_{2}\right)$ over the rewiring rates $p_{1}$ and $p_{2}$. Light regions correspond to fragmentation with respect to $A$. The left panel shows the case $\rho_{3}=0\left(p_{3}=0.5\right)$. This corresponds to an uncoupled system: the critical rewiring rates for $p_{1}$ and $p_{2}$ are the same as for the 2-state voter model with the same mean degree, $p_{c}=0.46$. In the right panel, $\rho_{3}$ is maximal $\left(p_{3}=0\right)$. Here, the active link density in the active cluster leads to an extension of the fragmentation region. Black lines represent analytical results. Link densities were measured at $t=1000$, i.e. long before the active phase decays due to finite-size effects. $N=10000,\langle k\rangle=4$, averaged over 20 realizations.

where three different regions can be distinguished: In regions I and III partial fragmentation occurs or is avoided regardless of $p_{3}$, whereas in region II partial fragmentation depends on $\rho_{3}$ and consequently on the setting of the related rewiring rate $p_{3}$. We found that these results are in very good agreement with data obtained from agent-based simulations of large networks (Fig. 3.11). In order to extract the transition between fragmentation and active phase from simulations of a finite network, one has to consider a timeframe where the system is far from reaching a finite-size induced absorbing state (see [118] for a more detailed discussion of the finite-size behavior).

So far, we studied partial fragmentation with respect to one specific state (state $A$ ). In order to predict the final state of the whole system, one has to analyze the corresponding partial fragmentation diagrams for each of the three states. Let us assume $p_{1} \geq p_{2} \geq p_{3}$. Then, there are four cases to distinguish (see Fig. 3.12): 


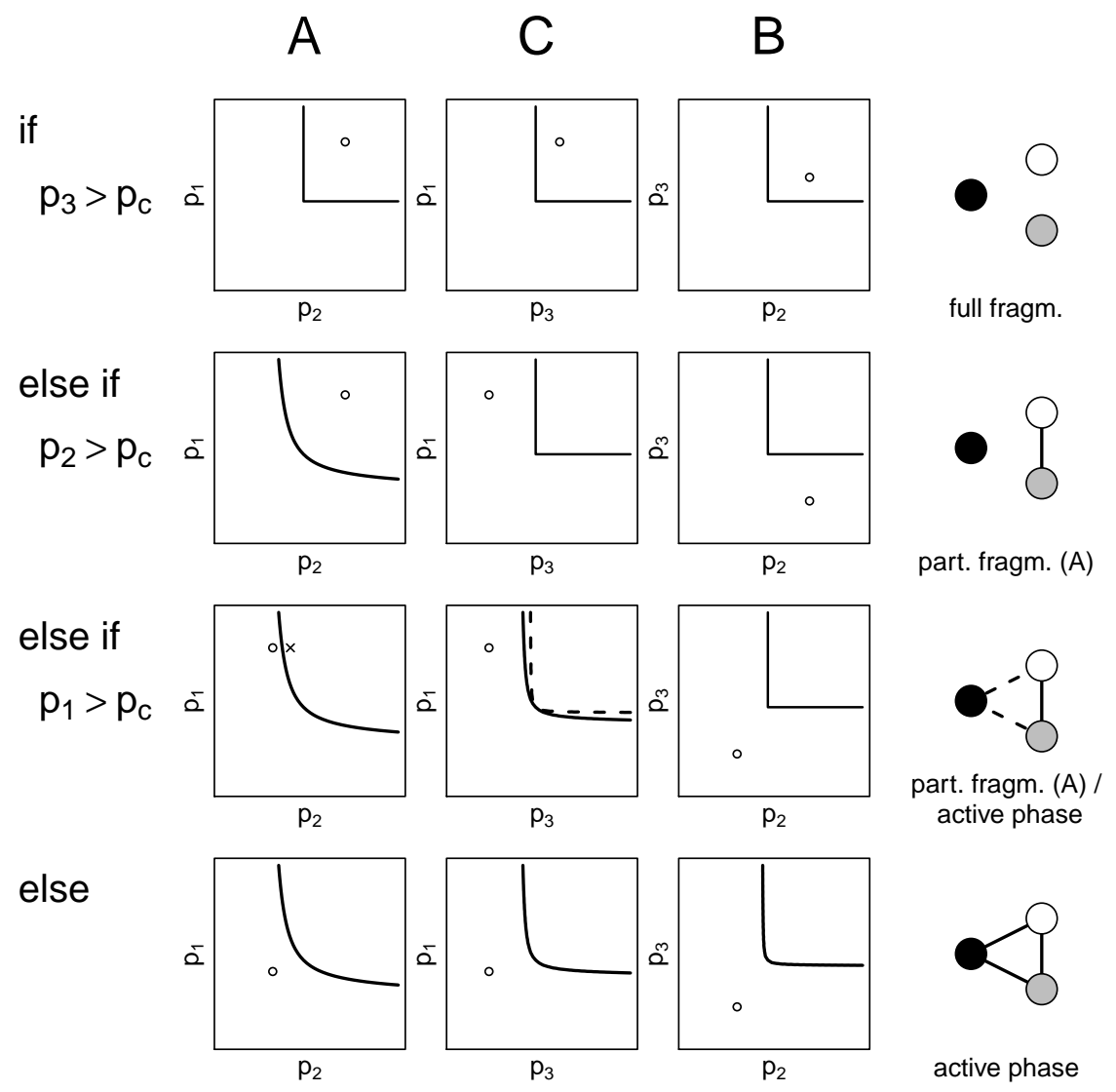

Figure 3.12: Fragmentation diagrams for partial fragmentation with respect to state $A, C$ or $B$ for $p_{1} \geq p_{2} \geq p_{3}$ in the limit of infinite network size. There are four different cases, according to the conditions given on the left-hand side of the chart. The positions of the points $P=\left(p_{2}, p_{1}\right), Q=\left(p_{3}, p_{1}\right), R=\left(p_{2}, p_{3}\right)$ indicate for each case whether partial fragmentation is reached for the respective state. The final state of the whole system can be deduced from the outcomes for all three states and is given on the right-hand side of the chart. For the first, second and forth case the final state can be predicted without ambiguity. In the third case, either partial fragmentation with respect to $A$ or an active equilibrium can be reached, depending on the specific parameter setting (the two possibilities are indicated by two different symbols $\circ$ and $\times$ in the first diagram and a solid and a dashed line in the second diagram of the third row). It can be seen that partial fragmentation is only possible with respect to the state with the largest rewiring rates (here $A$ ). 
1. $p_{3}>p_{c}$,

2. $p_{3}<p_{c}$ and $p_{2}>p_{c}$,

3. $p_{2}<p_{c}$ and $p_{1}>p_{c}$

4. $p_{1}<p_{c}$.

In case 1) full fragmentation is reached, because all points $P=\left(p_{2}, p_{1}\right), Q=$ $\left(p_{3}, p_{1}\right)$ and $R=\left(p_{2}, p_{3}\right)$ lie in the region III of their respective diagrams. In case 2) $P$ lies in III and $Q$ and $R$ in I. Thus, partial fragmentation with respect to $A$ occurs, while $B$ - and $C$-nodes form an active cluster and in a finite system eventually reach consensus. In case 3 ) the point $P$ lies either in region I or II of the corresponding fragmentation diagram for $A$, whereas $Q$ is always in region I, because $p_{2} \geq p_{3}$ and $\rho_{3} \geq \rho_{2}$. This means that in this case either partial fragmentation with respect to $A$ occurs or the system reaches an active phase, depending on the specific values of $p_{1}, p_{2}, p_{3}$. In case 4) $P, Q$ and $R$ lie in the region I of their respective diagrams, implying that the active phase is reached. Note that this shows that partial fragmentation can only occur with respect to that state, which is connected via the largest rewiring rates to the two other states in the state network.

In summary, we showed that in the 3-state voter model either consensus, partial fragmentation or full fragmentation occurs. Full fragmentation is only reached when all rewiring rates exceed $p_{c}$. Analyzing the phase diagram with respect to the state which is connected via the largest rewiring rates to the other states suffices for the prediction of the final state of the whole system. For quantitative predictions in region II of the diagram the active link density corresponding to the lowest rewiring rate has to be known. Qualitatively, one can say that whenever partial fragmentation occurs, it is with respect to the "most different" state.

\subsubsection{Fragmentation transitions in a $G$-state model}

When we consider a general system of $G$ states, in contrast to the 3-state model, partial fragmentations can also occur with respect to a group of states. A general multi-state network can thus fragment into several active components. In the following, we calculate the condition for a system to fragment into two components containing $s$ and $G-s$ states, respectively (see Fig. 3.13). This is in principle no restriction, as a fragmentation into more than two components can be treated as a fragmentation into two components where the active components in their turn fragment.

For clarity we only use one level of indices from now on: we write $\mathbf{D}_{i j}$ and $\mathbf{X}_{i j}$ instead of $\mathbf{D}_{p_{i j}}$ and $\mathbf{X}_{p_{i j}}$. Furthermore, in order to distinguish indices which 
refer to one component from those referring to the other component we use indices $i \in\{1, \ldots, s\}$ for the component with $s$ states and indices $\underline{i} \in\{\underline{1}, \ldots, \underline{s}\}$ for the component with $\underline{s}=G-s$ states. For example, the inter-component rewiring rates are then denoted as $p_{i \underline{i}}$ and intra-component active link densities as $\rho_{i j}$ and $\rho_{i j}$, respectively.

In analogy to the treatment of the 3-state model, we consider a situation where the two clusters are almost fragmented. We then determine the evolution equations for a set of active motifs connecting the two components. In the 3state case these were of two types, $A B$ - and $A C$-fans, which led to a Jacobian matrix of $2 \times 2$ matrix-valued entries and a fragmentation condition which was a function of the rewiring rates $p_{1}$ and $p_{2}$ and the active link density $\rho_{3}$. In the general case the Jacobian matrix contains $s \underline{s} \times s \underline{s}$ matrix-valued entries, according to the $s \cdot \underline{s}$ inter-component links in the state-network (see Fig. 3.13) and the fragmentation condition is a function of all inter-component rewiring rates $\left\{p_{i \underline{i}}\right\}$ and all intra-component active link densities $\left\{\rho_{i j}\right\}$ and $\left\{\rho_{i j}\right\}$.

Following the same procedure as for the 3 -state system, one finds that the general Jacobian matrix exhibits a block-structure of $s \times s$ submatrices,

$$
\mathbf{J}\left(p_{i \underline{i}}, \rho_{i j}, \rho_{\underline{i j}}\right)=\left(\begin{array}{cccc}
\boldsymbol{\Delta}_{1}\left(\rho_{i \underline{j}}, \rho_{1 j}\right) & \xi_{1}\left(\rho_{12}\right) & \cdots & \xi_{1}\left(\rho_{1 s}\right) \\
\xi_{2}\left(\rho_{21}\right) & \boldsymbol{\Delta}_{2}\left(\rho_{i \underline{j}}, \rho_{2 j}\right) & \ddots & \vdots \\
\vdots & \ddots & \ddots & \xi_{(s-1)}\left(\rho_{(s-1) s}\right) \\
\xi_{s}\left(\rho_{s 1}\right) & \ldots & \xi_{s}\left(\rho_{s(s-1)}\right) & \boldsymbol{\Delta}_{s}\left(\rho_{\underline{i j}}, \rho_{s j}\right)
\end{array}\right),
$$

where $\boldsymbol{\Delta}_{i}$ and $\xi_{i}$ are matrices of $\underline{s} \times \underline{s}$ matrix-valued entries,

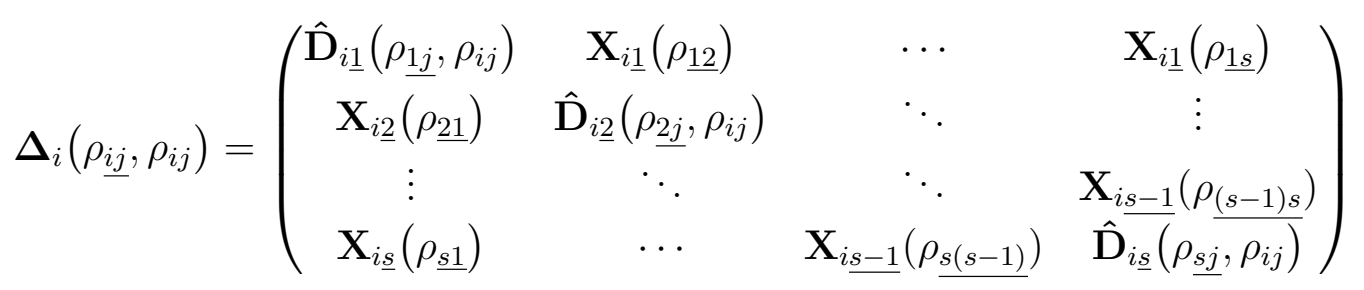

and

$$
\xi_{i}\left(\rho_{i j}\right)=\left(\begin{array}{cccc}
\mathbf{X}_{i \underline{1}}^{\prime}\left(\rho_{i j}\right) & \mathbf{0} & \cdots & \mathbf{0} \\
\mathbf{0} & \mathbf{X}_{i \underline{2}}^{\prime}\left(\rho_{i j}\right) & \ddots & \vdots \\
\vdots & \ddots & \ddots & \mathbf{0} \\
\mathbf{0} & \cdots & \mathbf{0} & \mathbf{X}_{i \underline{s}}^{\prime}\left(\rho_{i j}\right)
\end{array}\right)
$$

Here, we introduced the abbreviation

$$
\hat{\mathbf{D}}_{i \underline{i}}\left(\rho_{\underline{i j}}, \rho_{i j}\right)=\mathbf{D}_{i \underline{i}}-\sum_{\underline{j}=\underline{1}, \underline{j} \neq \underline{i}}^{\underline{s}} \mathbf{X}_{i \underline{i}}\left(\rho_{\underline{i j}}\right)-\sum_{j=1, j \neq i}^{s} \mathbf{X}_{i \underline{i}}^{\prime}\left(\rho_{i j}\right) \text {. }
$$


For $k=3$, the matrices $\mathbf{D}$ and $\mathbf{X}$ were given in (3.5) and (3.6) and the matrix $\mathbf{X}^{\prime}$ yields

$$
\mathbf{X}_{i \underline{i}}^{\prime}\left(\rho_{i j}\right)=\left(\begin{array}{ccc}
0 & 0 & \frac{1}{2}\left(1-p_{i \underline{i}}\right) \rho_{i j} \\
0 & 0 & 0 \\
0 & 0 & \frac{1}{2}\left(1-p_{i \underline{i}}\right) \rho_{i j}
\end{array}\right) .
$$

The latter matrix appears for fragmentations where both of the fragmenting components are active, i.e. for $1<s<G-1$.

Note that every matrix-valued row of the Jacobian matrix sums to $\mathbf{D}_{i i}$ and refers to one specific type of inter-component link with rewiring rate $p_{i i}$ in the state-network. One such row thus represents the transitions for one motif-type. Entries $\hat{\mathbf{D}}$ on the diagonal capture the creation of motifs of the same type, while off-diagonal entries $\mathbf{X}$ and $\mathbf{X}^{\prime}$ denote transitions to different motif-types, which arise from the intra-component link densities $\rho_{i j}$ and $\rho_{i j}$, respectively. For example, the entries in one row describing the transitions of $g_{1} g_{\underline{2}}$-fans depend on the rewiring rate between the states $g_{1}$ and $g_{2}$, which is $p_{1 \underline{2}}$, the active link densities between $g_{1}$ and all other states in the first component, $\rho_{1 x}$, with $x \in\{2, \ldots, s\}$; and the active link densities between $g_{2}$ and all remaining states in the second component, $\rho_{2 y}$, with $y \in\{\underline{1}, \underline{3}, \ldots, \underline{s}\}$.

In analogy to the 3 -state model the active link densities $\rho_{i j}$ entering in the Jacobian matrix relate to the global active link density $\rho_{i j}^{(G)}$ as

$$
\rho_{i j}=\frac{\left[g_{i} g_{j}\right]}{k\left[g_{i}\right]}=\frac{G}{2} \frac{\left[g_{i} g_{j}\right]}{L}=\frac{G}{2} \rho_{i j}^{(G)}
$$

and analogously for $\rho_{i j}$.

Stability analysis of the general Jacobian matrix in (3.9) leads to a fragmentation condition which depends directly or indirectly (through the active link densities) on all $G(G-1) / 2$ different rewiring rates. In contrast to the estimation of the active link density in a 2-state system, in a multi-state system the active link density of a certain link-type does not only depend on the rewiring rate of that specific link-type, but also on the rewiring rates and active link densities of the neighboring links in the state-network. Inferring the link densities analytically from the rewiring rates is presently an unsolved challenge (see also Section 3.4.2). So, even for given rewiring rates it is in general not possible to make quantitative predictions about fragmentation thresholds. Nevertheless, the structure of the Jacobian matrix reveals some further insights about fragmentation transitions, as we will see in the following.

Therefore, let us recall some theorems about upper and lower bounds of the largest eigenvalue $\lambda(\mathbf{M})$ of a nonnegative irreducible matrix $\mathbf{M}$. The well-known Frobenius inequality states (see e.g. [133])

$$
\min _{k} S_{k} \leq \lambda(\mathbf{M}) \leq \max _{k} S_{k}
$$




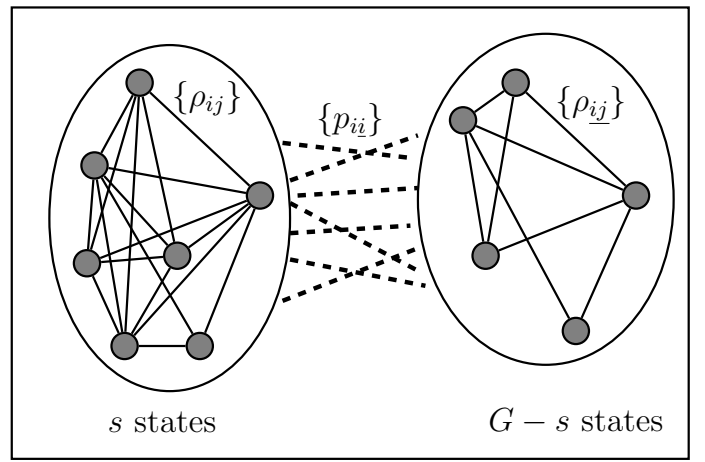

Figure 3.13: Schematic representation of a state-network with $G$ states. Ellipses illustrate a fragmentation into two (possibly) active clusters of $s$ and $G-s$ states. Dashed lines correspond to inter-cluster links with rewiring rates $\left\{p_{i \underline{i}}\right\}$, connecting every state in one cluster with every state in the other cluster. The number of inter-cluster links, $s \cdot(G-s)=s \cdot \underline{s}$, determines the dimension of the (matrix-valued) Jacobian matrix. Solid lines correspond to intra-cluster links within both clusters with active link densities $\left\{\rho_{i j}\right\}$ and $\left\{\rho_{\underline{i j}}\right\}$, respectively.

where $S_{i}$ is the rowsum of the $i$-th row of $\mathbf{M}$. A generalization of the above inequality for a partitioned nonnegative irreducible square matrix $\mathbf{M}$ is given in [134]. Let us assume that $\mathbf{M}$ can be partitioned into square submatrices $\mathbf{M}$ ij, such that

$$
\mathbf{M}=\left(\begin{array}{cccc}
\mathbf{M}_{11} & \mathbf{M}_{12} & \cdots & \mathbf{M}_{1 N} \\
\mathbf{M}_{21} & \mathbf{M}_{22} & \cdots & \mathbf{M}_{2 N} \\
\vdots & \vdots & \ddots & \vdots \\
\mathbf{M}_{N 1} & \mathbf{M}_{N 2} & \cdots & \mathbf{M}_{N N}
\end{array}\right)
$$

We define generalized, matrix-valued, rowsums

$$
\mathbf{S}_{k}=\sum_{j=1}^{N} \mathbf{M}_{k j}, \quad k=1, \ldots N .
$$

Then, the following inequality holds [134]:

$$
\lambda\left(\min _{k} \mathbf{S}_{k}\right) \leq \lambda(\mathbf{M}) \leq \lambda\left(\max _{k} \mathbf{S}_{k}\right)
$$

The expressions $\min _{k}$ and $\max _{k}$ have to be understood element-wise, i.e. the matrix $\min _{k} \mathbf{S}_{k}$ is the matrix which is obtained when we take element-wise the minimum over all $\mathbf{S}_{k}$ and analogously for the maximum.

In the following, we apply the theorem quoted above to the Jacobian matrix given in (3.9). This is possible because $\mathbf{J}$ can be written as $\mathbf{J}=\mathbf{T}-\mathbf{1}$, where $\mathbf{T}$ 
is a nonnegative irreducible matrix and $\mathbf{1}$ is the identity matrix of appropriate dimension. We will consider two different partitions.

First, let us consider a partition (P1) of the Jacobian matrix into $s \underline{s}$ submatrices. Then, the matrix-valued rowsums corresponding to this partition yield

$$
\begin{aligned}
\mathbf{S}_{k}^{(\mathrm{P} 1)} & =\hat{\mathbf{D}}_{i \underline{i}}\left(\rho_{\underline{i j}}, \rho_{i j}\right)+\sum_{\underline{j}=\underline{1}, \underline{j} \neq \underline{\underline{i}}}^{\underline{s}} \mathbf{X}_{i \underline{i}}\left(\rho_{\underline{i j}}\right)+\sum_{j=1, j \neq i}^{s} \mathbf{X}_{i \underline{i}}^{\prime}\left(\rho_{i j}\right) \\
& =\mathbf{D}_{i \underline{i}}, \quad k=1, \ldots, s \underline{s} .
\end{aligned}
$$

The matrices $\mathbf{D}_{i \underline{i}}$ only depend on $p_{i \underline{i}}$ and it can be seen from (3.5) that all non-constant entries in $\mathbf{D}_{p}$ increase with decreasing $p$. Therefore, we get for the upper and lower bounds of $\lambda(\mathbf{J})$

$$
\lambda\left(\mathbf{D}_{p_{\max }}\right) \leq \lambda(\mathbf{J}) \leq \lambda\left(\mathbf{D}_{p_{\min }}\right),
$$

where

$$
p_{\max }=\max _{i, \underline{i}} p_{i \underline{i}}, \quad p_{\min }=\min _{i, \underline{i}} p_{i \underline{i}} .
$$

From (3.13) we deduce the following statements:

- When all inter-cluster rewiring rates $p_{i i}$ are below the threshold $p_{c}$, no fragmentation occurs, because $\lambda\left(\mathbf{D}_{p_{\max }}\right)>0$.

- When all inter-cluster rewiring rates $p_{i \underline{i}}$ exceed the threshold $p_{c}$, fragmentation occurs, because $\lambda\left(\mathbf{D}_{p_{\min }}\right)<0$.

- If $p_{\min }=p_{\max }$, necessarily all inter-cluster rewiring rates must be equal. In that case, the fragmentation condition is the classical condition of the 2-state voter model, $\lambda(\mathbf{J})=\lambda\left(\mathbf{D}_{p}\right)=0$, which yields the critical rewiring rate $p_{c}$.

The first two results represent an intuitive generalization of our findings for the 3 -state case. The last result implies that if all inter-cluster rewiring rates are equal then the value of these rewiring rates, $p$, is the only parameter, which the fragmentation transition depends on. In this case a precise analytical estimation of the fragmentation point is possible because the active link densities arising from the intra-cluster links, do not enter. Furthermore, note that this result is independent of the number of opinions. This implies that in the special case of equal inter-cluster rewiring rates, systems of any size behave identically to a properly-initialized adaptive 2 -state voter model.

We test the latter result in a 5 -state system, considering a fragmentation into two components of two and three states, respectively (inset in Fig. 3.14). Simulations show that for randomly chosen rewiring rates $r_{i}$ within the two clusters, 


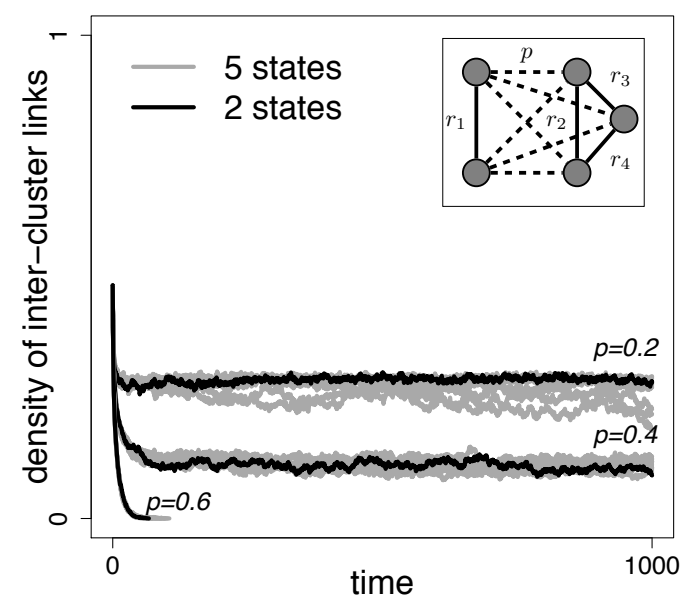

Figure 3.14: Numerical test of the reduction principle. We run simulations for a 5 -state system (inset) assigning random values to the rewiring rates $r_{i}$. Plotted is the density of inter-cluster links (dashed links) for three different values of $p$ (and ten independent simulations each). For comparison, the corresponding active link density of a 2 -state model with the same rewiring rate and a ratio $2: 3$ for the number of nodes in opposite states is shown. It can be seen that the inter-cluster density does not depend on $r_{i}$ and that it reaches a steadystate value corresponding to the inter-cluster density (active link density) in the adaptive 2 -state voter model. $N=10000,\langle k\rangle=4$.

the inter-cluster link density reaches the same steady-state value (Fig. 3.14). A further comparison shows, that the behavior of a 5-state model closely matches the behavior of a 2-state model.

Now we consider another partition, (P2), of the Jacobian matrix, which is a partition into $s$ submatrices. Then, the corresponding generalized rowsums yield

$$
\begin{aligned}
\mathbf{S}_{i}^{(\mathrm{P} 2)} & =\Delta_{i}\left(\rho_{\underline{i j}}, \rho_{1 j}\right)+\sum_{j=1, j \neq i}^{s} \xi_{i}\left(\rho_{i j}\right) \\
& =\left(\begin{array}{cccc}
\tilde{\mathbf{D}}_{i \underline{1}}\left(\rho_{\underline{1 j}}\right) & \mathbf{X}_{i \underline{1}}\left(\rho_{\underline{12}}\right) & \ldots & \mathbf{X}_{i \underline{1}}\left(\rho_{\underline{1 s}}\right) \\
\mathbf{X}_{i \underline{2}}\left(\rho_{\underline{11}}\right) & \tilde{\mathbf{D}}_{i \underline{2}}\left(\rho_{2 \underline{j}}\right) & \ddots & \vdots \\
\vdots & \ddots & \ddots & \mathbf{X}_{i \underline{s-1}}\left(\rho_{(s-1) s}\right) \\
\mathbf{X}_{i \underline{s}}\left(\rho_{\underline{s 1}}\right) & \cdots & \mathbf{X}_{\underline{i s-1}}\left(\underline{\left.\rho_{\underline{s(s-1)}}\right)}\right. & \overline{\mathbf{D}}_{i \underline{s}}\left(\rho_{\underline{s j}}\right)
\end{array}\right),
\end{aligned}
$$


where

$$
\tilde{\mathbf{D}}_{i \underline{i}}\left(\rho_{\underline{i j}}\right)=\mathbf{D}_{i \underline{i}}-\sum_{\underline{j}=\underline{1}, \underline{j} \underline{i} \underline{\underline{s}}}^{\underline{s}} \mathbf{X}_{i \underline{i}}\left(\rho_{\underline{i j}}\right) .
$$

First, we observe that every matrix $\mathbf{S}_{i}^{(\mathrm{P} 2)}$ corresponds to a partial fragmentation in a system of $\bar{s}+1$ states. More precisely, the set $\left\{\mathbf{S}_{i}^{(\mathrm{P} 2)}\right\}$ describes a collection of $s$ single-state-fragmentations where for every $i$ a single state $g_{i}$ is taken separately from the $s$-cluster. This single state (i.e. now $s=1$ ) then forms the first component of the partial fragmentation, while the second component is given by the whole $\underline{s}$-cluster.

Now, building the element-wise extrema of $\left\{\mathbf{S}_{i}^{(\mathrm{P} 2)}\right\}$ means to compare all the single-state-fragmentations by comparing every matrix-entry of the corresponding generalized rowsums. Taking $\min _{i} \mathbf{S}_{i}^{(\mathrm{P} 2)}\left(\max _{i} \mathbf{S}_{i}^{(\mathrm{P} 2)}\right)$ yields therefore in every matrix-entry the minimum (maximum) value, i.e. that one which comprises the maximal (minimal) rewiring rate. The resulting matrix corresponds to a partial fragmentation with respect to a single state where the inter-cluster rewiring rates are chosen extremal according to the described comparison. We will refer to such a system as bounding system (see Fig. 3.15 for exemplary bounding systems).

For a partition of type (P2) the leading eigenvalue of the general Jacobian matrix satisfies

$$
\lambda\left(\min _{i} \mathbf{S}_{i}^{(\mathrm{P} 2)}\right) \leq \lambda(\mathbf{J}) \leq \lambda\left(\max _{i} \mathbf{S}_{i}^{(\mathrm{P} 2)}\right) .
$$

The lower bound corresponds to the fragmentation of a system where the largest inter-cluster rewiring rate of each state in the second component is connected to a single state. The upper bound corresponds to the fragmentation of a system where the smallest inter-cluster rewiring rate of each state in the second component is connected to a single state.

As in the Jacobian matrix in (3.9) the matrices $\mathbf{X}$ and $\mathbf{X}^{\prime}$ can be interchanged, one can consider a corresponding partition where the second component is reduced to a single state, i.e. $\underline{s}=1$ and the first component remains unchanged. This leads to a different set of bounding systems, as illustrated in Fig. 3.15 a) and $b)$.

From (3.14) we can draw the following conclusions:

- If the lower bounding system does not fragment $\left(\lambda\left(\min _{i} \mathbf{S}_{i}^{(\mathrm{P} 2)}\right)>0\right)$, the original system does not fragment.

- If the upper bounding system fragments $\left(\lambda\left(\max _{i} \mathbf{S}_{i}^{(\mathrm{P} 2)}\right)<0\right)$, the original system fragments.

- If upper and lower bounding systems coincide $\left(\min _{i} \mathbf{S}_{i}^{(\mathrm{P} 2)}=\max _{i} \mathbf{S}_{i}^{(\mathrm{P} 2)}\right)$, the fragmentation of the original system is exactly described by the Jacobian matrix of the bounding system. 


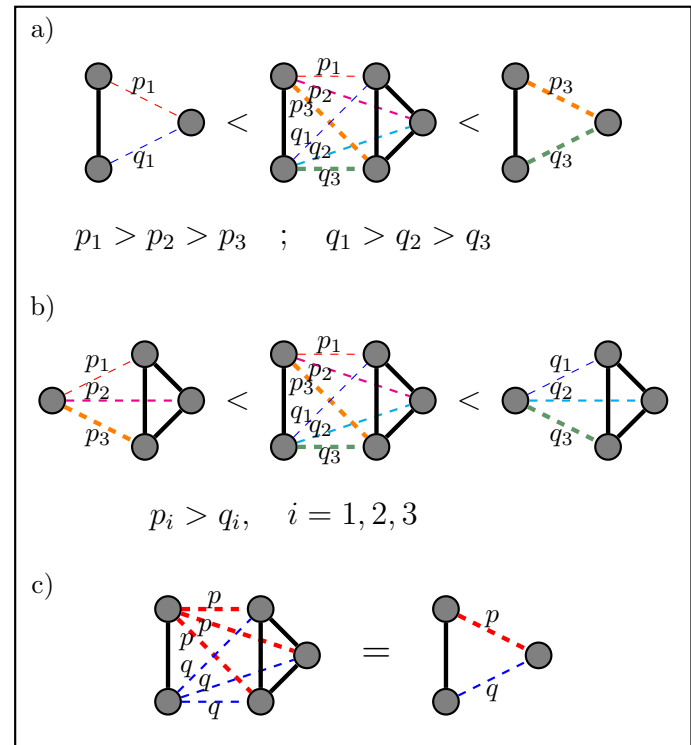

Figure 3.15: Schematic representation of the generalized Frobenius inequalities for a 5-state system and a partial fragmentation into a 2-state- and a 3 -state-cluster. Comparisons refer to the corresponding largest eigenvalues of the Jacobian matrices describing the indicated fragmentations. The inequalities in a) and b) represent two different sets of bounding systems obtained from a partition of type (P2), as described in the text. In c) a special case of a) is shown, where the upper and lower bounding systems coincide. In this case the considered fragmentation of the original system is fully captured by the corresponding fragmentation in the lower-dimensional system.

The latter case is realized if every state in one component is connected via equal rewiring rates to every state in the other component (see Fig. 3.15 c)). For statenetwork topologies which display this property the dimension of the Jacobian matrix reduces significantly and thus the fragmentation condition becomes much more tractable.

The results from the second partition show that for the leading eigenvalue of a Jacobian matrix, corresponding to a partial fragmentation into two active clusters, upper and lower bounds can be given, which correspond to singlestate-fragmentations in (properly constructed) lower-dimensional systems. In particular, the leading eigenvalue of the full Jacobian matrix can be exactly calculated as the leading eigenvalue of a lower-dimensional Jacobian matrix if special state-network topologies are given. Otherwise, when such a reduction is not possible, the bounding systems provide necessary conditions for a partial fragmentation to occur. So, calculating fragmentation conditions for the much 
simpler bounding systems in some cases suffices to predict the occurrence or absence of fragmentations in the full system.

To summarize, in this section we extended the analysis of fragmentation transitions to a family of multi-state voter models. For the 3-state model our analysis revealed a phase diagram in which three distinct types of behavior are observed. Depending on the parameters the system either approaches a consensus state, a partially fragmented state ultimately leading to two surviving opinions or a fully fragmented state in which all three opinions survive.

In a general scenario with an arbitrary number of states making precise predictions is more difficult. In particular, the computation of fragmentations generally requires the estimation of active link densities inside the clusters between which the fragmentation occurs. By exploiting the specific structure of transition rates in the system, one can nevertheless gain analytical insights into the fragmentation dynamics. For example we identified a class of special cases in which adaptive multi-state voter models exactly recover the behavior of the adaptive 2-state voter model.

While the ultimate goal of understanding opinion formation in the human population is still far away, the presented model illustrates how analytical understanding can be pushed to more complex models.

\subsection{Further applications of the percolation approach}

In this last section of Chapter 3, we briefly discuss two further applications of the proposed percolation approach. First, we illustrate an application to directed networks by calculating the fragmentation point in a directed adaptive voter model. Then, we demonstrate how the approach can be used to estimate the active link density in the original adaptive voter model.

\subsubsection{Directed voter model}

In the following, we consider fragmentation transitions in a directed voter model. From the methodological point of view, this model is beneficial for testing the validity of the percolation approach twofold: 1) Showing the applicability of the approach to directed network models means a significant broadening of the class of models which can be treated with the percolation approach. 2) The directed voter model can be used for testing the performance of our approach for broad degree distributions.

From the sociological point of view, the directed voter model incorporates one additional feature, which is likely to be present in real societies, namely the directionality of influence. A directed link in the directed voter model thus encodes who is influenced by whom or, in the context of Twitter, "who follows whom " [117]. The additional feature of directionality makes it therefore easier to 
compare analytical and numerical results obtained from the model to data from online social communities, a subject which has recently gained much attention $[92,96,135]$.

In the directed voter model, we start with $N$ nodes, $L$ directed links and an equal distribution of node states, say $A$ and $B$. Every node $i$ holds a state $s_{i} \in\{A, B\}$. Then, in one update step a random link $i \rightarrow j$ is chosen. With probability $p$ the link is removed and a new link between $i$ and a randomly chosen node $k$ with $s_{k}=s_{i}$ is established. With probability $1-p$, node $i$ adopts the state of node $j$, such that $s_{i}$ is set to $s_{j}$.

The rewiring rule assures a constant out-degree distribution, which means that the number of outgoing links of each node remains unchanged, while the in-degree distribution becomes Poissonian due to the random rewiring process. This allows for studying the impact of different out-degree distributions on the (fragmentation) dynamics of the model. In the undirected voter model, an arbitrary initial degree distribution becomes roughly Poissonian due to the rewiring process, impeding the analysis of more realistic degree distributions.

Note that here, in contrast to the update rule for the original adaptive voter model introduced in Section 3.1, an update also affects inert links. This slight difference in the update rule prevents a so-called early fragmentation [117], which cannot be described by the percolation approach. Here, we stick to the discussion of implications of directionality and degree distribution for the prediction of fragmentation transitions. For an extensive analysis of the directed voter model, in particular the effect of early fragmentation, we refer to [117].

For the calculation of the fragmentation point in the directed voter model, the procedure is very similar to the one outlined in Section 3.2: Assuming a network configuration close to the fragmentation point, we evaluate the transition equations for active motifs regarding their stability.

Here, we choose spider motifs as a basis. Because the center node of a spider motif can only switch its opinion when an opinion update on one of its outgoing active links occurs, and a new spider motif can only arise if an opinion update on one of its incoming active links occurs, spider motifs have to track four types of links: outgoing and incoming inert links and outgoing and incoming active links. Thus, a specific spider motif is characterized by a 4-tuple capturing the numbers of the four types of links. In Fig. 3.16 the transitions for a general spider motif close to the fragmentation point are illustrated. The prefactors in the transition probabilities account for the probability of choosing an active incoming link out of all active links and the probability of choosing an active outgoing link out of all active links, respectively.

We find that for Poissonian in- and out-degree distributions the predicted fragmentation point matches quite closely the critical point obtained from simulations. For example, for $\langle k\rangle=8$ the percolation approach yields $p_{c} \cong 0.8$, while in simulations $p_{c} \cong 0.78$ is observed [117]. So, the directionality of the 


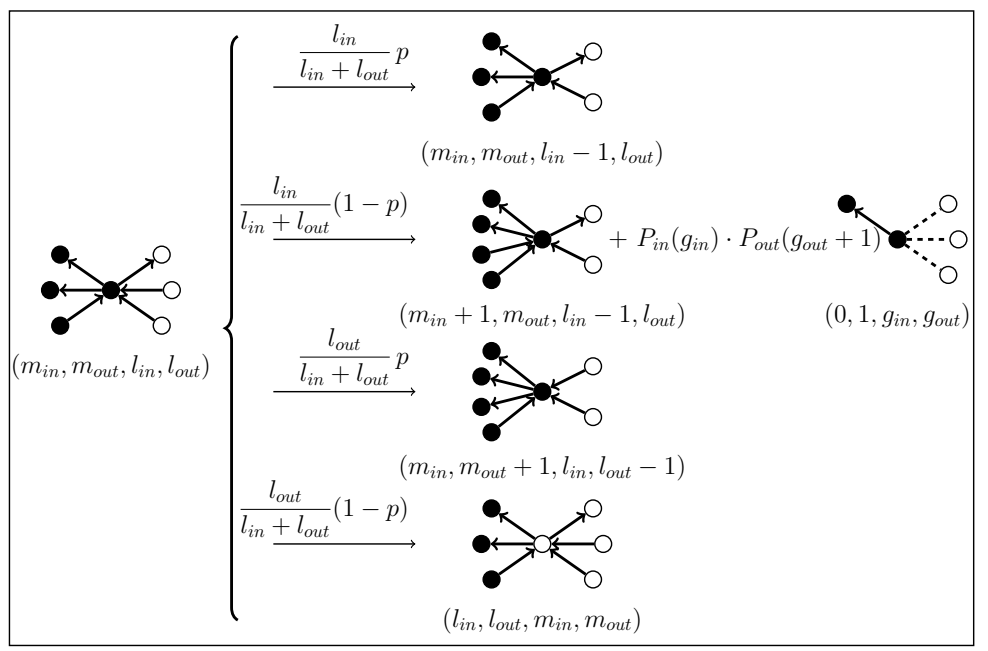

Figure 3.16: Schematic of the transitions of spider motifs in a directed voter model. In order to account for incoming and outgoing active links and incoming and outgoing inert links, every motif is characterized by a 4-tuple $\left(m_{\text {in }}, m_{\text {out }}, l_{\text {in }}, l_{\text {out }}\right)$. The probability for the creation of a new spider of a certain type is determined by the in- and out-degree distributions $P_{\text {in }}$ and $P_{\text {out }}$. Here, we assume that the in- and out-degree of a node is uncorrelated, so that the joint probability becomes the product of the respective individual probabilities $P_{\text {in }} \cdot P_{\text {out }}$.

network does not implicate any difficulties in applying the percolation approach, despite the fact that the dimension of the transition matrix becomes larger as we have to distinguish between incoming and outgoing links in the spider motifs.

It turns out that the effect of the degree distribution on the calculation of the fragmentation point is more significant [117]. In particular, considering broad degree distributions comes along with a loss of precision of the fragmentation point. However, this is not a conceptual problem of the approach, but rather due to limited computational power. For broad degree distributions the cut-off, i.e. the maximal out-degree which is taken into account, would ideally chosen to be equal to the largest out-degree in the actual network. But, for the directed voter model the dimension of the Jacobian increases quadratically with the cutoff (see Fig. 3.17). This behavior becomes clear when doing the combinatorics for the number of different 4-tuples corresponding to given $k_{\text {out }}^{\max }$ and $k_{\text {in }}^{\max }$. We conclude that there is a trade-off between precision and computational effort, which becomes particularly relevant for broad degree distributions and large networks. 


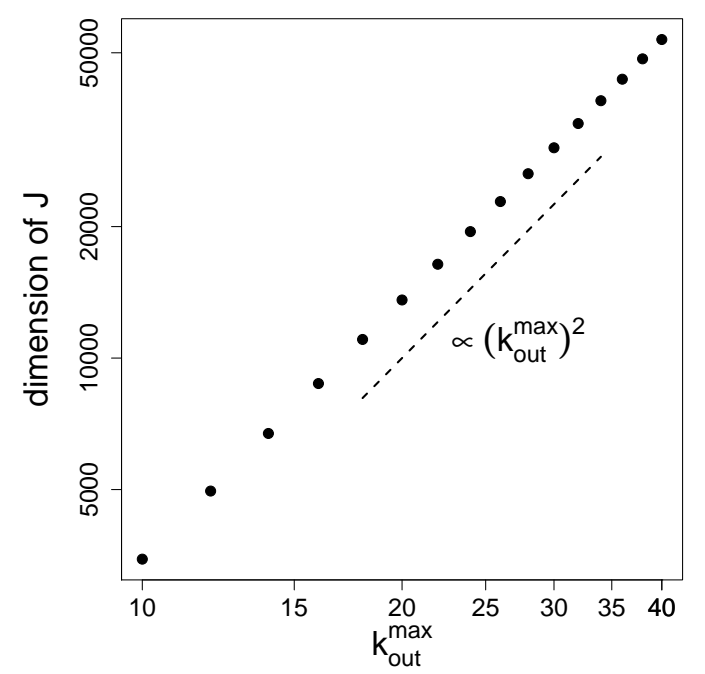

Figure 3.17: Dimension of the Jacobian in dependence of the cut-off degree. The dimension increases for increasing $k_{\text {out }}^{\max }$ and fixed $k_{\text {in }}^{\max }=10$ approximately quadratically. For comparison, the dashed line shows $\left(k_{\text {out }}^{\max }\right)^{2}$. Diagonalisation of the corresponding large matrices constitutes the major limitation for the precision of the fragmentation point in networks with scale-free out-degree distributions.

The example discussed in this section shows in which respect the percolation approach can be used for more involved models and where limitations arise. We saw that directionality can be immediately implemented in the approach, while the drawback of considering broad degree distributions lies in the difficulty to choose an adequate cut-off degree.

\subsubsection{Estimation of active link density}

As a last application of the percolation approach, let us again consider the original two-state voter model introduced in Section 3.1. In (3.1) an expression for the active link density $\rho$ as a function of the rewiring rate $p$ was given, which can be obtained from a first order moment closure approach (pair-approximation). For equal distribution of states $(\alpha=0.5)$ relation (3.1) reads

$$
\rho=\frac{\langle k\rangle(1-p)-1}{2\langle k\rangle(1-p)} .
$$

The corresponding curve for $\langle k\rangle=4$ is plotted in Fig. 3.19 (dashed line). It can be seen that this theoretical estimation for the active link density matches the simulation results (black dots) quite well for $p \ll p_{c}$, while for rewiring rates close 


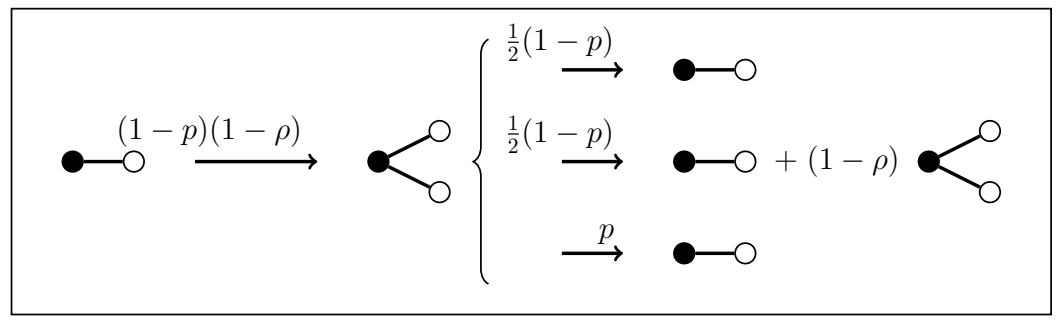

Figure 3.18: Transitions for fan motifs in a degree-regular network with $k=$ $\langle k\rangle=3$. In contrast to Fig. 3.4, here we assume that neighboring motifs are already activated with probability $\rho$, so that in an opinion-adoption event they might become deactivated. As a consequence, new 2-fans are created with decreased probability. This variation allows for an estimation of the active link density (away from the fragmentation point).

to the fragmentation point it fails drastically. As mentioned earlier, the reason for this failure is due to the assumption made in mean-field-like approaches, that active links are homogeneously distributed in the system throughout the whole $p$-range. Indeed, homogeneity in the active link distribution is only warranted for relatively low rewiring rates, but is destroyed for $p$-values close to the fragmentation point [115].

In the following, we demonstrate how the percolation approach introduced in Section 3.2 can be used to obtain an alternative estimate of the active link density. Therefore, we use a variant of the procedure described in Section 3.2. Now, we account for the presence of active links within the two components, which were previously assumed to be homogeneous. This implies that the activation of new active motifs becomes less probable as there is a non-zero probability of deactivating an already active motif, instead.

For illustration, let us consider again the degree-regular case where $k=\langle k\rangle=$ 3, and assume a set of fan motifs (Fig. 3.18). A single active link gives rise to a 2 -fan with probability $1-p$, provided that the neighboring links are not active already. If we assume $\rho$ to be the probability for both links to be active, then the probability of creating a 2 -fan in an update-event of a single active link becomes $(1-p)(1-\rho)$. Similarly, in the second transition, the creation of a new 2 -fan out of a 2 -fan occurs with probability $(1-p)(1-\rho) / 2$. Here, we made the assumption that neighboring fan motifs are either completely active or completely inactive, inspired by the observation that active links tend to cluster close to the fragmentation point [115].

The transitions in Fig. 3.18 lead to the following Jacobian matrix,

$$
\mathbf{J}=\left(\begin{array}{cc}
-1 & (1-p)(1-\rho) \\
1 & -1+\frac{1}{2}(1-p)(1-\rho)
\end{array}\right)
$$




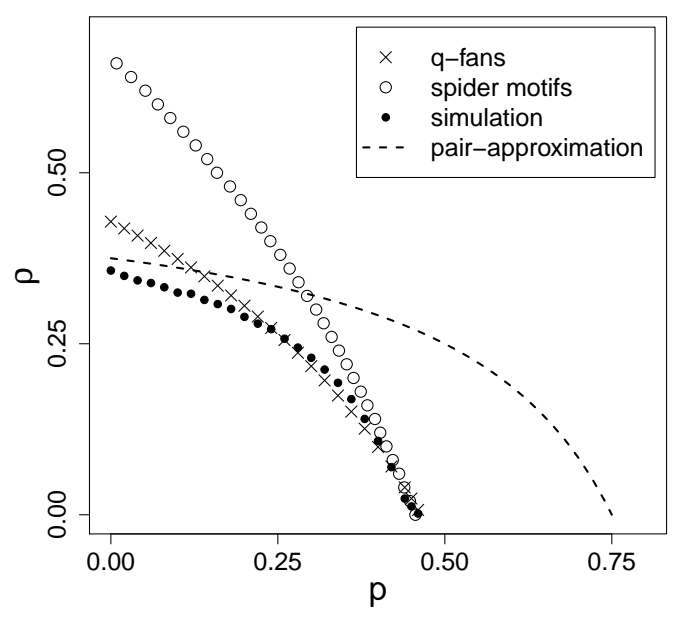

Figure 3.19: Active link density in the adaptive voter model. For comparison, we show the results obtained from simulations $(\bullet)$, from the percolation approach with $q$-fans $(\times)$, from the percolation approach with spider motifs (o) and from pair-approximation (dashed line). Note that spider motifs, which yield more accurate results for the fragmentation point, estimate the active link density for $p<p_{c}$ worse than $q$-fans. $N=10^{5},\langle k\rangle=4$.

from which we obtain a condition for the active steady state $\lambda(\mathbf{J}(p, \rho))=0$ and therefore an expression for the active link density in the steady state,

$$
\rho(p)=\frac{1-3 p}{3(1-p)} .
$$

The corresponding curve for $\langle k\rangle=4$ is shown in Fig. $3.19($ symbol $\times)$. We find that the active link density obtained from a percolation approach yields, in contrast to the estimation from pair-approximation, a very good agreement with simulation results close to the fragmentation point, while it becomes less accurate for low values of $p$. The latter observation is expected, as we used features (e.g. clustering of active links) which are specifically present in the vicinity of the fragmentation point. Analogously, it turns out that for increased precision of the fragmentation point, i.e. by taking the spider motif set, the estimation of the active link becomes worse for $p<p_{c}$, compared to the fan motif set (see Fig. 3.19).

Note the similarity of the matrix in (3.16) to previously discussed cases: 1) For $\rho=0$ the matrix becomes the Jacobian matrix in (3.3), which determines the stability of the fragmented state. Consequently, one can interprete the matrix in (3.16) as the Jacobian matrix governing the stability of the active 
steady state. 2) The matrix in (3.16) structurally corresponds to the diagonal "self-interaction" terms in the Jacobian matrix of the 3-state model, (3.4). The only difference is that in (3.4), $\rho$ refers to the link density of $B C$-links, while in (3.16), the link density of $A B$-links itself is denoted by $\rho$. Consequently, in the latter case, whenever an already active motif is met, it vanishes (becomes inert) with probability $(1-p) / 2$ while in the multi-state model it becomes an active motif of different type. So, formally we can represent the matrix for the active link density in (3.16) as a Jacobian matrix describing a partial fragmentation into two $A B$-clusters.

To summarize, a variant of the percolation approach which accounts for additional active motifs within the fragmenting components, allows for an estimation of the active link density in the adaptive voter model. For rewiring rates close to $p_{c}$ the obtained estimate from this procedure is quite accurate, while for low rewiring rates pair-approximation yields more precise results (see Fig. 3.19). How to properly combine both approaches in order to obtain a reasonable expression for the active link density in the whole $p$-range is presently an unsolved challenge. A heuristic way to do so, is to construct a function $\rho(p)$ which smoothly extrapolates between $\rho_{\mathrm{MC}}(p)$ for small $p$ and $\rho_{\mathrm{PA}}(p)$ for large $p .^{7}$ However, even though the resulting curve matches the simulation results better than any of the original curves, it is rather artificial and does not provide insight about the actual process going on in the system. The goal is to describe the transition from a regime where active links are homogeneously distributed $(p \approx 0)$ to a regime where active links are highly clustered $\left(p \approx p_{c}\right)$. Ideally, one would find a mathematical description for the aggregation process of active links in dependence on the rewiring rate $p$.

\subsection{Discussion}

In this chapter, we developed a method for the analytical estimation of fragmentation thresholds and tested the performance of our approach in specific examples. Fragmentation transitions are a common feature of adaptive networks for opinion formation and characterize a transition from a global homogeneous state to a fragmented state where diverse opinions coexist in separate components. From a sociological point of view, it is desirable to understand phenomena such as the formation of subpopulations or the establishment of global consensus. A mathematical description of the phenomenon is crucial for the investigation of existing and upcoming models. As the widely used moment closure approximation yields poor estimates for fragmentation thresholds, there had been no analytical approach available which faithfully predicted the fragmentation point.

\footnotetext{
${ }^{7}$ Here, by $\rho_{\mathrm{MC}}(p)$ we denote the moment closure result from $(3.15)$ and by $\rho_{\mathrm{PA}}(p)$ we denote the percolation approach result, which is for $\langle k\rangle=3$ given in (3.17) and which can be analogously obtained for arbitrary $\langle k\rangle$.
} 
Inspired by the idea of percolation, we proposed an alternative approach where we derived evolution equations for a set of active motifs assuming a network configuration close to the fragmentation point. Then, evaluating the stability of the fragmented state yields an estimate for the fragmentation threshold. We find that this estimate is much more precise than predictions from moment closure approximations. We believe that the main reason for the good performance of the percolation approach is that, in contrast to mean-field-like approximations, our approach takes clustering of active links close to the fragmentation point into account.

The fact that active links are concentrated at few network nodes is captured in the choice of the basis set. We presented two possible bases for the derivation of our system of equations. The first one, the basis of $q$-fans allows for a quick and reasonable estimation of the fragmentation point, whereas the second one, the spider motif set, comprises more computational effort but allows for a very precise prediction of the fragmentation point.

After introducing the percolation approach in detail using the example of an adaptive voter model, we demonstrated its applicability to more elaborate models for opinion formation.

First, we considered a multi-state voter model, where agents choose an opinion from a discrete set of arbitrary-many opinions. Using the percolation approach, we were able to analyze partial fragmentations of the network, and we identified a class of equivalent models with respect to fragmentations. If there exists an equivalent model of lower dimension than the original system, the analysis of fragmentation transitions simplifies considerably.

Then, we studied fragmentations in an adaptive voter model on a directed network. This model is particularly instrumental for testing the performance of our approach with respect to broad degree distributions. We pointed out that the cut-off degree influences the precision of the estimated fragmentation point to large extent, which makes it computationally costly to obtain good results for scale-free networks.

Finally, we used the percolation approach to calculate the active link density in the original adaptive voter model. As expected, the obtained expression is reasonable for rewiring rates close to the fragmentation threshold. For rewiring rates much smaller than $p_{c}$ the predicted active link density overestimates the actual value. Now, a challenge for future work is to combine the mean-field picture, which is valid for small rewiring rates, with the picture of highly clustered and localized active links, which is true close to the fragmentation point, in order to obtain an analytical description of the active link density in the whole parameter range. Our approach, and the reasoning which led to its development comprise an important contribution to this currently unsolved problem.

To summarize, our approach provides a framework for the calculation of fragmentation thresholds, which is quite general. The crucial prerequisite is that 
the update rule is based on a contact process. Otherwise, if active links arise spontaneously, the fragmented state is not absorbing and clustering of active links cannot be assured. The percolation approach in principle allows for the treatment of many variants of the adaptive voter model, including direct and reverse node update [29] or different rewiring schemes [111, 112]. Furthermore, it can be used for the analysis of fragmentations in more realistic models, which account for asymmetric relationships between opinions (biased voter model), intrinsic preferences [136], bounded confidence [105, 106, 137], the influence of many neighbors in the same state [138], and similar refinements. In specific cases, further information about degree distributions and (degree-) correlations have to be considered. 


\section{Persistence of complex food webs in metacommunities}

Ecological systems are complex systems which typically offer a high degree of diversity. A central aim of ecology is to understand the emergence of the enormous diversity of ecological species in order to facilitate its maintenance. Therefore, it is conducive to study the conditions for emergence and loss of diversity in model systems. In this chapter, we use a network approach to investigate conditions for the coexistence of different species in ecological communities.

In a network description of an ecological system, the network nodes represent single organisms, whole species, or entire populations composed of several species, depending on the level of coarse graining. Among these options, a commonly chosen representation of ecological systems is a description in terms of food webs [139-144].

Food webs are networks where nodes correspond to species and directed links correspond to trophic interactions, pointing from a resource species to a predator. Such predator-prey interaction patterns can be obtained from observations and analyzed with respect to their size, structure and connectivity. In particular, it has been found that most food webs observed in nature comprise a large number of different species and exhibit a high level of complexity [145-148].

Recent investigations have identified several factors that contribute to the stability of large food webs and thus promote diversity [149-153]. However, growing evidence suggests that, at least in some systems, the food web emerges only on a regional scale, whereas simple food chains are observed if specific locations (patches) are considered in isolation. This points to a need for a nonlocal perspective, in which one explicitly accounts for the spatial distribution of resource patches and dispersal of species across these patches.

Then, the question arises, how different communities can be sustained in a network of similar patches. This question was first discussed in the context of metapopulation theory, introduced in [154, 155]. Metapopulation models consider several species which spread over a system of identical patches, such that each patch is either occupied by one species or vacant. It was found that, due to spatial dispersal, different species can coexist at the metapopulation level, even though individual patches only host one species type.

A similar feature was observed for a class of metacommunity models that was recently proposed in [156-158]. In metacommunity theory, the metapopulation framework is extended to account for predator-prey interactions. Thus, various trophicaly interacting species can inhabit a single patch. Due to dispersal across patches, and as different compositions of species may be realized in different patches, a complex interaction pattern can arise at the metacommunity level. 
In other words, metacommunity theory describes an ecological system as a network of networks, where the food webs encoding trophic interactions within patches are coupled via a patch-network, representing the spatial dispersal routes of species. It is self-evident that the emergent food web at the metacommunity scale, which in general differs from the local food webs within the patches, depends both, on the structure of the predator-prey interactions, and on the structure of the patch-network. In this chapter we develop a mathematical framework which facilitates the analysis of these two types of dependencies.

We start in Section 4.1 with a detailed description of the metacommunity model. Then, using as an example a simple linear food web, we illustrate the idea of our mathematical approach for the calculation of persistence conditions. In Section 4.2 we extend our approach and calculate persistence conditions for predators in more complex food webs. The obtained conditions reveal explicit relations between species diversity and food web topology. In Section 4.3 we study the influence of the patch-network on species diversity. We consider patch-networks with multiple types of habitat and patch-networks which exhibit heterogeneous degree distributions. We summarize and discuss our results in Section 4.4.

\subsection{Introduction to metacommunity theory}

In the present chapter, we employ a metacommunity model which has recently been proposed by Pillai et al. [156-158]. This model is relatively simple and therefore conducive to a detailed analysis. A crucial assumption of the model is that within each patch competitive exclusion precludes the formation of complex food webs. This means, within patches the model does not allow for more than one predator feeding on the same prey species. Thus any single patch can only harbor a food chain. However, as different food chains may be realized in different patches a complex food web emerges at the system level (see Fig. 4.1).

In this section, after introducing the model in detail, we focus on the emergence of linear chains in the metacommunity. Such food chains are the simplest form of emergent food webs. We use this simple example as an introduction and for illustration of our method, which we subsequently apply to more complex food webs in Section 4.2.

\subsubsection{Definition of the model}

Following $[156,157]$, we consider a metacommunity consisting of $s$ species which populate a network of interconnected patches. The proposed model does not account for the abundance of a species $i \in\{1,2, \ldots, s\}$ found in a specific patch, but captures only the presence (or absence) of this species by a boolean variable. Thus, every patch can either be empty or occupied by a set of species. 


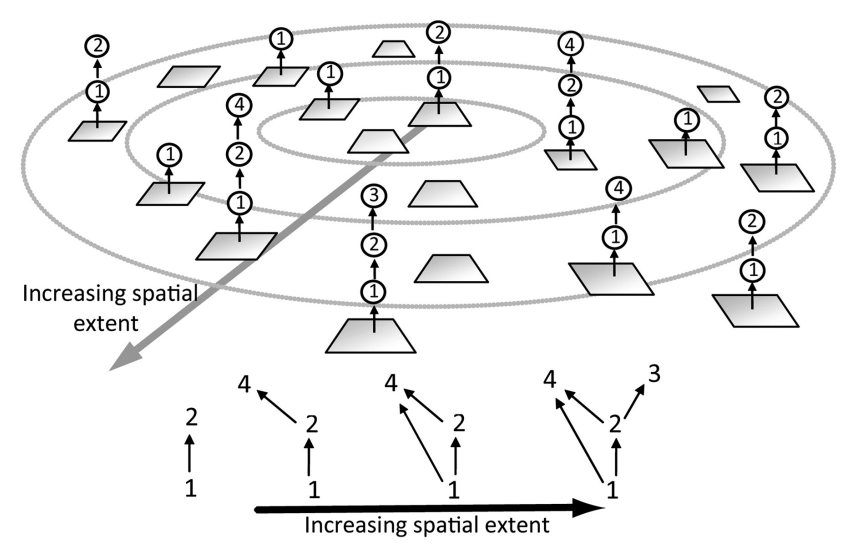

Figure 4.1: Schematic illustration of the emergence of complex food webs. Spatially coupled local patches with simple food webs give rise to complex food webs at the metacommunity scale. Figure extracted from [157].

The dynamics of the model includes two processes: local extinction and colonization of adjacent patches. Every species $i$ goes locally (i.e. within each patch) extinct with a constant probability $e_{i}$. As a consequence of the extinction of species $i$ in a specific patch, all species directly or indirectly feeding upon species $i$ also go extinct. For example, in a patch with three species where species 3 feeds upon species 2 and species 2 feeds upon species 1 , species 1 goes extinct with probability $e_{1}$, species 2 goes extinct with probability $e_{1}+e_{2}$ and species 3 goes extinct with probability $e_{1}+e_{2}+e_{3}$.

Colonization allows a species $i$ that is established in a patch to establish itself in suitable patches with a constant rate $c_{i}$. A patch is considered suitable if a) it can be reached from the source patch, b) prey for the focal species is established in the patch and c) no superior competitor is already established in the patch.

Regarding a) we assume that the patches form a complex network. In this network every node represents a patch, and a link between two nodes indicates that colonization between the corresponding patches is possible.

Regarding the availability of suitable prey, b), we assume that only certain species, so-called basal species, can occupy empty patches. All other species have a set of at least one suitable prey species. A given species cannot colonize or persist in a patch unless at least one suitable prey is already established in the patch. These potential predator-prey relationships define a second network. In this network the nodes represent species, whereas directed links represent potential predator-prey interactions. This network is also the maximal food web that can be observed at the metacommunity scale. In any case, the emergent food web is a subgraph of the maximal food web, where potentially some nodes are missing, if species vanish. 
Regarding competition, c), following Pillai et al. [156, 157] we assume that specialists, which feed on one single prey species, are better adapted and therefore superior competitors to generalist, which are capable of feeding on several different prey species. If there is, for example, an exploitative competition between a specialist and a generalist in the same patch and the generalist cannot feed on the specialist then the generalist is quickly excluded. This is assumed to occur instantaneously in the model, so that a generalist cannot colonize a patch where a competing specialist is already established. Furthermore, an established generalist goes locally extinct if a competing specialist colonizes its patch. Note that, given this exclusion rule, the only possible food web configuration at the patch level is a linear chain (see Fig. 4.1).

\subsubsection{Persistence conditions for food chains}

We start our investigation by considering a case where the maximal food web is a simple food chain consisting of three trophic levels (see Fig. 4.2 a)). The main purpose of this simple example is to illustrate the calculation of coexistence ranges, which will be extended into a general rule for more complex cases in the subsequent section. A similar system, although on a different patch-network, was previously analyzed in [156]. Note that throughout this section the number of trophic levels $l$ is equivalent to the number of specialist species. In Section 4.3.1, when we consider multiple types of habitat, the number of species in general exceeds the number of trophic levels.

To gain a mathematical understanding of the metacommunity dynamics we first formulate a mean-field model describing the densities of specific patches. For this purpose we use a notation where symbols of the form $[i]$ indicate the proportion of patches where the local food chain has length $i$. Thus, [0], [1], [2] and [3] denote the fraction of empty patches, the fraction of patches exclusively inhabited by species 1, the proportion of patches inhabited by species 1 and species 2 , and the fraction of patches inhabited by all three species, respectively. Conservation of the number of patches then implies $[0]+[1]+[2]+[3]=1$. Additionally we use $p_{1}, p_{2}$ and $p_{3}$ to denote the proportion of patches where species 1 , species 2 and species 3 is present, irrespective of the presence of other species. Consequently, $p_{1}=[1]+[2]+[3], p_{2}=[2]+[3]$ and $p_{3}=[3]$.

The time evolution of the mean-field patch densities is then given by

$$
\begin{aligned}
\frac{d}{d t}[0] & =-c_{1}\langle k\rangle[0] p_{1}+e_{1} p_{1} \\
\frac{d}{d t}[1] & =c_{1}\langle k\rangle[0] p_{1}-c_{2}\langle k\rangle[1] p_{2}+e_{2} p_{2}-e_{1}[1], \\
\frac{d}{d t}[2] & =c_{2}\langle k\rangle[1] p_{2}-c_{3}\langle k\rangle[2] p_{3}+e_{3} p_{3}-\left(e_{1}+e_{2}\right)[2] \\
\frac{d}{d t}[3] & =c_{3}\langle k\rangle[2] p_{3}-\left(e_{1}+e_{2}+e_{3}\right)[3] .
\end{aligned}
$$

In these equations, the right-hand-side describes the effect of colonization and 


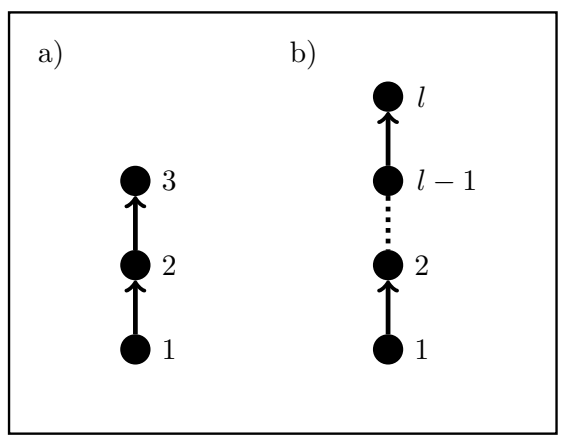

Figure 4.2: Schematic representation of food chains emerging at the metacommunity level. Nodes correspond to species, which are numbered according to their trophic levels. Arrows correspond to feeding links, pointing from prey species to predator species. a) Simple example system with three trophic levels. b) General food chain with $l$ trophic levels.

the effect of extinction. In the colonization terms, $\langle k\rangle$ denotes the mean degree of the patch-network. For now, we assume that the degree distribution of the patch-network is sufficiently narrow, so that it can be described to good approximation by a homogeneous approximation. In Section 4.3.2 we relax this assumption and derive corresponding equations for degree-heterogeneous patchnetworks.

Solving the system of equations (4.1) at equilibrium, yields the following equilibrium patch densities:

$$
\begin{aligned}
& {[0]^{*}=\frac{e_{1}}{c_{1}\langle k\rangle},} \\
& {[1]^{*}=\frac{e_{1}+e_{2}}{c_{2}\langle k\rangle}} \\
& {[2]^{*}=\frac{e_{1}+e_{2}+e_{3}}{c_{3}\langle k\rangle},} \\
& {[3]^{*}=1-\frac{e_{1}}{c_{1}\langle k\rangle}-\frac{e_{1}+e_{2}}{c_{2}\langle k\rangle}-\frac{e_{1}+e_{2}+e_{3}}{c_{3}\langle k\rangle}=1-\sum_{i=0}^{2}[i]^{*} .}
\end{aligned}
$$

Generalizing these results to systems where the maximal network is a linear food chain of length $l$ (see Fig. $4.2 \mathrm{~b}$ )), one finds

$$
\begin{aligned}
& {[i]^{*}=\frac{e_{1}+\cdots+e_{i+1}}{c_{i+1}\langle k\rangle}, \quad 0 \leq i<l} \\
& {[l]^{*}=1-\sum_{i=0}^{l-1}[i]^{*} .}
\end{aligned}
$$


To simplify the presentation we assume from now on that the extinction rates for all species are equal and the colonization rates for all species are equal, i.e. $e_{i}=e \forall i$ and $c_{i}=c \forall i$. Furthermore, we introduce the dimensionless parameters $z=e / c$ and $\bar{z}=z /\langle k\rangle$. In this notation the equilibrium patch densities read

$$
[i]^{*}=\left\{\begin{array}{lc}
(i+1) \bar{z}, & 0 \leq i<l \\
1-\frac{1}{2} i(i+1) \bar{z}, & i=l
\end{array}\right.
$$

Let us now investigate under which conditions a food chain of length $l$ can persist in the metacommunity. We use an approach motivated by epidemic literature $[58,59,131]$, where the stability of the disease-free equilibrium is checked against invasion of an epidemic. Here, we check the stability of the l-free equilibrium against arrival of species $l$. In other words, we perform linear stability analysis of a system where the maximal food web is given by a food chain of length $l-1$. If such a system is stable, species $l$ is not able to invade, while as soon as it becomes unstable species $l$ can establish itself in the metacommunity. Note that this concept is very similar to the percolation approach we introduced for the calculation of fragmentation thresholds in Section 3.2.

From the evolution equation for the patch density $[l]$,

$$
\frac{d}{d t}[l]=(c\langle k\rangle[l-1]-l e)[l],
$$

it follows that invasion of species $l$ is possible if

$$
c\langle k\rangle[l-1]^{*}-l e>0 .
$$

Here, $[l-1]^{*}$ denotes the equilibrium patch density of species $l-1$ in the $l$-free equilibrium, i.e. before arrival of species $l$. Employing (4.2) for a chain of length $l-1$, the invasion condition for $l$ can be rewritten as

$$
z<\frac{2\langle k\rangle}{l(l+1)}
$$

The inequality (4.3) represents a bound for the parameter $z$. We will refer to this type of bound as invasion threshold for species $l$. Invasion thresholds for specialists are important quantities for the analysis of metacommunities, as they determine the number of trophic levels which arise in the considered parameter range. Furthermore, persistence conditions in more complex food webs depend crucially on the invasion thresholds for specialists, as specialist species typically represent prey species for several predators. This issue will be discussed in more detail in the next section. 
From the obtained condition in (4.3), we deduce the following two implications: 1) The parameter $z$ determines the maximal length of the emergent chain of specialists. 2) Specialists at high trophic levels benefit from a high mean degree of the spatial patch-network. In other words, dense patch-networks promote a large number of species.

Note that at the point where species $l$ invades the system, at $\bar{z}=2 /(l(l+1))$, the equilibrium patch density $[l-1]^{*}$ switches from $p_{l-1}^{*}=1-\frac{1}{2} l(l-1) \bar{z}$ to $[l-1]^{*}=l \bar{z}$. So, the patch density $[l-1]^{*}$ increases linearly for $\bar{z}<2 /(l(l+1))$ and decreases linearly for $\bar{z}>2 /(l(l+1))$, until it vanishes at $\bar{z}=2 /(l(l-1))$. This discontinuity is also apparent in (4.2) and becomes important when we consider more complex food webs.

So far, we considered a very simple type of food web, namely a linear chain of specialists emerging in a metacommunity. For the calculation of the persistence condition for a chain of length $l$ we used a well-known approach from epidemics literature, which involves stability analysis of the $l$-free equilibrium. In the following section we extend this approach to more complex food webs.

\subsection{Influence of the food web on the persistence conditions}

In this section, we consider food webs consisting of specialist species and generalist species. In particular, we study the persistence conditions for omnivores, i.e. for generalists which feed on several trophic levels of the same food chain. Following the approach introduced in the last section, we calculate the parameter range where omnivores persist at the metacommunity scale. We provide a formalism which allows for a simple calculation and systematic analysis of persistence ranges. Such analysis gives insight into how the persistence condition depends on the specific structure of the food web.

First, we consider two simple examples where we illustrate the calculation of persistence conditions for specific omnivores in detail. Then, we generalize our analysis to omnivores feeding on two prey species at arbitrary trophic levels of a specialist chain. Finally, we extend the approach to omnivores feeding on more than two specialist prey species.

\subsubsection{Two simple food webs involving omnivores}

Our aim is to calculate the coexistence range, i.e. the parameter range where an omnivore coexists with a chain of specialists. In the following, we denote by omnivore a special type of generalist, which feeds exclusively on specialist prey species from a single food chain. In Section 4.3.1 we consider a broader class of generalists, which are capable of feeding in several independent food chains.

It is intuitive that an omnivore, in contrast to a specialist, faces two dangers. First, if the patch-network is very sparse the prey species may be too sparse to 
allow for invasion of the omnivore. Second, if the patch-network is too dense the specialist competing with the omnivore for the same prey will establish, which threatens the omnivore with competitive exclusion.

The first danger equally concerns specialists. Recall that scarceness of prey is the reason for the upper bound of $z$ in (4.3), beyond which invasion of specialists becomes impossible. The second danger, however, is peculiar to omnivores. As omnivores suffer competitive exclusion from specialists, there is also a lower bound for $z$, below which omnivores cannot stand the competition of the superior specialist. Thus, we expect the coexistence range for omnivores to be bounded by the invasion threshold from above and by the persistence threshold from below.

As a first example, we consider the food web configuration shown in Fig. 4.3. The maximal food web consists in this case of a linear food chain and an additional omnivore $x$ feeding on species 1 and species 2. We investigate the conditions under which the omnivore $x$ can coexist with the specialist food chain, although the specialist predator 3, if existent, will exclude the omnivore in any given patch.

We start by writing the governing equations for patches in which the omnivore $x$ is established as

$$
\begin{aligned}
& \frac{d}{d t}[1 x]=c\langle k\rangle\left([1] p_{x}-[1 x] p_{2}\right)+e([2 x]-2[1 x]), \\
& \frac{d}{d t}[2 x]=c\langle k\rangle\left([2] p_{x}+[1 x] p_{2}-[2 x] p_{3}\right)-3 e[2 x],
\end{aligned}
$$

where we used symbols of the form $[i x]$ to denote patches in which a specialist food chain of length $i$ and the omnivore $x$ is established. Note that omnivore $x$ does not suffer competitive exclusion in $[1 x]$-patches, because species 2 is not only a competitor for $x$, but also one of its prey species. So, colonization of species 2 turns a $[1 x]$-patch into a $[2 x]$-patch. This contribution is captured in the term $c\langle k\rangle[1 x] p_{2}$.

To allow for a more concise treatment we rewrite the equations (4.4) in matrix form,

$$
\frac{d}{d t}\left(\begin{array}{c}
{[1 x]} \\
{[2 x]}
\end{array}\right)=\underbrace{\left(\begin{array}{cc}
c\langle k\rangle\left([1]-p_{2}\right)-2 e & c\langle k\rangle[1]+e \\
c\langle k\rangle\left([2]+p_{2}\right) & c\langle k\rangle\left([2]-p_{3}\right)-3 e
\end{array}\right)}_{\mathbf{S}}\left(\begin{array}{c}
{[1 x]} \\
{[2 x]}
\end{array}\right),
$$

describing the time evolution of the vector $\vec{p}_{x}=([1 x],[2 x])$. We employ the same approach as in the case of the linear chain. There, we studied the stability of the $l$-free equilibrium against invasion of species $l$. Now, we analyze the stability of the $x$-free equilibrium against invasion of an omnivore $x$. This is done by analyzing the response of the $x$-free equilibrium to small perturbations. One can think of inserting a small fraction of patches containing species $x$ into 


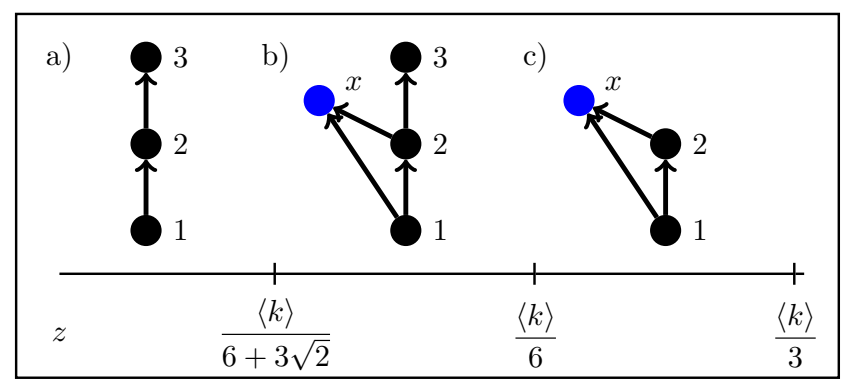

Figure 4.3: Examples of emergent food webs containing one omnivore. The maximal food web is the one depicted in b). Specialist species are depicted by black nodes, the omnivore is depicted by a blue node. In c), omnivore $x$ is able to invade a system of two specialists, in b) omnivore $x$ coexists with the specialist species 3 , and in a) omnivore $x$ becomes extinct due to competitive exclusion. The indicated parameter ranges corresponding to the different food web configurations are derived in the text.

the system. If $\lambda\left(\mathbf{S}^{*}\right)>0$, where $\mathbf{S}^{*}$ is the transition matrix in (4.5) evaluated at the $x$-free equilibrium and $\lambda$ is the largest eigenvalue of the matrix $\mathbf{S}^{*}$, a small fraction of $x$-patches suffices to establish omnivore $x$ in the system. So, from the condition $\lambda\left(\mathbf{S}^{*}\right)>0$ the corresponding threshold conditions for $z$ can be obtained. The goal is to extract an explicit condition for $z$ from the implicit condition $\lambda\left(\mathbf{S}^{*}\right)>0$ without solving the inequality numerically.

In the evaluation of the eigenvalue inequality, we have to take into account that $\mathbf{S}^{*}$ depends on the equilibrium patch density of species 3 . So, it makes a difference whether species 3 is present or absent in the system, when perturbing the system. Therefore, we have to analyze the stability of the $x$-free equilibrium separately for the two cases depicted in Fig. $4.3 \mathrm{~b}$ ) and c), corresponding to $p_{3}^{*} \neq 0$ and $p_{3}^{*}=0$. In order to distinguish between these two different cases, we introduce the following notation for the matrix $\mathbf{S}^{*}$, which governs the dynamics of $x$-patches close to the $x$-free equilibrium:

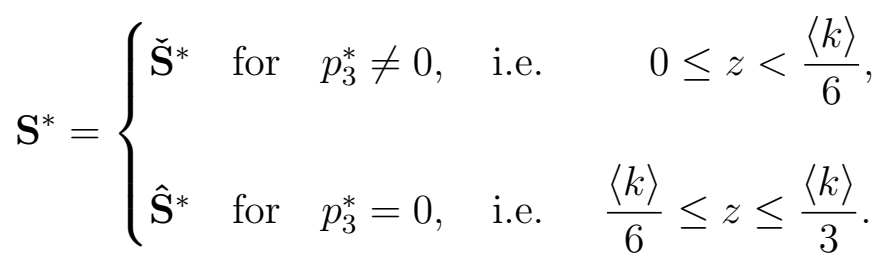

Here we used the result in (4.3) to obtain the invasion threshold for species 3. The discontinuity at $z=\langle k\rangle / 6$ is a resemblance of the switching from $[2]^{*}$ to $p_{2}^{*}$ as soon as species 3 dies out. Actually, (4.6) can be interpreted as the "omnivore version" of the equilibrium patch densities for specialists given in (4.2). This 
analogy becomes clear below, when we discuss the relationship between $\mathbf{S}^{*}$ and the equilibrium patch density of omnivores. Beyond $z=\langle k\rangle / 3$, one of the omnivore's prey species (species 2) does not exist, so that we do not have to consider that region of the parameter space.

Using the equilibrium expressions from (4.2) in the corresponding ranges and plugging them in the S-matrix given in (4.5) one obtains $\check{\mathbf{S}}^{*}$ and $\hat{\mathbf{S}}^{*}$. The coexistence range for the omnivore $x$ is then derived from a combination of the two persistence conditions, $\lambda\left(\check{\mathbf{S}}^{*}\right)>0$ and $\lambda\left(\hat{\mathbf{S}}^{*}\right)>0$. Rather than solving the eigenvalue condition numerically for both cases, we are looking for an explicit analytical expression for the coexistence range in terms of $z$. In the course of this section we develop a formalism for the calculation of persistence ranges, which directly yields conditions on $z$. We introduce the procedure using the simple example system depicted in Fig. 4.3, and later extend the approach to more general food webs.

We start by splitting the matrix $\mathbf{S}^{*}$ in the corresponding $z$-ranges indicated in (4.6) into a $z$-independent and a $z$-dependent part. In our example system, this reads

$$
\begin{aligned}
& \check{\mathbf{S}}^{*}=\check{\mathbf{s}}_{\mathbf{1}}+\bar{z} \check{\mathbf{S}}_{\mathbf{2}}=\left(\begin{array}{cc}
-1+3 \bar{z} & 3 \bar{z} \\
1 & -1+6 \bar{z}
\end{array}\right)=\underbrace{\left(\begin{array}{cc}
-1 & 0 \\
1 & -1
\end{array}\right)}_{\check{\mathbf{s}}_{\mathbf{1}}}+\underbrace{\left(\begin{array}{ll}
3 & 3 \\
0 & 6
\end{array}\right)}_{\check{\mathbf{s}}_{\mathbf{2}}}, \\
& \hat{\mathbf{S}}^{*}=\hat{\mathbf{s}}_{\mathbf{1}}+\bar{z} \hat{\mathbf{s}}_{\mathbf{2}}=\left(\begin{array}{cc}
-1+3 \bar{z} & 3 \bar{z} \\
2-6 \bar{z} & 1-6 \bar{z}
\end{array}\right)=\underbrace{\left(\begin{array}{cc}
-1 & 0 \\
2 & 1
\end{array}\right)}_{\hat{\mathbf{s}}_{\mathbf{1}}}+\bar{z} \underbrace{\left(\begin{array}{cc}
3 & 3 \\
-6 & -6
\end{array}\right)}_{\hat{\mathbf{s}}_{\mathbf{2}}} .
\end{aligned}
$$

Such a splitting is always possible for $\mathbf{S}^{*}$-matrices which contain linear entries in $z$. In particular, for predators feeding on specialist prey $\mathbf{S}^{*}$ is linear in $z$, according to (4.2).

Now, we observe the following: at the left boundary of the validity range of $\check{\mathbf{S}}^{*}$, at $z=0$, the condition $\lambda\left(\check{\mathbf{S}}^{*}\right)>0$ is obviously not fulfilled, as $\check{\mathbf{s}}_{1}^{*}$ has only negative eigenvalues. Consequently, $\operatorname{det}\left(\check{\mathbf{S}}^{*}\right)>0$ at $z=0$. For increasing $z$, at some point $\operatorname{det}\left(\check{\mathbf{S}}^{*}\right)$ becomes negative, which implies that the largest eigenvalue of $\check{\mathbf{S}}^{*}$ becomes positive. The first $z$-value where $\operatorname{det}\left(\check{\mathbf{S}}^{*}\right)=0$ thus represents the lower bound of the persistence range of omnivore $x$.

In order to express the condition $\operatorname{det}\left(\check{\mathbf{S}}^{*}\right)<0$ in terms of $z$, we make use of

$$
\operatorname{det}\left(\mathbf{S}^{*}\right)=\operatorname{det}(\bar{z} \mathbf{M}-\mathbf{1}) \cdot \operatorname{det}\left(-\mathbf{S}_{\mathbf{1}}\right) \text {. }
$$

Here, we introduced the iteration matrix $\mathbf{M}=-\mathbf{S}_{\mathbf{2}} \mathbf{s}_{\mathbf{1}}{ }^{-1}$, which is given by

$$
\check{\mathbf{M}}=-\check{\mathbf{s}}_{2} \check{\mathbf{s}}_{1}^{-1} \quad \text { and } \quad \hat{\mathbf{M}}=-\hat{\mathbf{s}}_{2} \hat{\mathbf{s}}_{1}^{-1},
$$

in the respective intervals defined in (4.6). In epidemics literature, matrices of this kind are often referred to as next generation matrices, and their largest 
eigenvalues are related to the basic reproduction number $[59,131]$. The interpretation in our ecological model is very similar: The matrix $\mathbf{M}$ describes the growth or decrease in the number of $x$-patches, based on the number of $x$-patches at a previous time-step, i.e. the average reproduction of species $x$. From a mathematical point of view, one can associate $\mathbf{M}$ with a discrete-time version of (4.5). At $\mathbf{S}^{*} \vec{p}_{x}=0$, we have $\vec{p}_{x}=\bar{z} \mathbf{M} \vec{p}_{x}$, which implies that $\lambda(\mathbf{M})$ is related to the stability of the $x$-free equilibrium ${ }^{8}$. In the following we argue that the bounds of the persistence range can be expressed in terms of $\lambda(\mathbf{M})$.

We start with the lower bound. Recall that the stability of the $x$-free equilibrium changes when $\operatorname{det}\left(\check{\mathbf{S}}^{*}\right)$ changes sign. Given that $\operatorname{det}\left(-\check{\mathbf{S}}_{1}^{*}\right)>0$, the condition $\operatorname{det}\left(\check{\mathbf{S}}^{*}\right)<0$ is, according to $(4.7)$, equivalent to $\operatorname{det}(\bar{z} \mathbf{M}-\mathbf{1})<0$. Because a change in stability of the continuous-time system implies a change in stability of the discrete-time system, we are only interested in the case where the sign change of $\operatorname{det}(\bar{z} \check{M}-\mathbf{1})$ is caused by $\bar{z} \lambda(\mathbf{M})$ crossing 1 . This leads to

$$
\lambda\left(\check{\mathbf{S}}^{*}\right)>0 \hat{=} \lambda(\check{\mathbf{M}})>\frac{1}{\bar{z}} .
$$

This equivalence provides a simple tool for the calculation of persistence thresholds, through calculation of the largest eigenvalue of a constant matrix $\check{\text { M. }}$

In our example system, we have

$$
\check{\mathbf{M}}=\underbrace{\left(\begin{array}{ll}
3 & 3 \\
0 & 6
\end{array}\right)}_{\check{\mathbf{s}}_{\mathbf{2}}} \cdot \underbrace{\left(\begin{array}{ll}
1 & 0 \\
1 & 1
\end{array}\right)}_{-\check{\mathbf{s}}_{1}^{-1}}=\left(\begin{array}{ll}
6 & 3 \\
6 & 6
\end{array}\right) .
$$

With (4.8) we obtain a lower bound for the parameter $z$, the persistence threshold,

$$
z>\frac{\langle k\rangle}{\lambda(\check{\mathbf{M}})}=\frac{\langle k\rangle}{6+3 \sqrt{2}} .
$$

This lower bound limits the persistence of $x$ due to competition with the specialist 3 .

In principle, there could be a $z$-value in the validity range of $\check{\mathbf{S}}^{*}$, where $\lambda\left(\check{\mathbf{S}}^{*}\right)$ becomes negative again. However, we have to take into account that the patch density $p_{3}^{*}$ decreases linearly with increasing $z$, while at the same time the abundance of available prey patches, $[1]^{*}$ and $[2]^{*}$ increases. So, ecological reasoning suggests that the omnivore, once it is established in the system, does not die out before $[1]^{*}$ and $[2]^{*}$ start to decrease at $\bar{z}=1 / 6$. The omnivore does, however, die out at some point $\bar{z}^{\prime}$ in the validity range of $\hat{\mathbf{S}}^{*}$, due to sparseness of its prey. The $z$-value $z^{\prime}$, where this occurs can be calculated in a similar way to the calculation of the persistence threshold.

${ }^{8}$ Here, we used that the M-matrices in our model have only positive eigenvalues. In general, $\rho(\mathbf{M})$, the largest absolute eigenvalue of $\mathbf{M}$, is associated with the stability of the system. 
For the upper bound, we start by considering the right boundary of the validity range of $\hat{\mathbf{S}}^{*}$. It is straight-forward to verify that the condition $\lambda\left(\hat{\mathbf{S}}^{*}\right)>0$ is not met, i.e. the omnivore cannot survive at $\bar{z}=1 / 3$. One can intuitively understand this result, as the omnivore $x$ can only survive when both of its prey species are available, but species 2 dies out at $\bar{z}=1 / 3$. Again, we look for the first $z$-value where $\operatorname{det}\left(\hat{\mathbf{S}}^{*}\right)$ becomes negative, now starting at $\bar{z}=1 / 3$ and going in decreasing $z$-direction. The condition $\operatorname{det}\left(\hat{\mathbf{S}}^{*}\right)<0$ is now equivalent to $\operatorname{det}(\bar{z} \hat{\mathbf{M}}-\mathbf{1})>0$, due to $(4.7)$ and because $\operatorname{det}\left(-\hat{\mathbf{s}_{\mathbf{1}}}\right)<0$. This time $\bar{z} \lambda(\hat{\mathbf{M}})$ crosses 1, coming from above. Therefore, the condition in terms of $z$ yields $\bar{z} \lambda(\hat{\mathbf{M}})<1$. This leads to the following equivalence for the upper bound of the coexistence range:

$$
\lambda\left(\hat{\mathbf{S}}^{*}\right)>0 \hat{=} \quad \lambda(\hat{\mathbf{M}})<\frac{1}{\bar{z}}
$$

In our example, we have

$$
\hat{\mathbf{M}}=\underbrace{\left(\begin{array}{cc}
3 & 3 \\
-6 & -6
\end{array}\right)}_{\hat{\mathbf{s}}_{\mathbf{2}}} \cdot \underbrace{\left(\begin{array}{cc}
1 & 0 \\
-2 & -1
\end{array}\right)}_{-\hat{\mathbf{s}}_{\mathbf{1}}^{-1}}=\left(\begin{array}{cc}
-3 & -3 \\
6 & 6
\end{array}\right) .
$$

Therefore, the upper bound for $z$, the invasion threshold, yields

$$
z<\frac{\langle k\rangle}{\lambda(\hat{\mathbf{M}})}=\frac{\langle k\rangle}{3} .
$$

Note that this is at the same time the invasion condition for species 2 (according to $(4.3)$, with $l=2$ ). This means, as soon as a chain of two specialists exists in the metacommunity, an omnivore feeding upon both of them can invade the system. Later, we will see that this holds for any omnivore feeding upon two prey species at subsequent trophic levels, and more generally for omnivores with $n$ prey species where the highest two prey species are at subsequent trophic levels.

Combining conditions (4.9) and (4.11), we conclude that for

$$
\frac{\langle k\rangle}{6+3 \sqrt{2}}<z<\frac{\langle k\rangle}{3}
$$

omnivore $x$ is able to coexist with a chain of specialists at the metacommunity level. In contrast to the result which was obtained in [157] by numerically solving the system of equations in (4.4), here we calculated the threshold conditions for $z$ directly by determining the largest eigenvalues of the respective next generation matrices. Furthermore, we derived an upper bound for the coexistence range of the omnivore $x$, which goes beyond the persistence range of species 3, revealing that an omnivore feeding on species 1 and species 2 can survive in a $z$-range where species 3 dies out. The approach we presented here 


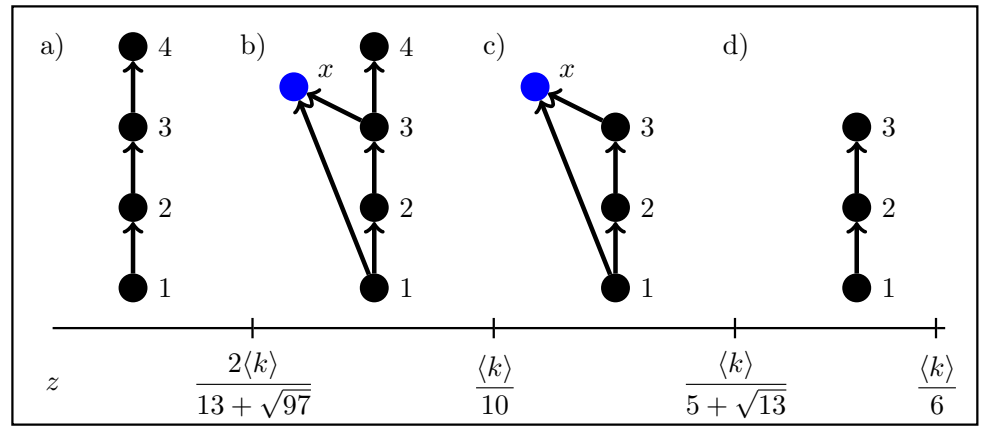

Figure 4.4: Examples of emergent food webs containing one omnivore. The maximal food web is the one depicted in b). In contrast to Fig. 4.3, the trophic levels of the omnivore's prey species differ by more than one. The indicated parameter ranges corresponding to the different food web configurations are obtained with the mathematical procedure described in the text.

allows for a generalization and simple analysis of a broad class of food webs in metacommunities, as we will see in the following sections.

Let us summarize the obtained results for the example system shown in Fig. 4.3. For $z<\langle k\rangle / 3$ both, specialist species 2 , and omnivore species $x$ can invade the system (c). While the existence range for species 2 is not bounded from below, the existence range for omnivore $x$ is bounded, due to competition with specialist 3, which can invade the system for $z<\langle k\rangle / 6$. Omnivore $x$ can coexist with the superior competitor 3 for $z>\langle k\rangle /(6+3 \sqrt{2})$ (b). For $z<\langle k\rangle /(6+3 \sqrt{2})$ the omnivore becomes extinct and the resulting food web is a linear chain of three specialists (a).

Before we generalize our results, let us consider a second example where an omnivore feeds on two prey species which are not at subsequent trophic levels (see Fig. 4.4). For this example, the evolution equation for $\vec{p}_{x}$ is given by

$$
\frac{d}{d t}\left(\begin{array}{c}
{[1 x]} \\
{[3 x]}
\end{array}\right)=\left(\begin{array}{cc}
c\langle k\rangle\left([1]-p_{2}\right)-2 e & c\langle k\rangle[1]+e \\
c\langle k\rangle[3] & c\langle k\rangle\left([3]-p_{4}\right)-4 e
\end{array}\right)\left(\begin{array}{l}
{[1 x]} \\
{[3 x]}
\end{array}\right) .
$$

In contrast to the previous example, now omnivore $x$ suffers competitive exclusion, both in [1x]- and in [3x]-patches, because none of its competitors (species 2 and species 4) coincides with one of its prey species. Consequently, the structure of the S-matrix differs from the one given in (4.5) in the entry $S_{21}$. We will see, that this little distinction induces no major qualitative difference in the results for the coexistence ranges. However, it cannot be neglected and we will always have to distinguish whether prey species are at subsequent trophic levels or not. 


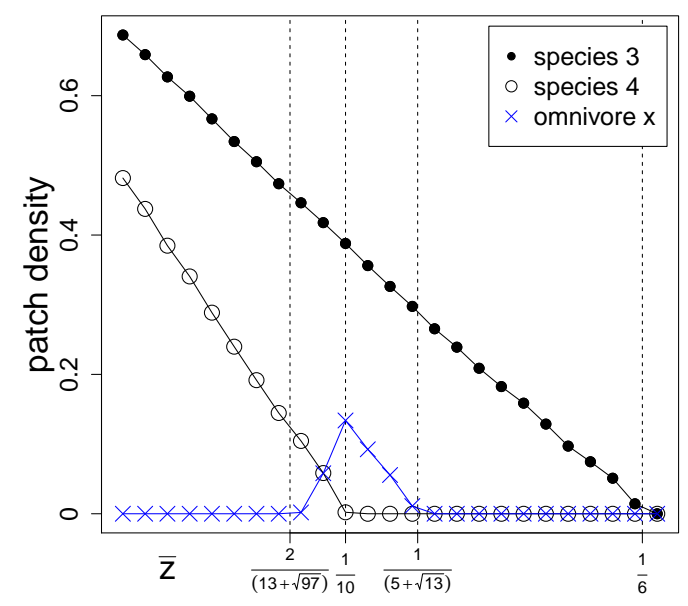

Figure 4.5: Comparison of the predicted persistence ranges with simulation results. Plotted are the equilibrium patch densities $p_{3}^{*}(\bullet), p_{4}^{*}(\circ)$ and $p_{x}^{*}(\times)$ obtained from network simulations. Each point corresponds to an average over 10 independent simulation runs. We used $N=10000,\langle k\rangle=20, c=0.05$ and varied $\bar{z}=e$ within the indicated range. Dashed lines correspond to the thresholds predicted by our analytical calculations. Coexistence range for omnivore $x: 2 /(13+\sqrt{97})<\bar{z}<1 /(5+\sqrt{13})$, invasion threshold for specialist $3: \bar{z}=1 / 6$ and invasion threshold for specialist $4: \bar{z}=1 / 10$.

We follow the same procedure as before. First, we construct $\check{\mathbf{S}}^{*}$ and $\hat{\mathbf{S}}^{*}$ at $x$-free equilibrium. For $p_{4}^{*} \neq 0$ we obtain with (4.2),

$$
\check{\mathbf{S}}^{*}=\left(\begin{array}{cc}
-1+3 \bar{z} & 3 \bar{z} \\
4 \bar{z} & -1+10 \bar{z}
\end{array}\right)=\underbrace{-\left(\begin{array}{cc}
1 & 0 \\
0 & 1
\end{array}\right)}_{\widetilde{\mathbf{s}}_{1}}+\underbrace{\left(\begin{array}{cc}
3 & 3 \\
4 & 10
\end{array}\right)}_{\widetilde{\mathbf{s}}_{2}} .
$$

For $p_{4}^{*}=0$, we have

$$
\hat{\mathbf{S}}^{*}=\left(\begin{array}{cc}
-1+3 \bar{z} & 3 \bar{z} \\
1-6 \bar{z} & 1-10 \bar{z}
\end{array}\right)=\underbrace{\left(\begin{array}{cc}
-1 & 0 \\
1 & 1
\end{array}\right)}_{\mathbf{s}_{1}}+\underbrace{\left(\begin{array}{cc}
3 & 3 \\
-6 & -10
\end{array}\right)}_{\mathbf{s}_{2}} .
$$

Consequently, the related next generation matrices $\check{\mathbf{M}}$ and $\hat{\mathbf{M}}$ read

$$
\check{\mathbf{M}}=\underbrace{\left(\begin{array}{cc}
3 & 3 \\
4 & 10
\end{array}\right)}_{\check{\mathbf{s}}_{2}} \cdot \underbrace{\left(\begin{array}{ll}
1 & 0 \\
0 & 1
\end{array}\right)}_{-\widetilde{\mathbf{s}}_{\mathbf{1}}^{-1}}=\left(\begin{array}{cc}
3 & 3 \\
4 & 10
\end{array}\right),
$$


and

$$
\hat{\mathbf{M}}=\underbrace{\left(\begin{array}{cc}
3 & 3 \\
-6 & -10
\end{array}\right)}_{\mathbf{s}_{\mathbf{2}}} \cdot \underbrace{\left(\begin{array}{cc}
1 & 0 \\
-1 & -1
\end{array}\right)}_{-\mathbf{s}_{\mathbf{1}}-1}=\left(\begin{array}{cc}
0 & -3 \\
4 & 10
\end{array}\right) .
$$

An analogous argumentation as in the previous example leads to the equivalences (4.8) and (4.10), and accordingly to the following coexistence threshold:

$$
\frac{2\langle k\rangle}{13+\sqrt{97}}<z<\frac{\langle k\rangle}{5+\sqrt{13}} .
$$

Comparing this range to the one given in (4.12), we observe that the whole range is shifted to smaller values of $z$, and that the total size of the coexistence range is smaller compared to the previous example. These two general features originate in the different sets of prey species of the two different omnivores. In particular, a higher trophic level of the prey species and a larger distance between the two prey species (in terms of trophic level) lead to a smaller coexistence range. We show the general validity of this statement below.

Note that the invasion threshold in this example does not coincide with the invasion threshold for species 3 (which is $z<\langle k\rangle / 6$, according to (4.3)). So, in contrast to the previous example where the two prey species were at subsequent trophic levels, now the presence of both prey species (species 1 and species 3 ) in the system does not imply that invasion of species $x$ is possible. Fig. 4.4 shows a summary of the obtained results for this second example system. In Fig. 4.5, simulation results for the same system are plotted. It can be seen that the analytically estimated ranges correspond to disappearance or appearance of the respective species types, indicating that the predicted food web configurations are actually realized in the metacommunity.

In this section, we demonstrated for two simple example systems how the coexistence range of an omnivore can be directly calculated via the largest eigenvalue of an associated next generation matrix $\mathbf{M}$. The considered examples already indicate a dependence of the coexistence range on the trophic levels of the prey species. In the following section we apply the proposed approach to more general cases, allowing for a quantitative analysis of the impact of the prey species on the coexistence conditions.

\subsubsection{Omnivores feeding on two arbitrary prey species}

In the following, we consider omnivores which feed on specialist prey species at arbitrary trophic levels. For now, we restrict our analysis to omnivores feeding on two prey species. In the next section, we discuss the case of more than two prey species. We denote the trophic levels of the omnivore's prey by $i-1$ and $j-1$, where we assume $i>j$ if not otherwise stated. This implies that the 


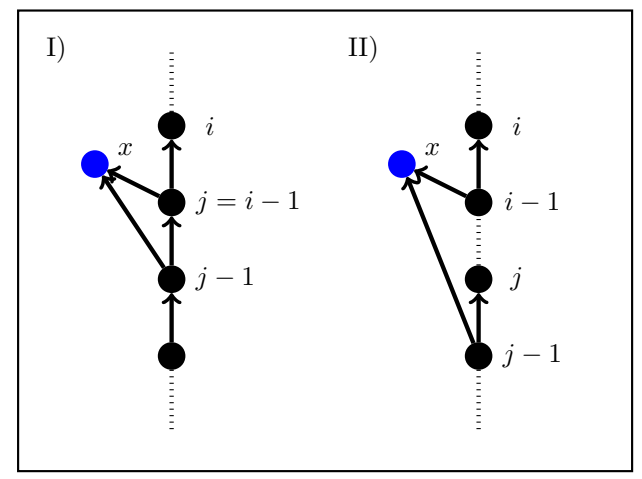

Figure 4.6: Food web configuration consisting of a chain of specialists and one omnivore. I) The omnivore $x$ feeds on two subsequent trophic levels, i.e. $i=j+1$. II) The omnivore $x$ feeds on two non-subsequent trophic levels, i.e. $i>j+1$. Accordingly, different $\mathbf{S}^{*}$-matrices are obtained for both cases, as described in the text.

specialists competing with the respective omnivore are the ones at trophic levels $i$ and $j$. Further, we refer to species $i$ by principal competitor and to species $i-1$ by principal prey.

It is clear from the examples considered in the last section that we have to distinguish between two different cases (see Fig. 4.6): I) The omnivore feeds upon prey at subsequent trophic levels $(i=j+1)$. II) The omnivore feeds upon prey at non-subsequent trophic levels $(i>j+1)$. The $\mathbf{S}^{*}$-matrices governing the evolution equations for $[(i-1) x]$ - and $[(j-1) x]$-patches in the $x$-free equilibrium are accordingly given by

$$
\mathbf{S}_{\mathrm{I}}^{*}=\left(\begin{array}{cc}
{[j-1]^{*}-p_{j}^{*}-j \bar{z}} & {[j-1]^{*}+\bar{z}} \\
{[i-1]^{*}+p_{i-1}^{*}} & {[i-1]^{*}-p_{i}^{*}-i \bar{z}}
\end{array}\right),
$$

and

$$
\mathbf{S}_{\mathrm{II}}^{*}=\left(\begin{array}{cc}
{[j-1]^{*}-p_{j}^{*}-j \bar{z}} & {[j-1]^{*}+\bar{z}} \\
{[i-1]^{*}} & {[i-1]^{*}-p_{i}^{*}-i \bar{z}}
\end{array}\right)
$$

where we introduced the subscripts I and II in order to distinguish between the two different cases. Note that $\mathbf{S}_{\mathrm{I}}^{*}$ yields for $j-1=1$ and $i-1=2$ the transition matrix of our first example in (4.5), while $\mathbf{S}_{\mathrm{II}}^{*}$ reproduces for $j-1=1$ and $i-1=3$ the transition matrix of our second example in (4.13). Because the structure of the general transition matrices in (4.14) and (4.15) is obviously the same as in our two previous examples, we can employ the same procedure as 
before to calculate the persistence range of an omnivore $x$ feeding on arbitrary trophic levels.

First, we split the parameter range according to presence or absence of the principal competitor $i$. As invasion of species $i$ becomes possible for $\bar{z}<2 /(i(i+$ 1)) and the principal prey species $i-1$ dies out for $\bar{z}>2 /(i(i-1))$, we obtain the following validity ranges for the matrices $\check{\mathbf{S}}^{*}$ and $\hat{\mathbf{S}}^{*}$ :

$$
\mathbf{S}^{*}=\left\{\begin{array}{lrr}
\check{\mathbf{S}}^{*}=\check{\mathbf{s}}_{\mathbf{1}}+\bar{z} \check{\mathbf{s}}_{\mathbf{2}}, & \text { for } & 0 \leq z<\frac{2\langle k\rangle}{i(i+1)}, \\
\hat{\mathbf{S}}^{*}=\hat{\mathbf{s}}_{\mathbf{1}}+\bar{z} \hat{\mathbf{s}}_{\mathbf{2}}, & \text { for } & \frac{2\langle k\rangle}{i(i+1)} \leq z \leq \frac{2\langle k\rangle}{i(i-1)}
\end{array}\right.
$$

This splitting applies equally to $\mathbf{S}_{\mathrm{I}}^{*}$ and $\mathbf{S}_{\mathrm{II}}^{*}$.

Then, we follow the same argumentation as before. $\mathbf{S}^{*}$ is stable at both boundaries of the validity range, i.e. $\lambda\left(\check{\mathbf{S}}^{*}\right)<0$ at $z=0$ and $\lambda\left(\hat{\mathbf{S}}^{*}\right) \leq 0{ }^{9}$ at $\bar{z}=2 /(i(i-1))$. Approaching $\bar{z}=2 /(i(i+1))$ from both boundaries, $\mathbf{S}^{*}$ becomes unstable at two $z$-values, namely when $\lambda\left(\check{\mathbf{S}}^{*}\right)>0$ and when $\lambda\left(\hat{\mathbf{S}}^{*}\right)>0$. A change in stability of $\mathbf{S}^{*}$ implies a change in stability of the associated discrete-time system, described by the iteration matrix $\mathbf{M}$. In the range $0 \leq \bar{z}<2 /(i(i+1))$, $\lambda(\bar{z} \check{\mathbf{M}})$ becomes larger than 1 when $\check{\mathbf{S}}^{*}$ becomes unstable, while in the range $2 /(i(i+1)) \leq \bar{z} \leq 2 /(i(i-1)), \lambda(\bar{z} \check{\mathbf{M}})$ becomes smaller than 1 when $\hat{\mathbf{S}}^{*}$ becomes unstable. This is due to $\operatorname{det}\left(-\check{\mathbf{s}}_{\mathbf{1}}\right)>0$, but $\operatorname{det}\left(-\hat{\mathbf{s}}_{\mathbf{1}}\right)<0$, taking relation (4.7) into account. Consequently, the coexistence range for an omnivore feeding on two arbitrary trophic levels is for the two different cases given by

$$
\frac{\langle k\rangle}{\lambda\left(\check{\mathbf{M}}_{\mathrm{I}}\right)}<z<\frac{\langle k\rangle}{\lambda\left(\hat{\mathbf{M}}_{\mathrm{I}}\right)},
$$

$$
\frac{\langle k\rangle}{\lambda\left(\check{\mathbf{M}}_{\mathrm{II}}\right)}<z<\frac{\langle k\rangle}{\lambda\left(\hat{\mathbf{M}}_{\mathrm{II}}\right)}
$$

Therefore, the coexistence range can be directly calculated via the respective iteration matrix $\mathbf{M}$. The corresponding $\mathbf{M}$-matrices are defined as previously $\left(\mathbf{M}=-\mathbf{s}_{\mathbf{2}} \mathbf{s}_{\mathbf{1}}{ }^{-1}\right)$ and can be obtained from the general expressions for the

\footnotetext{
${ }^{9}$ Equality holds for $i=j+1$, implying that the upper bound of the coexistence range coincides with the invasion threshold of species $i-1$.
} 
$\mathbf{S}^{*}$-matrices in (4.14) and (4.15):

$$
\begin{array}{ll}
\check{\mathbf{M}}_{\mathrm{I}}=\left(\begin{array}{cc}
\frac{i(i+1)}{2} & i \\
2 i & \frac{i(i+1)}{2}
\end{array}\right), & \hat{\mathbf{M}}_{\mathrm{I}}=\left(\begin{array}{cc}
\frac{i(i-5)}{2} & -i \\
2 i & \frac{i(i+1)}{2}
\end{array}\right), \\
\check{\mathbf{M}}_{\mathrm{II}}=\left(\begin{array}{cc}
\frac{j(j+1)}{2} & j+1 \\
i & \frac{i(i+1)}{2}
\end{array}\right), & \hat{\mathbf{M}}_{\mathrm{II}}=\left(\begin{array}{cc}
\frac{(j-2)(j+1)}{2} & -(j+1) \\
i & \frac{i(i+1)}{2}
\end{array}\right) .
\end{array}
$$

Relation (4.17), together with the matrices given in (4.18), provide a general expression for the coexistence range of any omnivore feeding on two prey species of a specialist chain. These general formulae allow for an analysis of the dependence of the coexistence range on the trophic levels of the prey species, $i-1$ and $j-1$, revealing the following two results:

- The coexistence range for an omnivore with two prey species becomes smaller with increasing trophic level of the prey species.

- The coexistence range for an omnivore with two prey species becomes smaller with increasing distance between the trophic levels of its prey species.

These characteristics of the coexistence range are shown in Fig. 4.7. The figure also reveals the parameter range where most omnivores are able to survive (dashed region). The maximal number of omnivores existing in that range is given by $n_{\max }=i-1$. The dashed lines correspond to the invasion thresholds of the principal competitors $(z=2\langle k\rangle /(i(i+1)))$ where the discontinuity in $\mathbf{S}^{*}$ occurs.

From the formulae in (4.17) the impact of the connectivity of the patchnetwork becomes clear: dense topologies promote the persistence of omnivores in the metacommunity, as the coexistence range increases for increasing mean degree $\langle k\rangle$. Roughly speaking, dense patch-networks convey species richness, an observation which is consistent with what we found in the case of food chains.

As a final remark in this section, we emphasize that the coexistence range of an omnivore only depends on the trophic levels of its prey species, and not on the total length of the specialist chain. This is a consequence of the following relationship,

$$
[i-1]+[(i-1) x]= \begin{cases}i \bar{z}, & p_{i} \neq 0 \\ 1-\frac{1}{2} i(i-1) \bar{z}, & p_{i}=0\end{cases}
$$

which can be obtained by adding the evolution equations for $[i-1]$-patches and $[(i-1) x]$-patches and solving at equilibrium. Note that (4.19) describes the 


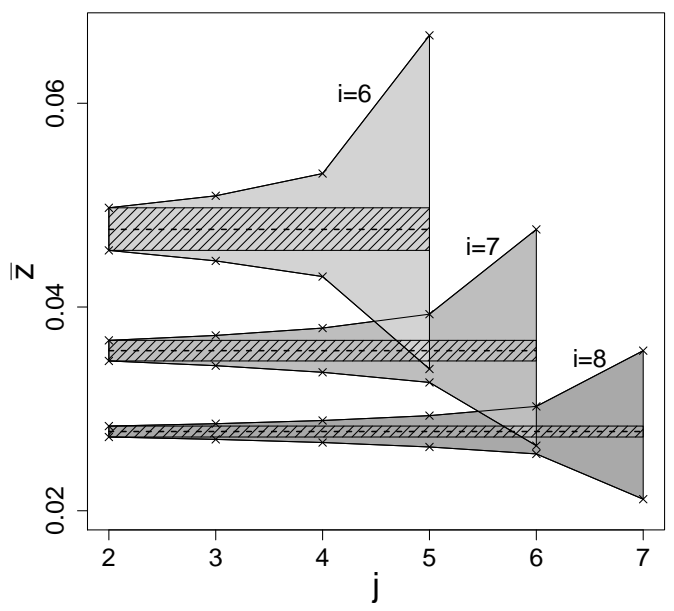

Figure 4.7: Coexistence range for an omnivore in dependence on the trophic levels of its prey species. The grey regions show the coexistence ranges for different values of $i$ and varying $j$, corresponding to the formulae given in (4.17). The coexistence range becomes smaller with increasing $i$ and with decreasing $j$ (for fixed $i$ ). Dashed lines indicate the respective invasion thresholds of species $i$. The dashed region marks the parameter range where most omnivores are able to coexist with the specialist chain. The maximal number of coexisting omnivores for a given chain of length $i$ yields $n_{\max }=i-1$.

equilibrium patch density of $[i-1]$ in general, not necessarily at $x$-free equilibrium. At $x$-free equilibrium, i.e. for $[(i-1) x]=0$, the equilibrium densities given in (4.2) are recovered and $[i-1]$ becomes $[i-1]^{*}$. Relation (4.19) can be interpreted as "biomass-conservation", implying that the species densities $p_{i}$ are conserved, no matter how many predators feed upon species $i$, and upon prey at lower trophic levels.

With (4.19) we can obtain a simple expression for the equilibrium patch density $p_{x}^{*}$ within the persistence range of omnivore $x$. To show this, let us recall the evolution equation for the patch density $p_{x}$,

$$
\frac{d}{d t} \vec{p}_{x}=\mathbf{S} \vec{p}_{x}
$$

where $\mathbf{S}$ denotes the transition matrix for $x$-patches. For example, in case II $(i>j+1)$, the transition matrix is given by

$$
\mathbf{S}=\left(\begin{array}{cc}
{[j-1]-p_{j}-j \bar{z}} & {[j-1]+\bar{z}} \\
{[i-1]} & {[i-1]-p_{i}-i \bar{z}}
\end{array}\right)
$$


In contrast to the $\mathbf{S}^{*}$-matrix in (4.15), which describes the $x$-free equilibrium, the matrix $\mathbf{S}$ here is in general valid, i.e. also when $x$ is established in the system. In this case, the amount of suitable resource patches for omnivore $x$ is reduced, because patches where the omnivore is already established are not available. More precisely, $[i-1]=[i-1]^{*}-[(i-1) x]$ and $[j-1]=[j-1]^{*}-[(j-1) x]$. Accordingly, one can relate the general transition matrix $\mathbf{S}$ to the corresponding matrix $\mathbf{S}^{*}$ at $x$-free equilibrium:

$$
\mathbf{S}=\mathbf{S}^{*}-\left(\begin{array}{ll}
{[(j-1) x]} & {[(j-1) x]} \\
{[(i-1) x]} & {[(i-1) x]}
\end{array}\right)
$$

Rewriting (4.20), reveals

$$
\frac{d}{d t} \vec{p}_{x}=\left\{\mathbf{S}^{*}-\left(\begin{array}{ll}
{[(j-1) x]} & {[(j-1) x]} \\
{[(i-1) x]} & {[(i-1) x]}
\end{array}\right)\right\} \vec{p}_{x}=\left(\mathbf{S}^{*}-p_{x}\right) \vec{p}_{x} .
$$

So, the equilibrium patch density $p_{x}^{*}$ is given by the largest eigenvalue of the corresponding transition matrix $\mathbf{S}^{*}$ at $x$-free equilibrium:

$$
p_{x}^{*}=[(j-1) x]^{*}+[(i-1) x]^{*}=\lambda\left(\mathbf{S}^{*}\right) .
$$

The discontinuity of $\mathbf{S}^{*}$ (see (4.16)) is inherited by $p_{x}^{*}$, leading to an increasing patch density before the invasion of species $i(\bar{z}>2 /(i(i+1)))$ and a decreasing patch density after the invasion of the principal competitor $(\bar{z}<2 /(i(i+1)))$. Both, increase and decrease proceed almost linearly ${ }^{10}$, as Fig. 4.8 demonstrates. This observation supports our previous reasoning, where we claimed that $\mathbf{S}^{*}$ changes stability only once in the range $0 \leq \bar{z}<2 /(i(i+1))$, and once in the range $2 /(i(i+1)) \leq \bar{z} \leq 2 /(i(i-1))$.

To summarize, in this section we extended our approach for the calculation of persistence ranges to omnivores feeding on two prey species at arbitrary trophic levels. The analysis of the obtained persistence ranges reveals how the trophic levels of the prey species influence the extension of the coexistence range of the omnivore. In particular, we found that omnivores feeding on prey species at similar trophic levels are able to exist in a larger region of the parameter space than omnivores feeding on prey species at rather differing trophic levels. Furthermore, we derived a simple expression for the equilibrium patch density of omnivores, in terms of the largest eigenvalue of the transition matrix $\mathbf{S}^{*}$.

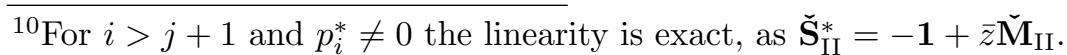




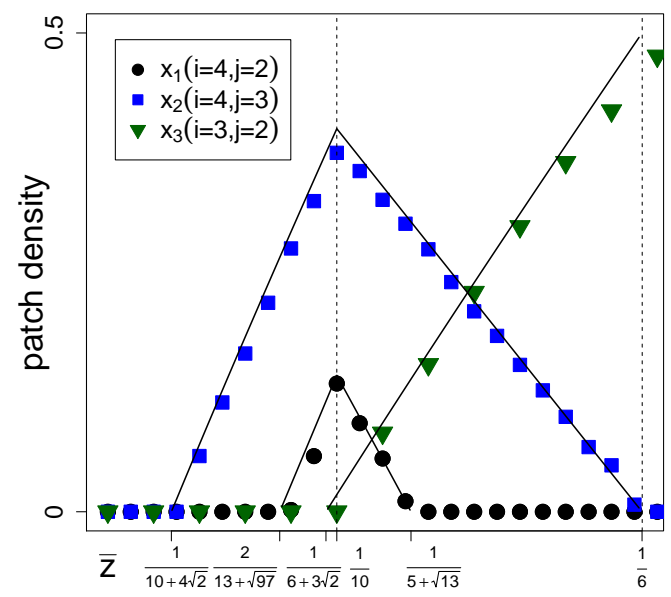

Figure 4.8: Equilibrium patch densities of omnivores. Symbols correspond to simulation results for three different omnivores. Solid lines correspond to theoretical predictions. Dashed lines indicate parameter values where the respective principal competitor goes extinct. The equilibrium patch densities observed in simulations display an almost linear behavior and are well described by $p_{x}^{*}=\lambda\left(\mathbf{S}^{*}\right)$. The simulation parameters are the same as in Fig. 4.5.

\subsubsection{Omnivores feeding on more than two prey species}

In this section, we address a situation where an omnivore is capable of feeding on $n$ different specialist prey species. Again, we refer to the highest prey (at trophic level $i-1$ ) by principal prey, and to the highest competitor (at trophic level $i$ ) by principal competitor. Given a set of prey species, the structure of the $\mathbf{S}^{*}$-matrix depends on the trophic level of all prey species and on the distance between consecutive prey species. In the case of two prey species we had two different matrices, $\mathbf{S}_{\mathrm{I}}^{*}$ and $\mathbf{S}_{\mathrm{II}}^{*}$, depending on whether the distance between the two prey species was equal to one or larger than one. For $n$ prey species there are $2^{n-1}$ different $\mathbf{S}^{*}$-matrices, because $n-1$ distances can assume a value equal to one or larger than one.

However, it is straight-forward to construct the $\mathbf{S}^{*}$-matrix for an omnivore feeding on $n$ arbitrary prey species, knowing how the $\mathbf{S}^{*}$-matrices for $n=2$ look like. The reason is, that all principal submatrices of second order of the $\mathbf{S}^{*}$ matrix for an $n$-prey omnivore correspond to $\mathbf{S}^{*}$-matrices of 2-prey omnivores. More precisely, deleting all rows and columns in the $n \times n$ matrix for an $n$-prey omnivore except for two, yields a $2 \times 2$ matrix which corresponds to either the one given in (4.14) or the one given in (4.15). 
As an example let us consider an omnivore feeding on trophic levels 1,2 and 4. The evolution equations for the patch densities $[1 x],[2 x]$ and $[4 x]$ give rise to the matrix

$$
\mathbf{S}^{*}=\left(\begin{array}{ccc}
{[1]^{*}-p_{2}^{*}-2 \bar{z}} & {[1]^{*}+\bar{z}} & {[1]^{*}+\bar{z}} \\
{[2]^{*}+p_{2}^{*}} & {[2]^{*}-p_{3}^{*}-3 \bar{z}} & {[2]^{*}+\bar{z}} \\
{[4]^{*}} & {[4]^{*}} & {[4]^{*}-p_{5}^{*}-5 \bar{z}}
\end{array}\right)
$$

at $x$-free equilibrium. The principal submatrices of second order $\mathbf{S}_{(k)}^{*}$ are obtained by deleting the $k$-th row and the $k$-th column:

$$
\begin{aligned}
& \mathbf{S}_{(1)}^{*}=\left(\begin{array}{cc}
{[2]^{*}-p_{3}^{*}-3 \bar{z}} & {[2]^{*}+\bar{z}} \\
{[4]^{*}} & {[4]^{*}-p_{5}^{*}-5 \bar{z}}
\end{array}\right), \\
& \mathbf{S}_{(2)}^{*}=\left(\begin{array}{cc}
{[1]^{*}-p_{2}^{*}-2 \bar{z}} & {[1]^{*}+\bar{z}} \\
{[4]^{*}} & {[4]^{*}-p_{5}^{*}-5 \bar{z}}
\end{array}\right), \\
& \mathbf{S}_{(3)}^{*}=\left(\begin{array}{cc}
{[1]^{*}-p_{2}^{*}-2 \bar{z}} & {[1]^{*}+\bar{z}} \\
{[2]^{*}+p_{2}^{*}} & {[2]^{*}-p_{3}^{*}-3 \bar{z}}
\end{array}\right) .
\end{aligned}
$$

It is evident from the general formulae in (4.14) and (4.15) that the above $2 \times 2$ matrices correspond to the transition matrices for 2-prey omnivores feeding on species 2 and 4, on species 1 and 4, and on species 1 and 2, respectively.

The calculation of coexistence ranges for omnivores feeding on $n$ species follows the same argumentation as in the case of omnivores feeding on two prey species. A splitting of the form (4.6) reveals that all eigenvalues of $\check{\mathbf{s}}_{\mathbf{1}}$ are negative. Therefore, $\mathbf{S}^{*}$ is stable at $z=0$. The first (i.e. the smallest) $z$-value where $\operatorname{det}\left(\check{\mathbf{S}}^{*}\right)$ changes sign implies that $\mathbf{S}^{*}$ becomes unstable and thus determines the lower bound of the coexistence range. Because $\operatorname{det}\left(-\check{\mathbf{s}}_{1}^{*}\right)>0$, it follows from (4.7) that $\bar{z} \check{\mathbf{M}}$ becomes larger than 1 when $\lambda\left(\mathbf{S}^{*}\right)$ crosses the $z$-axis. This leads to the following persistence threshold,

$$
z>\frac{\langle k\rangle}{\lambda(\check{\mathbf{M}})}
$$

which is the same as the one we obtained earlier for 2-prey omnivores.

When determining the upper bound of the persistence range, there is a little difference to the case of two prey species: A 2-prey omnivore will at the latest die out when its principal prey dies out. The ecological explanation for this is that a 2-prey omnivore becomes essentially a specialist when one of its prey species dies out, but competitive exclusion still penalizes it. In the $n$-omnivore case the situation is different (for $n>2$ ): When the principal prey dies out, the 
omnivore still has an advantage due to feeding on $n-1$ prey, and thus it can possibly persist in the system (as an $n-1$-omnivore) beyond $\bar{z}=2 /(i(i-1))$.

Mathematically, this difference is reflected in the fact that the $x$-free equilibrium can, in contrast to the case of a 2-prey omnivore, be unstable at the upper boundary of the validity range of $\hat{\mathbf{S}}^{*}$ : Because at $\bar{z}=2 /(i(i-1))$ species $i$ and species $i-1$ do not exist, the matrix $\hat{\mathbf{S}}^{*}$ becomes block-triangular, with one block being $n$-1-dimensional and one block being a single diagonal element. The diagonal element is negative $(-i \bar{z})$. The $n-1$-dimensional block corresponds to the principal submatrix $\mathbf{S}_{(n)}^{*}$, which is obtained by deleting The $n$-th row and the $n$-th column in the original $\mathbf{S}^{*}$-matrix ${ }^{11}$. Accordingly, the $(n-1) \times(n-1)$-block corresponds to an omnivore feeding on the $n-1$ remaining prey species, except for the principal prey $i-1$. So, the stability of $\hat{\mathbf{S}}^{*}$ at $\bar{z}=2 /(i(i-1))$ depends on the stability of $\mathbf{S}_{(n)}^{*}$ at that point. This leads to two possible scenarios:

Case 1) $\mathbf{S}_{(n)}^{*}$ is stable, i.e. the $n-1$-prey omnivore is not able to exist at $\bar{z}=$ $2 /(i(i-1))$. Then, the $n$-prey omnivore also does not exist at $\bar{z}=2 /(i(i-1))$. This means, all eigenvalues of $\hat{\mathbf{S}}^{*}$ are negative at $\bar{z}=2 /(i(i-1))$, and the first value where $\operatorname{det}\left(\hat{\mathbf{S}}^{*}\right)$ changes sign (for decreasing $z$ ) yields the invasion threshold for the $n$-prey omnivore. Because $\operatorname{det}\left(-\hat{\mathbf{s}}_{1}^{*}\right)<0$, it follows from $(4.7)$ that $\bar{z} \check{\mathbf{M}}$ becomes smaller than 1 when $\lambda\left(\mathbf{S}^{*}\right)$ crosses the $z$-axis. So, in this case we obtain the invasion threshold

$$
z<\frac{\langle k\rangle}{\lambda(\hat{\mathbf{M}})}
$$

which corresponds to the previous result for 2-prey omnivores.

Case 2) $\mathbf{S}_{(n)}^{*}$ is unstable, i.e. the $n$-1-prey omnivore is able to exist at $\bar{z}=2 /(i(i-1))$. Then, the $n$-prey does not die out in the interval $2 /(i(i+1)) \leq$ $\bar{z} \leq 2 /(i(i-1))$, even though the abundance of its principal prey decreases and vanishes at $\bar{z}=2 /(i(i-1))$. This implies that the $z$-value where $\operatorname{det}\left(\hat{\mathbf{S}}^{*}\right) \operatorname{changes}$ sign lies outside the validity range of $\hat{\mathbf{S}}^{*}$, more precisely $1 / \lambda(\hat{\mathbf{M}})>2 /(i(i-1))$. This means that the omnivore is able to persist as an $n-1$-prey omnivore in the neighboring interval until the $n-1$-prey omnivore eventually dies out. In this case, the upper bound of the coexistence range is given by the upper bound of the coexistence range of the corresponding $n-1$-prey omnivore. For the $n-1$-prey omnivore the situation is analogous to the $n$-prey omnivore: either it dies out before its principal prey species dies out (case 1), or it survives as $n-2$-prey omnivore (case 2).

So, the invasion threshold for an $n-1$-prey omnivore is obtained by iteratively considering the sequence of $n-k$-prey omnivores, where $1 \leq k \leq n-2$, until eventually case 1 is fulfilled. The iteration terminates at latest when $k=n-2$,

\footnotetext{
${ }^{11}$ Here, it is not necessary to distinguish between $\check{\mathbf{S}}^{*}$ and $\hat{\mathbf{S}}^{*}$, as the two matrices differ only in the $n$-th row. So, deleting the $n$-th row and the $n$-th column yields the same result for $\check{\mathbf{S}}^{*}$ and $\hat{\mathbf{S}}^{*}$.
} 
as for a 2-prey omnivore, the invasion threshold is always given by (4.22). Consequently, an $n$-prey omnivore can maximally survive until the prey species at second-lowest trophic level dies out. This is the case when the last omnivore considered in the sequence of $n-k$-prey omnivores is a 2-prey omnivore with prey species at subsequent trophic levels.

In our example of a 3-prey omnivore feeding on species 1, 2 and 4, the corresponding matrix at $\bar{z}=1 / 10$ is given by the block-triangular matrix

$$
\hat{\mathbf{S}}^{*}=\left(\begin{array}{ccc}
{[1]^{*}-p_{2}^{*}-2 \bar{z}} & {[1]^{*}+\bar{z}} & {[1]^{*}+\bar{z}} \\
{[2]^{*}+p_{2}^{*}} & {[2]^{*}-p_{3}^{*}-3 \bar{z}} & {[2]^{*}+\bar{z}} \\
0 & 0 & -5 \bar{z}
\end{array}\right) .
$$

The single diagonal element is negative and the $2 \times 2$-block corresponds to the principal submatrix $\mathbf{S}_{(3)}^{*}$, describing an omnivore which feeds on species 1 and species 2. This type of omnivore was considered in our first example, where we determined the persistence threshold as $\bar{z}=1 /(6+3 \sqrt{2})$. Therefore, $\mathbf{S}_{(3)}^{*}$ is unstable at $\bar{z}=1 / 10$ and consequently $\hat{\mathbf{S}}^{*}$ is unstable at that point (case 2 ). The invasion condition for the 3-prey omnivore in our example is thus given by the invasion condition for the 2-prey omnivore feeding on species 1 and 2, which yields $\bar{z}=1 / 3$ (case 1 ).

This example demonstrates the stabilizing effect of additional prey species. It is easy to verify with the formulae given in (4.17) and (4.18), that a 2-prey omnivore with prey at trophic levels 1 and 4 or a 2-prey omnivore with prey at trophic levels 2 and 4 goes extinct before its principal prey species dies out, while the 3-prey omnivore feeding on species 1, 2 and 4 does not.

In general, an $n$-prey omnivore with two principal prey species (i.e. the ones at highest trophic levels) at subsequent trophic levels does not die out before its principal prey species goes extinct. This stems from the fact that if an omnivore feeds on levels $i-1$ and $i-2$, the equilibrium patch density of the corresponding $n-1$-prey omnivore is maximal at $\bar{z}=2 /(i(i-1))$, and therefore prevents extinction. This situation is illustrated in Fig. 4.9. The converse is not true, i.e. an omnivore with two principal prey species at non-subsequent levels might also not die out before its principal prey goes extinct. The latter was for instance the case in our example of a 3-prey omnivore feeding on trophic levels 1,2 and 4 .

In this section, we showed that the coexistence range for $n$-prey omnivores crucially depends on the number of the prey species, and in particular on the distribution of those among the trophic levels. Certain configurations of prey species can have a significant stabilizing effect, leading to large coexistence ranges. In particular, prey species at subsequent trophic levels, and in general prey species with small trophic distances lead to enhanced persistence conditions. 


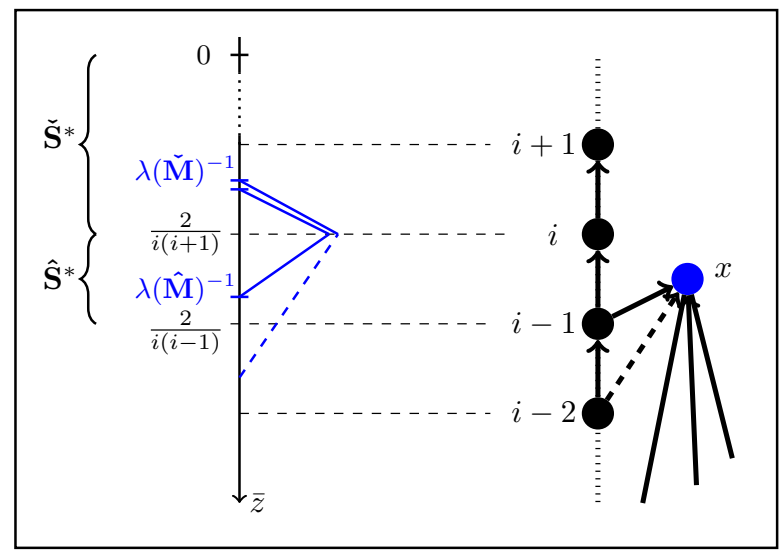

Figure 4.9: Coexistence threshold for omnivores feeding on $n$ prey species. Blue lines illustrate the equilibrium patch density for an omnivore with (dashed line) and without (solid line) prey species at level $i-2$. The lower bound of the persistence range is given by $\bar{z}=1 / \lambda(\check{\mathbf{M}})$. The upper bound of the persistence range is given by $\bar{z}=1 / \lambda(\hat{\mathbf{M}})$ if this value lies within the interval $2 /(i(i+1)) \leq \bar{z} \leq 2 /(i(i-1))$. Otherwise, the omnivore is able to survive as an $n-1$-prey omnivore beyond $\bar{z}=2 /(i(i-1))$, until it eventually dies out when the $n-1$-prey omnivore dies out. An additional prey at level $i-2$ results always in a value $\bar{z}=1 / \lambda(\hat{\mathbf{M}})$ outside the validity range of $\hat{\mathbf{S}}^{*}$.

Let us summarize at this point the main results we obtained in this chapter so far. We developed an approach for the estimation of persistence conditions in metacommunities that involves two principal ideas. The first step consists in determining the stability of the system in equilibrium before the arrival of a new species $s$. Linear stability analysis of the transition matrix $\mathbf{S}^{*}$ at $s$ free equilibrium leads to a persistence condition for species $s$ in terms of the parameter $z$. If this condition is an implicit condition for $z$ (which is in general the case), we demonstrated that it is convenient to consider the corresponding next generation matrix $\mathbf{M}$ at $s$-free equilibrium. So, the second step consists in calculating the spectrum of $\mathbf{M}$. The largest eigenvalue of $\mathbf{M}$ yields an explicit condition for the persistence range in terms of $z$. In contrast to the results reported in [157], the proposed approach facilitates a systematic analysis of persistence conditions with respect to the structure of the food web. 


\subsection{Influence of the patch-network on the persistence conditions}

In the first part of this chapter, we were mainly concerned with the influence of the food web, i.e. the interaction pattern between species, on persistence conditions in metacommunities. So far, we did not take into account the influence of the underlying patch-network on the persistence conditions, but we rather assumed the patch-network to be approximately homogeneous. In reality, the routes of dispersal across patches, as well as the resource-patches themselves can display heterogeneity $[159,160]$.

In this section, we study the formation of ecological metacommunities in heterogeneous patch-networks and discuss the implications of heterogeneity for the coexistence of several species in these metacommunities. First, we analyze the impact of different types of patches. Then, we consider patch-networks with heterogeneous degree distributions.

\subsubsection{Patch-networks with multiple types of habitat}

Let us consider a patch-network which is inhomogeneous in the sense that it provides different types of patches, corresponding to several types of habitat. So far, we considered all empty patches to be equal. Now, we allow for the patch-network to comprise $m$ different types of habitat, $h_{1}, h_{2}, \ldots h_{m}$.

We start our analysis by considering specialists. A maximal food web containing only specialists comprises $m$ independent food chains (see Fig. 4.10). In contrast to the case of a single habitat, the total number of species $s$ in general exceeds the maximal trophic level $l$. Starting from a patch-network with $m$ different types of habitat leads to a set of evolution equations of the form

$$
\begin{aligned}
\frac{d}{d t}\left[h_{f}\right] & =-c\langle k\rangle\left[h_{f}\right] p_{1}^{(f)}+e p_{1}^{(f)}, \\
\frac{d}{d t}[1]^{(f)} & =c\langle k\rangle\left[h_{f}\right] p_{1}^{(f)}-c\langle k\rangle[1]^{(f)} p_{2}^{(f)}+e p_{2}^{(f)}-e[1]^{(f)}, \\
& \vdots \\
\frac{d}{d t}[l-1]^{(f)} & =c\langle k\rangle[l-2]^{(f)} p_{l-1}^{(f)}-c\langle k\rangle[l-1]^{(f)} p_{l}^{(f)}+e p_{l}^{(f)}-(l-1) e[l-1]^{(f)}, \\
\frac{d}{d t}[l]^{(f)} & =c\langle k\rangle[l-1]^{(f)} p_{l}^{(f)}-l e[l]^{(f)},
\end{aligned}
$$

for each habitat type $h_{f}$. Symbols of the form $[i]^{(f)}$ refer to patch densities of habitat type $h_{f}$ where a chain up to trophic level $i$ is present. Accordingly $p_{i}^{(f)}$ denotes patch densities where a species at trophic level $i$ is present in a patch of type $h_{f}$, irrespective of other species at different trophic levels. So, for example, $[1]^{(1)}$ refers to habitat-patches of type $h_{1}$, which are inhabited by one basal species. Note that patches of a certain habitat type can only be invaded 


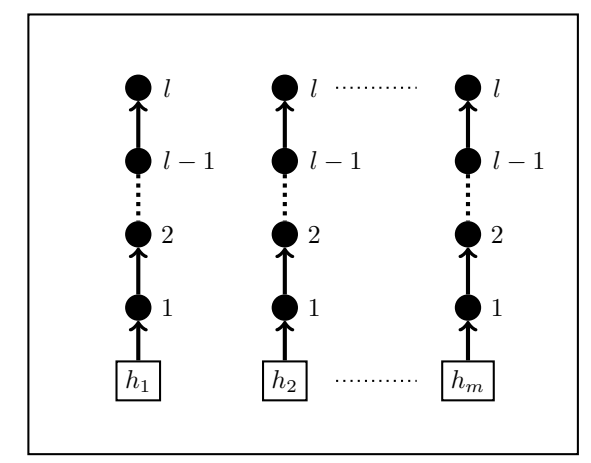

Figure 4.10: Food chains arising in a system with $m$ types of habitat. For equal distribution of habitat, all species of the same trophic level can be treated in the same way. Consequently, the length of all $m$ food chains is the same for given $z$, and the total number of species yields $s=l m$.

by a basal species of one specific type, i.e. by one of the $m$ different specialists at trophic level 1.

Solving (4.23) at equilibrium yields the following equilibrium patch densities for the $m$ types of empty habitat,

$$
\left[h_{f}\right]^{*}=\bar{z}, \quad 1 \leq f \leq m,
$$

and for the $l^{(f)}$ specialists feeding upon each other in a habitat patch $h_{f}$ :

$$
[i]^{(f)^{*}}=\left\{\begin{array}{lc}
(i+1) \bar{z}, & 1 \leq i<l^{(f)}, \\
{[f]-\frac{1}{2} i(i+1) \bar{z},} & i=l^{(f)} .
\end{array}\right.
$$

Here, $[f]$ denotes the initial fraction of habitat $h_{f}$. For simplicity, from now on we assume equal distribution of habitat patches, i.e. $[f]=1 / m$. As a consequence, species at the same trophic level, but from different chains have the same prerequisites and can be treated in the same way. Accordingly, the chain lengths are the same for all habitat types and the total number of species in the metacommunity is given by $s=l m$. Then, we can suppress the superscript $(f)$ and (4.24) becomes

$$
[i]^{*}=\left\{\begin{array}{lc}
(i+1) \bar{z}, & 1 \leq i<l, \\
\frac{1}{m}-\frac{1}{2} i(i+1) \bar{z}, & i=l .
\end{array}\right.
$$

Compared to the equilibrium patch densities in (4.2), the only difference is the " $1 / m$ ", which is due to normalization. Following the same lines as in the case 
of one habitat type, the invasion condition for a specialist $l$ yields

$$
z<\frac{2\langle k\rangle}{m l(l+1)}
$$

This means, for $z<2\langle k\rangle /(m l(l+1))$ specialists can invade a system of $l-1$ trophic levels and occupy the top of each of the $m$ independent food chains. Compared to the invasion threshold in (4.3) for a single habitat, the invasion threshold acquires a factor $1 / m$ due to the presence of $m$ independent specialist chains. One can interpret this result as an effectively decreased mean degree $\langle k\rangle / m$. This intuitively makes sense, as in average there are $m$ times less resource patches available for each species. We note here that persistence thresholds and invasion thresholds for omnivores are also reduced by a factor $1 / m$ when the system comprises $m$ types of habitat. One can see this by plugging the equilibrium densities given in (4.25) in the $\mathbf{S}^{*}$-matrices in (4.14) and (4.15). Then, $\mathbf{s}_{1}$ acquires a factor $1 / m$ which directly translates to modified upper and lower bounds by a factor $1 / m$.

Now, we introduce generalists, which are capable of feeding on more than one prey species. In contrast to the omnivores considered in the previous sections, the generalists we consider here are capable of feeding on prey species from several independent food chains. For now, we restrict our analysis to generalists feeding on two prey species from two independent chains in a system with two different types of habitat. We denote the trophic levels of the prey species of the generalist $g$ with $i-1$ and $j-1$, where $j \leq i$, and the two prey species are from different chains.

For the calculation of persistence ranges for generalists we employ the formalism developed above. The transition matrix $\mathbf{S}^{*}$, describing the evolution of $[(j-1) g]$ - and $[(i-1) g]$-patches in the $g$-free equilibrium is given by

$$
\mathbf{S}^{*}=\left(\begin{array}{cc}
{[j-1]^{*}-p_{j}^{*}-j \bar{z}} & {[j-1]^{*}} \\
{[i-1]^{*}} & {[i-1]^{*}-p_{i}^{*}-i \bar{z}}
\end{array}\right) .
$$

In contrast to the case of omnivores, there is no possibility for $[(j-1) g]$-patches to become $[(i-1) g]$-patches or vice versa, as both prey species are from independent chains. Otherwise, the situation is similar to the one for omnivores: generalists require a certain amount of suitable resource patches to establish themselves, while at the same time they face competition from the respective superior specialists. Thus, as before, we expect the persistence condition to be dependent on the presence or absence of specialist $i$ and accordingly to give rise to an upper and lower bound for the parameter $z$, defining a coexistence range.

For the calculation of the coexistence range for a generalist, we have to distinguish two cases (see Fig. 4.11): A) The two prey species are at the same 


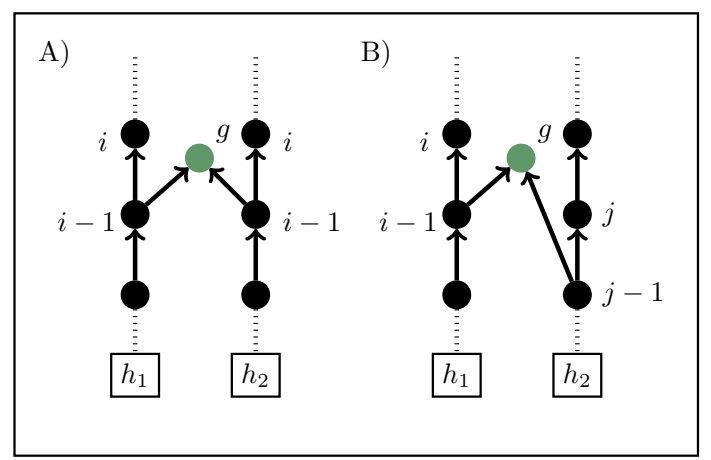

Figure 4.11: Food web configuration consisting of two independent chains and a generalist $g$. The generalist feeds on two prey species from different chains, arising from two types of habitat. A) The trophic level of both prey species is the same $(i=j)$. B) The trophic levels differ at least by one $(i>j)$. The corresponding formulae for the coexistence ranges for both configurations are derived in the text.

trophic level $(i=j)$. B) The two prey species are at different trophic levels $(i>j)$. This distinction is necessary, because a generalist feeding upon two prey species at the same level, say $i-1$, experiences no competition from a specialist competitor if species $i$ is not present. In contrast, a generalist feeding upon one prey at level $i-1$ and a second one at some lower level $j-1$, even in the absence of species $i$, experiences competition from the specialist competitor $j$ in the second chain.

An analogous argumentation as in the case of omnivores leads to the following expressions for the coexistence ranges:

A)

$$
\frac{\langle k\rangle}{i(i+3)}<z<\frac{\langle k\rangle}{i^{2}}
$$

B)

$$
\frac{\langle k\rangle}{\lambda(\check{\mathbf{M}})}<z<\frac{\langle k\rangle}{\lambda(\hat{\mathbf{M}})}
$$

Here, we used that the largest eigenvalues of the respective next generation matrices in case A) have a simple form. The corresponding matrices $\check{\mathbf{M}}$ and $\hat{\mathbf{M}}$ in case B) follow from (4.27), taking into account that for two types of habitat $(m=2)$ the equilibrium patch densities are given in (4.25):

$$
\check{\mathbf{M}}=\left(\begin{array}{cc}
j(j+1) & 2 j \\
2 i & i(i+1)
\end{array}\right), \quad \hat{\mathbf{M}}=\left(\begin{array}{cc}
j(j-1) & -2 j \\
2 i & i(i+1)
\end{array}\right) .
$$




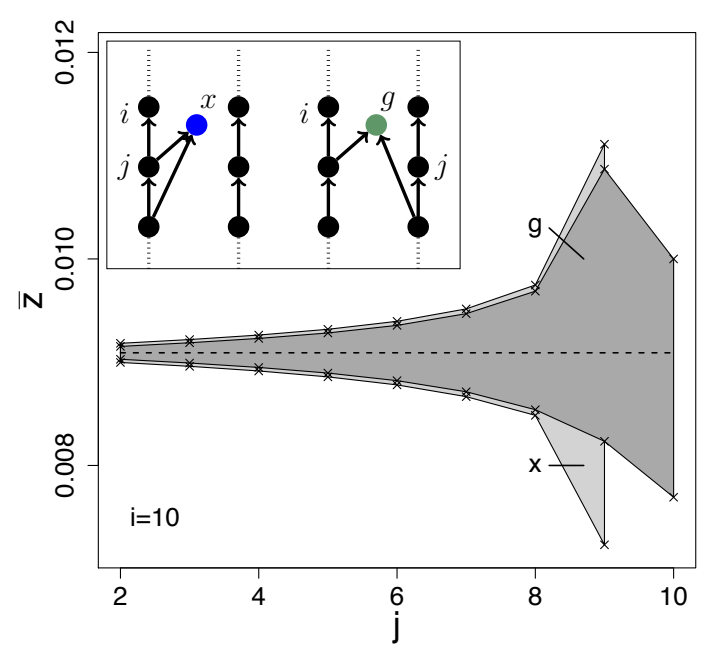

Figure 4.12: Comparison between the coexistence ranges for omnivores and generalists. For a system of two types of habitat and 10 trophic levels (20 specialists in two food chains), we plot the coexistence range for an omnivore $x$, feeding upon prey species from exclusively one food chain and the coexistence range for a generalist $g$, feeding upon two prey species from two food chains (inset). Throughout the whole $j$-range, the coexistence range for the omnivore is slightly larger than the coexistence range for the generalist.

Analyzing the dependence of the coexistence ranges for generalists with respect to the trophic levels of their prey species, we find qualitatively the same characteristics as for omnivores: The coexistence range increases with decreasing trophic levels and with decreasing distance between the levels. One exception is situation $\mathrm{A}$ ), where both prey levels are equal $(i=j)$. In that case, the coexistence range is smaller than for $i=j+1$, though larger than for $i>j+1$ (see Fig. 4.12). So, as in the case of omnivores, the maximal coexistence range for generalists is encountered when the two prey species are at subsequent trophic levels.

Fig. 4.12 shows a comparison between the coexistence ranges for generalists and the corresponding coexistence ranges for omnivores. We find that the coexistence range for omnivores is always (slightly) larger than the corresponding one for generalists. This stems from the fact that omnivores benefit from local extinction of species $j$ in $[(i-1) x]$-patches (which become $[(j-1) x]$-patches), leading to one larger entry in the matrix $\check{M}$ and one smaller entry in the matrix $\hat{\mathbf{M}}$. Therefore, both thresholds contribute in the case of the omnivore to a larger coexistence range compared to the generalist.

A generalization to generalists feeding on $n$ prey species in $n$ independent chains is straight-forward: as in the case of $n$-prey omnivores, the corresponding 
transition matrices for $n$-prey generalists can be constructed from the 2-prey transition matrix given in (4.27). Combining our results for omnivores with the results from this section, we can further calculate coexistence ranges for generalists which feed on prey species in different food chains and on several trophic levels in the same food chain. Then, every principal submatrix of second order of the transition matrix for such a generalist corresponds to either of the 2-prey transition matrices given in (4.14), (4.15) or (4.27).

In summary, the impact of different types of habitat can be described as follows: coexistence ranges (for specialists, omnivores and generalists) decrease by a factor $1 / m$ when the system comprises $m$ types of habitat. For a specific species, introducing $m$ types of habitat has the same effect as decreasing the mean degree by a factor $1 / \mathrm{m}$. However, several types of habitat convey species diversity in the sense that food webs with multiple types of habitat facilitate a large number of species which potentially coexist in the metacommunity.

\subsubsection{Patch-networks with heterogeneous degree distributions}

In this section we relax our previous assumptions on degree-homogeneity and account for the full degree distribution of the patch-network. The aim is to derive analogous expressions for coexistence thresholds as in the degree-homogeneous case and to analyze the size of coexistence ranges with respect to changes in the degree distribution. Deriving the corresponding expressions for invasion thresholds reveals that an exact treatment of degree-heterogeneous networks is in general difficult, and only in special cases feasible. Nevertheless, we are able to extract information about the influence of the degree distribution on the formation of food webs by applying a useful approximation.

Let us first consider a linear chain of $l$ specialist species in a patch-network of one single type of habitat. We denote the probability that species $i$ inhabits patch $m$ by $p_{i}^{m}$. Then, the time evolution for the probability vector $\overrightarrow{p_{i}}$ is given by the following equation:

$$
\dot{\vec{p}}_{i}=\left(\left(\mathbf{p}_{i-1}-\mathbf{p}_{i}\right) \mathbf{A}-i z \mathbf{1}\right) \vec{p}_{i}, \quad 0<i \leq l .
$$

Here, $\mathbf{A}$ is the adjacency matrix of the patch-network, which captures the complete structure of the network, including degree correlations between patches. The matrices $\mathbf{p}_{i}$ denote diagonal matrices with the corresponding entries of the vector $\overrightarrow{p_{i}}$ on the diagonal and $\mathbf{p}_{0}=\mathbf{1}$, where $\mathbf{1}$ is the identity matrix.

Given an arbitrary network topology, the equilibrium probabilities for a specific patch $m$ are fully determined by the self-consistent equation,

$$
p_{i}^{m *}=\frac{p_{i-1}^{m *} \sum_{n} a^{m n} p_{i}^{n *}}{i z+\sum_{n} a^{m n} p_{i}^{n *}} .
$$


One can interpret the quantity $\sum_{m} a^{m n} p_{i}^{n *}$ as a state-sensitive degree. In contrast to the total degree $k^{m}$ of a node $m$, which captures the number of neighboring patches independent of their states, the state-sensitive degree $k_{i}^{m}$ captures only the number of neighboring patches where species $i$ is established.

Accordingly, we define the state-sensitive degree as

$$
k_{i}^{m}:=\sum_{n} a^{m n} p_{i}^{n *} .
$$

Note that the state-sensitive degrees are in general not integers, as they involve the equilibrium probabilities $p_{i}^{n *}$. So, they rather correspond to expected degrees at equilibrium. In analogy to the adjacency matrix which captures all the connections between the patches, we can further introduce a state-sensitive adjacency matrix, which captures the (expected) connections to patches where certain species are established. The rowsum of each row of the state-sensitive adjacency matrix then yields the respective state-sensitive degree:

$$
k_{i}^{m}=\sum_{n} a_{i}^{m n}, \quad \text { where } \quad\left(a_{i}^{m n}\right)=\mathbf{A}_{i}=\mathbf{A p}_{i}^{*}
$$

The resulting overlay networks defined by the state-sensitive adjacency matrices $\mathbf{A}_{i}$ are weighted and directed.

After these introductory remarks and definitions, let us come back to the original question, the persistence conditions of species depending on the patchnetwork topology. According to (4.29) the invasion condition for species 1 yields,

$$
\lambda(\mathbf{A}-z \mathbf{1})>0,
$$

from which we directly obtain the condition

$$
z<\lambda(\mathbf{A}) \text {. }
$$

This result was first obtained in [130] in the context of epidemic spreading on heterogeneous networks. The threshold in (4.3) constitutes a first approximation to (4.31). This becomes clear when we consider that $\lambda(\mathbf{A})$ is bounded by the minimum and the maximum of the rowsums $R_{i}$ of $\mathbf{A}$ (Frobenius inequality [133]),

$$
\min _{i}\left(R_{i}(\mathbf{A})\right) \leq \lambda(\mathbf{A}) \leq \max _{i}\left(R_{i}(\mathbf{A})\right) .
$$

Approximating $\lambda(\mathbf{A})$ by $\langle k\rangle$ corresponds to a simple averaging over all rowsums. For narrow degree distributions, this approximation improves, as the distance between upper and lower bound decreases. For homogeneous distributions the approximation becomes exact.

Along the same lines, one could propose an average only over rows with equal rowsum, i.e. joining rows (and columns) with equal rowsums to a single 
row (column). This leads to a, in general not symmetric, reduced matrix $\mathbf{A}^{\prime}$, whose largest eigenvalue can be viewed as a second approximation to $\lambda(\mathbf{A})$, the largest eigenvalue of the original matrix. Indeed, it turns out that the reduced matrix is equivalent to the so-called connectivity matrix $\mathbf{C}_{k k^{\prime}}$, which was found to determine the epidemic threshold in degree-correlated networks $[79,80]$. However, we should emphasize, that the largest eigenvalue of the reduced matrix, or connectivity matrix, is only an approximation, as it captures the average degree correlations between neighboring nodes. The approximation becomes exact when the connectivity matrix is similar to the adjacency matrix $\left(\mathbf{C}_{k k^{\prime}} \sim \mathbf{A}\right)$. This is the case when each degree class corresponds to an orbit of the patch-network, i.e. all nodes with equal degree have exactly the same topological position in the patch-network.

From (4.31) we can also recover the epidemic threshold obtained in [48], applying the following approximation for the largest eigenvalue of a matrix $\mathbf{M}$ (see e.g. [160]):

$$
\lambda(\mathbf{M}) \approx \frac{\sum_{i} C_{i}(\mathbf{M}) R_{i}(\mathbf{M})}{\sum_{i} R_{i}(\mathbf{M})} .
$$

Here, $C_{i}(\mathbf{M})$ denote the columnsums of $\mathbf{M}$. This approximation becomes exact for uncorrelated networks.

So, for the invasion condition of the first species we derived an expression which corresponds to a result found in the literature on epidemic thresholds [130]. We showed that this expression encompasses all relevant approximations to the epidemic threshold, which are widely employed [48, 79, 80, 161, 162].

Now, we consider the condition for a second species to establish in a system, where one species resides in equilibrium. With (4.29) the invasion condition for species 2 becomes

$$
z<\frac{\lambda\left(\mathbf{p}_{1} \mathbf{A}\right)}{2}=\frac{\lambda\left(\mathbf{A}_{1}\right)}{2} .
$$

Instead of the adjacency matrix, now the state-sensitive adjacency matrix $\mathbf{A}_{1}$ determines the threshold condition. This means that the invasion condition for species 2 depends on the degree distribution and on the degree correlations with respect to patches where species 1 is established. Analogously, the respective invasion condition for a species at trophic level $i$ yields

$$
z<\frac{\lambda\left(\mathbf{A}_{i-1}\right)}{i}
$$

where $\mathbf{A}_{i-1}$ is the adjacency matrix with respect to patches inhabited by species $i-1$. Note that (4.32) comprises an implicit condition for $z$, as $\mathbf{A}_{i-1}$ is $z$ dependent. So, although the formula in (4.30) in principle defines $\vec{p}_{i}{ }^{*}$ for any given adjacency matrix, and therefore $\mathbf{A}_{i-1}$ can be computed, in practice it is often not possible to obtain the exact values for the invasion thresholds of 
specialists, especially for large networks and for high trophic levels. Consequently, the coexistence ranges for omnivores or the persistence conditions for more complex food webs are even harder to compute exactly.

In order to describe real ecological systems it often suffices to know statistical properties about the underlying patch-network, because the exact topology of the dispersal routes is in most cases not empirically accessible. We stated above that a first approximation to the largest eigenvalue of a matrix is the average over the rowsums. In our case, this would correspond to the mean state-sensitive degree. However, we also saw before that this approximation fails for heterogeneous degree distributions. In general, one could argue, that the largest eigenvalue of the corresponding adjacency matrix is the quantity which more appropriately captures a broad degree distribution than the simple mean degree, at least with respect to spreading characteristics.

If we assume broad degree distributions to be characterized by the largest eigenvalue of their corresponding adjacency matrix, the following relationship,

$$
\lambda\left(\mathbf{A}_{i}\right) \approx \lambda\left(\mathbf{A}_{i-1}\right)-i z,
$$

provides a reasonable approximation in many cases. One way to obtain this relationship is by rearranging (4.30) into

$$
k_{i}^{m}+i z=p_{i-1}^{m} \frac{k_{i}^{m}}{p_{i}^{m}}=k_{i-1}^{m},
$$

where the last equality follows from the definition of the state-sensitive degree. Then, motivated by our previous reasoning, instead of averaging in the above equation we take the largest eigenvalues of the corresponding matrices, which directly yields (4.33).

With (4.33) we obtain the following (approximate) invasion condition for species $i$ :

$$
z<\frac{2 \lambda(\mathbf{A})}{i(i+1)}
$$

This is a result which goes beyond the studies of epidemic thresholds, as it shows that not only the invasion of the first species, but also the invasion of all subsequent species is to large extent influenced by the largest eigenvalue of the adjacency matrix. Using assumption (4.33), one can directly transfer the results given in (4.17) and (4.28) for the coexistence ranges of generalists to heterogeneous networks, just by replacing $\langle k\rangle \rightarrow \lambda(\mathbf{A})$.

The invasion threshold in (4.34) is larger compared to the one given in (4.3) for a homogeneous patch-network of the same mean degree. Analogously, the coexistence ranges for generalists are larger in heterogeneous networks. This leads to the conclusion, that heterogeneous networks tend to convey the invasion and persistence of species, and thus foster species diversity. This result is 
in agreement with observations made in heterogeneous metapopulation models. In [159] was reported that "clumping of patches increases persistence" in metapopulations.

For metapopulation models, invasion conditions in terms of the largest eigenvalue of a landscape matrix, the so-called invasion capacity were proposed in $[160,163]$. While these studies are more general in the sense that patches of different sizes and different distances among each other were considered, they lack trophic interactions within patches. One can relate the state-sensitive adjacency matrices here, which yield the invasion conditions in the metacommunity model, to landscape matrices in a metapopulation model, where patch sizes and distances arise as a result of the trophic interactions. Then, results from metapopulation theory, as for example the impact of habitat loss [160], can be translated to metacommunity models.

\subsection{Discussion}

In this chapter, we investigated the emergence and persistence of species diversity in ecological systems, using a metacommunity model which is based on the one proposed in [157]. Our model involves two types of networks: a network of interactions between species (the food web) and a network of dispersal routes between resource patches (the patch-network). Our investigations demonstrate that the structural properties of both types of networks influence the parameter range where several species are able to coexist, and hence affect species diversity.

In order to quantify the sensitivity of species diversity, we developed a mathematical approach for the calculation of persistence ranges. The calculation is based on a successive linear stability analysis of the system in equilibrium, before arrival of a new species. Introducing the notion of a next generation matrix yields explicit expressions for persistence conditions in terms of the parameters of the model. The proposed approach provides a simple means to analyze persistence conditions in several respects.

First, we focused on the dependence of persistence ranges on the food web topology. Our analysis revealed, for example, that relatively large persistence ranges can be obtained for all types of predators as long as the predators feed on "low-level" prey and on "similar-level" prey. In particular, predators feeding on many prey species at subsequent trophic levels are able to persist in a wide range of parameter space.

Second, we studied the impact of the patch-network on the persistence ranges. In degree-homogeneous networks, an increased mean degree directly results in an increased persistence range for any species type. A reverse effect is observed when the patch-network comprises many different types of habitat. Then, the mean degree is effectively reduced, which results in a smaller persistence range. Reasonable approximations reveal that in degree-heterogeneous networks the 
largest eigenvalue of the corresponding adjacency matrix replaces the mean degree in the expressions for the persistence ranges, suggesting that degreeheterogeneous networks tend to display larger persistence ranges.

The approach we described in this chapter in principle allows for the estimation of the persistence condition for an arbitrary given food web configuration. However, the food web configurations we discussed here are still missing two aspects, which might be present in real-world food webs: a) Predators which feed on generalists (here we considered exclusively specialist prey). b) Competition between generalists (here we considered only competition between specialists and generalists). Let us briefly address these two issues and point out possibilities for the extension of our formalism to these more general food webs.

Concerning a), in order to determine the stability of the $s$-free equilibrium in dependence on $z$, where $s$ is now a predator possibly feeding on generalists, one has to analyze an $\mathbf{S}^{*}$-matrix which in general contains not only equilibrium patch densities of specialists, but also equilibrium patch densities of generalists. As we derived a simple expression for equilibrium patch densities of omnivores (more generally, the expression applies analogously to generalists), the corresponding $\mathbf{S}^{*}$-matrix can be constructed. Note that the obtained $\mathbf{S}^{*}$-matrix is in general not linear in $z$ when we allow for generalist prey species, because the equilibrium patch density of a generalist is not linear in $z$. Then, one can either solve the condition for $z$ numerically from the condition $\lambda\left(\mathbf{S}^{*}\right)>0$ or employ the approach we introduced here, by calculating the largest eigenvalue of the corresponding iteration matrix $\mathbf{M}$. For the latter, $\mathbf{S}^{*}$ has to be linearized, which is expected to give a good approximation of the persistence threshold, as we saw that the equilibrium patch density of a generalist is almost linear in $z$.

Regarding b), the competition rules introduced in [157] can be extended to account for competition between all types of predators feeding on the same prey. Following the same argumentation as for the competition between a specialist and a generalist, it is reasonable to assume that the more specialized predator is the superior competitor with respect to the common prey. In order to determine which of two competing predators $x$ and $y$ is more specialized, one can compare the respective fractions of patches involving the common prey and competitor $x$ and the fraction of patches involving the common prey and competitor $y$. Then, we call a predator $x$, feeding on prey $i-1$ and $j-1$, more specialized on prey $i-1$ than a predator $y$, feeding on $i-1$ and $k-1$, if the fraction of $[(i-1) x]$-patches with respect to $p_{x}$ at the $y$-free equilibrium is larger than the fraction of $[(i-1) y]$-patches with respect to $p_{y}$ at the $x$-free equilibrium. More precisely, omnivore $x$ is more specialized on the prey species at level $i-1$ than omnivore $y$, if

$$
\frac{[(i-1) x]^{*}}{p_{x}^{*}}>\frac{[(i-1) y]^{*}}{p_{y}^{*}}
$$


where $p_{x}^{*}=[(i-1) x]^{*}+[(j-1) x]^{*}$ and $p_{y}^{*}=[(i-1) y]^{*}+[(k-1) y]^{*}$. This is, because the total $x$-population is to larger extent sustained by prey $i-1$ than the $y$-population, and therefore $x$ is assumed to stronger depend on the $i-1$-supply, which means that it is more specialized on $i-1$. All quantities in (4.35) can be calculated, so that one can determine the superior competitor in any given competition. Then, employing the described method, coexistence ranges for competing generalists are computable.

Within the scope of the discussed metacommunity model, one can understand the emergence of species diversity. Here, we identified criteria which promote or inhibit the coexistence of many different species in the metacommunity. While the model is not capable of providing a comprehensive description of real ecological systems, it is, because of its simplicity, a useful model to gain an understanding of principal mechanisms. At the same time, the model can serve as a reference point for the development of more realistic future models.

For example, an immediate adjustment to river networks comprises the incorporation of directed links. The expressions for the persistence ranges can be easily transferred to directed patch-networks. The role of the degree distribution is then assumed by the in-degree distribution, as only the number of incoming links matters, which facilitate the arrival of species.

A direction of further research constitutes the development of adaptive metacommunity models, where, for example, species are capable of adjusting their diet preferences according to the locally available resources. Studying adaptive metacommunity models might reveal essential ingredients for the evolution of specific, realistic, food webs. Presumably, the described framework for the calculation of persistence ranges in the original model can be applied in the same, or a slightly modified way to adaptive metacommunity models.

Finally, the mathematical framework presented here represents not only a useful tool for the analysis of future ecological models, but can be beneficial for studying dynamical processes in different kinds of systems. As we already pointed out, models for epidemic spreading are closely related to the metacommunity model discussed here. For example, in ongoing investigations on simultaneous spreading of several (competing) diseases [164-167] our results can be viewed from a different perspective: While in ecology the maintenance of species diversity is desirable, in epidemiology one aims to contain the diversity of diseases. Therefore, the proposed approach can be used to determine those parameter ranges where certain diseases go extinct, and to develop means for driving the system into the desired regime. 


\section{Conclusion}

In the present thesis, we developed a mathematical framework for the calculation of absorbing transitions in complex networks. Absorbing transitions are closely related to a loss of diversity in complex systems, as they imply an irretrievable extinction of certain components of the system. In order to predict these abrupt qualitative changes, it is essential to gain insight into basic mechanisms by studying simple model systems. Within the scope of two different network models, we demonstrated that transitions between phases of high diversity and phases of homogeneity can be analytically calculated, using concepts from percolation theory.

In Chapter 3, we developed an approach for the analytical calculation of fragmentation transitions in adaptive network models for opinion formation. A fragmentation transition characterizes the transition from a regime where many opinions survive in disconnected components of the network, from a regime where one single opinion survives. In order to estimate the transition point, we proposed to study the percolation of active links, starting from a network configuration close to the fragmentation transition. We found that it is crucial to choose an appropriate basis for the evolution equations of active links in order to predict the fragmentation transition to high accuracy. Previous approaches used a conventional moment expansion, where the basis consists of the network moments. This approach neglects correlations between active links, which increase close to the transition point. The basis set we proposed specifically accounts for these correlations and thus provides a much more accurate estimate of the fragmentation transition.

Using the percolation approach to study fragmentation transitions, first in the original adaptive voter model and then in variants of this model, we demonstrated the wide applicability of the method and identified possible limitations. In voter models with an arbitrary number of states our method revealed a class of equivalent models with respect to fragmentations. The difficulty to make exact predictions about fragmentation transitions in multi-state voter models originates in the unknown active link densities. Considering a directed voter model, we were able to test our approach for broad degree distributions. As expected, we found that the cut-off determines to large extent the precision of the fragmentation point, which implies limitations for the analytical investigation of fragmentation transitions in large heterogeneous networks. Finally, we showed that by means of the percolation approach, active link densities can be calculated. Although these results are only reliable close to the fragmentation point, an appropriate combination of both, percolation approach and pairapproximation can provide a reasonable expression for the active link density in the whole parameter range. 
The central result of Chapter 3 is the alternative analytical approach for the calculation of fragmentation transitions, which yields much more precise results than the common pair-approximation. The presented percolation method constitutes a basic framework for the treatment of fragmentation transitions, leaving room for a wide range of modifications in order to study a broad class of different models. We discussed necessary ingredients and possible difficulties for such modifications. For example, if the model displays degree-degree correlations, degree-state correlations or clustering, one has to choose a basis which accounts for these peculiarities.

In Chapter 4, we introduced a mathematical formalism for the calculation of persistence conditions for several ecological species. We employed a metacommunity model where complex food webs arise as a result of species dispersal across a network of habitat patches. Persistence conditions for species naturally depend on the predator-prey interactions and on the spatial patch-network. The proposed framework allows for a direct calculation of the parameter range where a food web of given structure is able to persist.

The basic idea of the approach is again inspired by percolation: We iteratively determine the stability of the equilibrium state with respect to arrival (and spreading) of an additional species. Then, introducing the concept of a next generation matrix yields explicit expressions for persistence conditions in metacommunities. The obtained general expressions offer a means for analyzing the dependence of the persistence range on both, the structure of the food web and the structure of the patch-network.

Analyzing the dependence of the persistence range on the structure of the food web revealed, for example, that generalists feeding on low trophic levels and on similar trophic levels exhibit larger persistence ranges compared to generalists feeding on high trophic levels and greatly differing trophic levels. Concerning the patch-network topology, we found that a high mean degree and degreeheterogeneity enhance the persistence conditions for all types of species.

Summarizing the results from Chapter 4, we applied a method previously used in epidemic modeling to determine persistence thresholds for ecological species. Our main achievement consists in the derivation of a simple formalism which in principle allows for the computation of persistence ranges for arbitrary food web configurations in ecological metacommunities. Identifying enhancing and inhibiting factors for species coexistence in simple models can contribute to develop strategies for maintenance of species diversity in real-world ecological systems.

The two different models, one describing opinion formation and the other one describing food web formation, can be regarded as two representatives of the same class of models, characterized by the presence of an absorbing transition. 
An absorbing transition is associated with a reduction of the state space. Once an absorbing state, or more generally, a set of absorbing states is reached, there is at least one state in the state space which cannot be reached any more. In network models, all potential (absorbing) final states are related to network motifs. As long as all possible motifs are present in the network, all final states (which are in agreement with the dynamical rules) are available. However, when specific types of motifs vanish, it can become impossible to reach certain final states. This relationship enables the calculation of absorbing transitions by studying the evolution of network motifs, and thus treating absorbing transitions as a percolation problem.

In the adaptive voter model, the absorbing transition is triggered by the extinction of certain types of links. When active links (possibly of several types, in the $G$-state model) vanish, the state space collapses to a (possibly partially) fragmented state. Therefore, studying percolation of active links close to their extinction allows for the calculation of the fragmentation threshold. In the metacommunity model, the absorbing transition is triggered by the extinction of certain types of nodes. When patches inhabited by one particular species vanish, all possible food webs involving this species are no longer possible to emerge in the metacommunity. Therefore, studying the evolution of patches with a particular species close to their extinction allows for the calculation of persistence ranges for food webs.

While extinction of an arbitrary network motif does not necessarily lead to an absorbing transition, an absorbing transition is always accompanied by the disappearance of one network motif or a set of network motifs. Therefore, it is generally possible to calculate absorbing transitions using a percolation approach for the corresponding set of motifs. In practice, one has to find the corresponding set of motifs and formulate the evolution equation for this set of motifs according to the dynamical rules, assuming a network configuration close to the transition. As demonstrated here, in the example of an adaptive voter model, the motif set is not unique and the precision of the obtained results crucially depends on the choice of the motif set. For fragmentation transitions, we found that an appropriate motif basis is given by fan motifs or spider motifs.

The correspondence between absorbing transitions and percolation is the central result of this thesis, offering a wide range of applications for the presented approach. The class of absorbing transitions includes phase transitions characterized by extinction, fragmentation or the onset of collective motion. Extinction concerns species in ecological models, cultural traits or languages in sociological models, or diseases in epidemiological models. Fragmentations can refer to a break up of social groups, but also to molecular interactions at interfaces of composite materials, like adhesion, in technological applications. Collective motion includes synchronization [168] or swarming processes [63, 64], where in 
a network description those motifs vanish, in which neighboring nodes are out of phase or not aligned, respectively.

Often, absorbing transitions mark drastic changes in the system under consideration and are therefore of particular relevance for applications in many different fields. These changes can be severe, as in the case of irretrievable extinction of species or languages, or they can be beneficial, as in the case of eradication of diseases. Accordingly, the aim is either to prevent an imminent transition or to mediate a transition. For both situations, an understanding of the underlying principles is crucial, in order to be able to control absorbing transitions. While simple toy-models already provide insight into basic mechanisms and dependencies, for the extraction of strategies for real-world applications, like vaccination strategies or ecosystem conservation, the development of more realistic models is necessary. In the process of continual model improvement, which typically takes decades, it is essential to have a mathematical toolbox at hand which "grows" with the model, meaning that the available methods adapt to the changing requirements. We think that the approach we presented here is suitable for the basic toolbox for network analysis, as it involves relatively few assumptions while being highly flexible at the same time.

Finally, this work demonstrates a virtue of network theory: One is able to study a great variety of different systems employing basically one and the same method. Or, to put it the other way round, developing new methods permits future investigations in many different fields. 


\section{References}

[1] A. Kohut and R. Wike. Assessing globalization: Benefits and drawbacks of trade and integration. Harvard Int. Rev., 30(1):70, 2008.

[2] D. Nettle and S. Romaine. Vanishing voices: The extinction of the world's languages. Oxford University Press, 2000.

[3] G. Ceballos and P. R. Ehrlich. Mammal population losses and the extinction crisis. Science, 296(5569):904-907, 2002.

[4] J. T. Bonner. The origins of multicellularity. Integr. Biol., 1(1):27-36, 1998.

[5] J. R. Sklaroff. Redundancy management technique for space shuttle computers. IBM J. Res. Dev., 20(1):20-28, 1976.

[6] A. Avizienis and J. C. Laprie. Dependable computing: From concepts to design diversity. Proc. IEEE, 74(5):629-638, 1986.

[7] M. Gell-Mann. What is complexity. Complexity, 1(1):16-19, 1995.

[8] C. H. Bennett. How to define complexity in physics, and why. Complexity, Entropy, and the Physics of Information, 8:137-148, 1990.

[9] E. Korutcheva. Advances in Condensed Matter and Statistical Physics. Nova Science Publishers Inc., 2004.

[10] L. A. N. Amaral and J. M. Ottino. Complex networks. Eur. Phys. J. B, 38(2):147-162, 2004.

[11] Y. Bar-Yam. Dynamics of complex systems. Addison-Wesley, 1997.

[12] A. Pikovsky, M. Rosenblum, and J. Kurths. Synchronization: A universal concept in nonlinear sciences. Cambridge University Press, 2003.

[13] S. C. Manrubia, A. S. Mikhailov, and D. H. Zannette. Emergence of dynamical order: synchronization phenomena in complex systems. World Scientific Publishing Co., 2004.

[14] A. Okubo. Dynamical aspects of animal grouping: swarms, schools, flocks, and herds. Adv. Biophys., 22:1-94, 1986.

[15] A. J. Koch and H. Meinhardt. Biological pattern formation: from basic mechanisms to complex structures. Rev. Mod. Phys., 66(4):1481, 1994. 
[16] E. Ravasz, A. L. Somera, D. A. Mongru, Z. N. Oltvai, and A. L. Barabási. Hierarchical organization of modularity in metabolic networks. Science, 297(5586):1551-1555, 2002.

[17] R. Guimera, L. Danon, A. Diaz-Guilera, F. Giralt, and A. Arenas. Selfsimilar community structure in a network of human interactions. Phys. Rev. E, 68(6):065103, 2003.

[18] A. Barrat, M. Barthlemy, and A. Vespignani. Dynamical processes on complex networks. Cambridge University Press, 2008.

[19] B. Drossel and A. J. McKane. Modelling food webs. Handbook of graphs and networks: From the genome to the internet, 2003.

[20] M. E. J. Newman. The structure and function of complex networks. SIAM Rev., pages 167-256, 2003.

[21] R. Albert and A. L. Barabási. Statistical mechanics of complex networks. Rev. Mod. Phys., 74:47-97, 2002.

[22] S. Bornholdt, H. G. Schuster, and J. Wiley. Handbook of graphs and networks. Wiley-VCH Verlag, 2003.

[23] T. Gross and H. Sayama. Adaptive Networks. Springer Verlag, 2009.

[24] M. E. J. Newman, A. L. Barabási, and D. J. Watts. The structure and dynamics of networks. Princeton University Press, 2006.

[25] D. K. Arrowsmith and C. M. Place. An introduction to dynamical systems. Cambridge University Press, 1990.

[26] S. H. Strogatz. Nonlinear dynamics and chaos: With applications to physics, biology, chemistry, and engineering. Westview Press, 1994.

[27] S. Boccaletti, V. Latora, Y. Moreno, M. Chavez, and D. U. Hwang. Complex networks: Structure and dynamics. Phys. Rep., 424(4-5):175-308, 2006 .

[28] M. E. J. Newman. Assortative mixing in networks. Phys. Rev. Lett., 89:208701, 2002.

[29] C. Nardini, B. Kozma, and A. Barrat. Who's talking first? consensus or lack thereof in coevolving opinion formation models. Phys. Rev. Lett., 100(15):158701, 2008.

[30] D. J. Watts. Networks, dynamics, and the small-world phenomenon 1. Am. J. Soc., 105(2):493-527, 1999. 
[31] S. Milgram. The small world problem. Psychol. Today, 2(1):60-67, 1967.

[32] P. Erdős and A. Rényi. On the evolution of random graphs. Publ. Math. Inst. Hungar. Acad. Sci., 1960.

[33] D. J. Watts and S. H. Strogatz. Collective dynamics of small-world networks. Nature, 393(6684):440-442, 1998.

[34] B. Bollobás. Random graphs. Cambridge University Press, 2001.

[35] A. L. Barabási and R. Albert. Emergence of scaling in random networks. Science, 286(5439):509-512, 1999.

[36] P. L. Krapivsky and S. Redner. Network growth by copying. Phys. Rev. E, 71:036118, 2005.

[37] S. Fortunato, A. Flammini, and F. Menczer. Scale-free network growth by ranking. Phys. Rev. Lett., 96:218701, 2006.

[38] A. L. Barabási, H. Jeong, Z. Néda, E. Ravasz, A. Schubert, and T. Vicsek. Evolution of the social network of scientific collaborations. Physica A, 311(3):590-614, 2002.

[39] E. Eisenberg and E. Y. Levanon. Preferential attachment in the protein network evolution. Phys. Rev. Lett., 91(13):138701, 2003.

[40] H. Jeong, Z. Néda, and A. L. Barabási. Measuring preferential attachment in evolving networks. Eur. Phys. Lett., 61:567, 2003.

[41] R. M. Anderson and R. M. May. Infectious diseases of humans: dynamics and control. Wiley-VCH Verlag, 1992.

[42] L. Sattenspiel and C. P. Simon. The spread and persistence of infectious diseases in structured populations. Math. Biosci., 90(1):341-366, 1988.

[43] I. M. Longini. A mathematical model for predicting the geographic spread of new infectious agents. Math. Biosci., 90(1):367-383, 1988.

[44] M. Kretzschmar and M. Morris. Measures of concurrency in networks and the spread of infectious disease. Math. Biosci., 133(2):165-195, 1996.

[45] L. Hufnagel, D. Brockmann, and T. Geisel. Forecast and control of epidemics in a globalized world. P. Natl. Acad. Sci., 101(42):15124-15129, 2004 . 
[46] S. Eubank, H. Guclu, V. S. A. Kumar, M. V. Marathe, A. Srinivasan, Z. Toroczkai, and N. Wang. Modelling disease outbreaks in realistic urban social networks. Nature, 429(6988):180-184, 2004.

[47] M. E. J. Newman, S. Forrest, and J. Balthrop. Email networks and the spread of computer viruses. Phys. Rev. E, 66(3):035101, 2002.

[48] R. Pastor-Satorras and A. Vespignani. Epidemic spreading in scale-free networks. Phys. Rev. Lett., 86(14):3200-3203, 2001.

[49] R. Pastor-Satorras and A. Vespignani. Epidemic dynamics and endemic states in complex networks. Phys. Rev. E, 63(6):066117, 2001.

[50] M. J. Keeling. Correlation equations for endemic diseases: externally imposed and internally generated heterogeneity. Proc. Roy. Soc. Lond. B, 266(1422):953-960, 1999.

[51] M. Kuperman and G. Abramson. Small world effect in an epidemiological model. Phys. Rev. Lett., 86:2909, 2001.

[52] M. E. J. Newman. Spread of epidemic disease on networks. Phys. Rev. E, 66:016128, 2002.

[53] V. Colizza, A. Barrat, M. Barthélemy, and A. Vespignani. The role of the airline transportation network in the prediction and predictability of global epidemics. P. Natl. Acad. Sci., 103(7):2015-2020, 2006.

[54] L. Bettencourt, A. Cintrón-Arias, D. I. Kaiser, and C. Castillo-Chávez. The power of a good idea: Quantitative modeling of the spread of ideas from epidemiological models. Physica A, 364:513-536, 2006.

[55] G. Szabó and A. L. Barabási. Network effects in service usage. arXiv:physics/061117nv1, 2006.

[56] Y. Moreno, M. Nekovee, and A. F. Pacheco. Dynamics of rumor spreading in complex networks. Phys. Rev. E, 69(6):066130, 2004.

[57] C. Castellano and V. Loreto. Statistical physics of social dynamics. Rev. Mod. Phys., 81(2):591, 2009.

[58] P. Van den Driessche and J. Watmough. Reproduction numbers and subthreshold endemic equilibria for compartmental models of disease transmission. Math. Biosci., 180(1):29-48, 2002.

[59] O. Diekmann, J. A. P. Heesterbeek, and M. G. Roberts. The construction of next-generation matrices for compartmental epidemic models. J. Roy. Soc. Interface, 7(47):873-885, 2010. 
[60] P. Grassberger. On the critical behavior of the general epidemic process and dynamical percolation. Math. Biosci., 63(2):157-172, 1983.

[61] L. M. Sander, C. P. Warren, I. M. Sokolov, C. Simon, and J. Koopman. Percolation on heterogeneous networks as a model for epidemics. Math. Biosci., 180(1-2):293-305, 2002.

[62] T. Gross and B. Blasius. Adaptive coevolutionary networks: a review. J. Roy. Soc. Interface, 5(20):259, 2008.

[63] C. Huepe, G. Zschaler, A. L. Do, and T. Gross. Adaptive-network models of swarm dynamics. N. J. Phys., 13(7):073022, 2011.

[64] I. D. Couzin, C. C. Ioannou, G. Demirel, T. Gross, C. J. Torney, A. Hartnett, L. Conradt, S. A. Levin, and N. E. Leonard. Uninformed individuals promote democratic consensus in animal groups. Science, 334(6062):15781580, 2011.

[65] C. Zhou and J. Kurths. Dynamical weights and enhanced synchronization in adaptive complex networks. Phys. Rev. Lett., 96(16):164102, 2006.

[66] S. Bornholdt and T. Rohlf. Topological evolution of dynamical networks: Global criticality from local dynamics. Phys. Rev. Lett., 84(26):6114-6117, 2000 .

[67] C. Meisel, A. Storch, S. Hallmeyer-Elgner, E. Bullmore, and T. Gross. Failure of adaptive self-organized criticality during epileptic seizure attacks. PLoS Comput. Biol., 8(1):e1002312, 2012.

[68] P. Holme and G. Ghoshal. Dynamics of networking agents competing for high centrality and low degree. Phys. Rev. Lett., 96(9):098701, 2006.

[69] M. G. Zimmermann, V. M. Eguíluz, M. San Miguel, and A. Spadaro. Applications of Simulations in Social Sciences. Hermes Science Publications, 2000 .

[70] A. L. Do, L. Rudolf, and T. Gross. Patterns of cooperation: fairness and coordination in networks of interacting agents. N. J. Phys., 12:063023, 2010 .

[71] J. Ito and K. Kaneko. Spontaneous structure formation in a network of chaotic units with variable connection strengths. Phys. Rev. Lett., 88(2):028701, 2001.

[72] T. Gross, C. J. D. D'Lima, and B. Blasius. Epidemic dynamics on an adaptive network. Phys. Rev. Lett., 96(20):208701, 2006. 
[73] F. Vazquez, V. M. Eguíluz, and M. S. Miguel. Generic absorbing transition in coevolution dynamics. Phys. Rev. Lett., 100(10):108702, 2008.

[74] T. Aoki and T. Aoyagi. Co-evolution of phases and connection strengths in a network of phase oscillators. Phys. Rev. Lett., 102(3):34101, 2009.

[75] L. B. Shaw and I. B. Schwartz. Fluctuating epidemics on adaptive networks. Phys. Rev. E, 77:066101, 2008.

[76] G. Rozhnova, A. Nunes, and A. J. McKane. Stochastic oscillations in models of epidemics on a network of cities. Phys. Rev. E, 84:051919, 2011.

[77] T. Rogers, W. Clifford-Brown, C. Mills, and T. Galla. Stochastic oscillations of adaptive networks: application to epidemic modelling. J. Stat. Mech., 2012(08):P08018, 2012.

[78] A. L. Do and T. Gross. Contact processes and moment closure on adaptive networks. Adaptive Networks, 2009.

[79] M. Boguñá and R. Pastor-Satorras. Epidemic spreading in correlated complex networks. Phys. Rev. E, 66(4):047104, 2002.

[80] V. M. Eguiluz and K. Klemm. Epidemic threshold in structured scale-free networks. Phys. Rev. Lett., 89(10):108701, 2002.

[81] G. Zschaler, A. Traulsen, and T. Gross. A homoclinic route to asymptotic full cooperation in adaptive networks and its failure. N. J. Phys., 12:093015, 2010.

[82] G. Demirel, R. Prizak, P. N. Reddy, and T. Gross. Cyclic dominance in adaptive networks. Eur. Phys. J. B, 84:541-548, 2011.

[83] D. Kimura and Y. Hayakawa. Coevolutionary networks with homophily and heterophily. Phys. Rev. E, 78(1):016103, 2008.

[84] L. B. Shaw and I. B. Schwartz. Enhanced vaccine control of epidemics in adaptive networks. Phys. Rev. E, 81:046120, 2010.

[85] S. Risau-Gusman and D. H. Zanette. Contact switching as a control strategy for epidemic outbreaks. J. Theor. Biol., 257(1):52-60, 2009.

[86] I. U. A. Kuznetsov. Elements of applied bifurcation theory. Springer Verlag, 1998.

[87] D. J. Watts. The "new" science of networks. Ann. Rev. Soc., 30:243-270, 2004 . 
[88] A. L. Barabási and J. Frangos. Linked: The New Science Of Networks. Perseus Publishing, 2002.

[89] J. Scott. Social network analysis. Sociology, 22(1):109-127, 1988.

[90] J. A. Barnes. Graph theory and social networks: A technical comment on connectedness and connectivity. Sociology, 3(2):215-232, 1969.

[91] P. A. Grabowicz, J. J. Ramasco, and V. M. Eguiluz. Dynamics in online social networks. arXiv:1210.0808, 2012.

[92] R. Kumar, J. Novak, and A. Tomkins. Structure and evolution of online social networks. Link Mining: Models, Algorithms, and Applications, 2010 .

[93] D. Centola. The spread of behavior in an online social network experiment. Science, 329(5996):1194-1197, 2010.

[94] N. Eagle, A. S. Pentland, and D. Lazer. Inferring friendship network structure by using mobile phone data. P. Natl. Acad. Sci., 106(36):1527415278, 2009.

[95] C. A. Hidalgo and C. Rodriguez-Sickert. The dynamics of a mobile phone network. Physica A, 387(12):3017-3024, 2008.

[96] A. Mislove, M. Marcon, K. P. Gummadi, P. Druschel, and B. Bhattacharjee. Measurement and analysis of online social networks. Proc. 7th conf. on Internet measurement, 2007.

[97] G. Kossinets and D. J. Watts. Empirical analysis of an evolving social network. Science, 311(5757):88-90, 2006.

[98] H. Ohtsuki, C. Hauert, E. Lieberman, and M. A. Nowak. A simple rule for the evolution of cooperation on graphs and social networks. Nature, 441(7092):502-505, 2006.

[99] L. DallAsta, A. Baronchelli, A. Barrat, and V. Loreto. Nonequilibrium dynamics of language games on complex networks. Phys. Rev. E, 74(3):036105, 2006.

[100] P. Holme and M. E. J. Newman. Nonequilibrium phase transition in the coevolution of networks and opinions. Phys. Rev. E, 74(5):056108, 2006.

[101] S. Gil and D. H. Zanette. Coevolution of agents and networks: Opinion spreading and community disconnection. Phys. Lett. A, 356(2):89-94, 2006 . 
[102] R. Hegselmann and U. Krause. Opinion dynamics and bounded confidence: models, analysis and simulation. J. Artif. Soc. Soc. Simul., 5(3), 2002 .

[103] G. Weisbuch, G. Deffuant, F. Amblard, and J. P. Nadal. Meet, discuss, and segregate! Complexity, 7(3):55-63, 2002.

[104] P. Chen and S. Redner. Consensus formation in multi-state majority and plurality models. J. Phys. A, 38:7239, 2005.

[105] B. Kozma and A. Barrat. Consensus formation on adaptive networks. Phys. Rev. E, 77(1):016102, 2008.

[106] G. Deffuant, D. Neau, F. Amblard, and G. Weisbuch. Mixing beliefs among interacting agents. Adv. Complex Sys., 3(4):87-98, 2000.

[107] R. Hegselmann and U. Krause. Opinion dynamics driven by various ways of averaging. Comp. Econ., 25(4):381-405, 2005.

[108] E. Ben-Naim. Opinion dynamics: rise and fall of political parties. Europhys. Lett., 69:671, 2005.

[109] C. Castellano, M. Marsili, and A. Vespignani. Nonequilibrium phase transition in a model for social influence. Phys. Rev. Lett., 85:3536-3539, 2000.

[110] F. Vazquez and S. Redner. Non-monotonicity and divergent time scale in axelrod model dynamics. Europhys. Lett., 78:18002, 2007.

[111] J. L. Herrera, M. G. Cosenza, K. Tucci, and J. C. González-Avella. General coevolution of topology and dynamics in networks. Europhys. Lett., 95:58006, 2011.

[112] R. Durrett, J. P. Gleeson, A. L. Lloyd, P. J. Mucha, F. Shi, D. Sivakoff, J. E. S. Socolar, and C. Varghese. Graph fission in an evolving voter model. P. Natl. Acad. Sci., 2012.

[113] P. L. Krapivsky and S. Redner. Dynamics of majority rule in two-state interacting spin systems. Phys. Rev. Lett., 90(23):238701, 2003.

[114] F. Vazquez and V. M. Eguíluz. Analytical solution of the voter model on uncorrelated networks. N. J. Phys., 10:063011, 2008.

[115] G. Demirel, F. Vazquez, G. A. Böhme, and T. Gross. Moment closure approximations for discrete adaptive networks. arXiv:1211.0449, 2012.

[116] G. A. Böhme and T. Gross. Analytical calculation of fragmentation transitions in adaptive networks. Phys. Rev. E, 83(3):035101, 2011. 
[117] G. Zschaler, G. A. Böhme, M. Seißinger, C. Huepe, and T. Gross. Early fragmentation in the adaptive voter model on directed networks. Phys. Rev. E, 85:046107, 2012.

[118] G. A. Böhme and T. Gross. Fragmentation transitions in multistate voter models. Phys. Rev. E, 85:066117, 2012.

[119] D. H. Zanette and S. Gil. Opinion spreading and agent segregation on evolving networks. Physica D, 224(1-2):156-165, 2006.

[120] T. M. Liggett. Stochastic interacting systems: contact, voter, and exclusion processes. Springer Verlag, 1999.

[121] G. Demirel. PhD Thesis, 2012.

[122] S. R. Broadbent and J. M. Hammersley. Percolation processes i. crystals and mazes. Proc. Cambridge phil. soc., 53(3):629-641, 1957.

[123] M. A. Serrano and M. Boguñá. Percolation and epidemic thresholds in clustered networks. Phys. Rev. Lett., 97:088701, 2006.

[124] I. Derényi, G. Palla, and T. Vicsek. Clique percolation in random networks. Phys. Rev. Lett., 94:160202, 2005.

[125] D. Achlioptas, R. M. D'Souza, and J. Spencer. Explosive percolation in random networks. Science, 323(5920):1453-1455, 2009.

[126] J. Shao, S. Havlin, and H. E. Stanley. Dynamic opinion model and invasion percolation. Phys. Rev. Lett., 103:018701, 2009.

[127] D. S. Callaway, M. E. J. Newman, S. H. Strogatz, and Duncan J. Watts. Network robustness and fragility: Percolation on random graphs. Phys. Rev. Lett., 85:5468-5471, 2000.

[128] C. Moore and M. E. J. Newman. Exact solution of site and bond percolation on small-world networks. Phys. Rev. E, 62:7059-7064, 2000.

[129] D. Juher, J. Ripoll, and J. Saldaña. Outbreak analysis of an sis epidemic model with rewiring. J. Math. Biol., pages 1-22, 2012.

[130] Y. Wang, D. Chakrabarti, C. Wang, and C. Faloutsos. Epidemic spreading in real networks: An eigenvalue viewpoint. Proc. 22nd Int. Symp. on Reliable Distributed Systems, 2003.

[131] J. C. Kamgang and G. Sallet. Computation of threshold conditions for epidemiological models and global stability of the disease-free equilibrium (dfe). Math. Biosci., 213(1):1-12, 2008. 
[132] M. E. J. Newman, S. H. Strogatz, and D. J. Watts. Random graphs with arbitrary degree distributions and their applications. Phys. Rev. E, 64(2):026118, 2001.

[133] R. S. Varga. Matrix iterative analysis. Springer Verlag, 2010.

[134] E. Deutsch. Bounds for the perron root of a nonnegative irreducible partitioned matrix. Pac. J. Math., 92(1):49-56, 1981.

[135] Y. Y. Ahn, S. Han, H. Kwak, S. Moon, and H. Jeong. Analysis of topological characteristics of huge online social networking services. Proc. 16th int. conf. on WorldWideWeb, 2007.

[136] N. Masuda and S. Redner. Can partisan voting lead to truth? J. Stat. Mech., 2011:L02002, 2011.

[137] F. Vazquez and S. Redner. Ultimate fate of constrained voters. J. Phys. A, 37:8479, 2004 .

[138] C. Castellano, M. A. Muñoz, and R. Pastor-Satorras. Nonlinear q-voter model. Phys. Rev. E, 80(4):041129, 2009.

[139] R. L. Lindeman. The trophic-dynamic aspect of ecology. Ecology, 23(4):399-417, 1942.

[140] R. T. Paine. Food web complexity and species diversity. Am. Nat., 100:6575, 1966.

[141] G. A. Polis and D. R. Strong. Food web complexity and community dynamics. Am. Nat., 147:813-846, 1996.

[142] J. A. Dunne, R. J. Williams, and N. D. Martinez. Food-web structure and network theory: the role of connectance and size. P. Natl. Acad. Sci., 99(20):12917, 2002.

[143] S. L. Pimm, J. H. Lawton, and J. E. Cohen. Food web patterns and their consequences. Nature, 350(6320):669-674, 1991.

[144] M. Pascual and J. A. Dunne. Ecological networks: linking structure to dynamics in food webs. Oxford University Press, 2006.

[145] N. D. Martinez. Artifacts or attributes? effects of resolution on the little rock lake food web. Ecol. Monogr., 61:367-392, 1991.

[146] G.A. Polis. Complex trophic interactions in deserts: an empirical critique of food-web theory. Am. Nat., 138:123-155, 1991. 
[147] L. Goldwasser and J. Roughgarden. Construction and analysis of a large caribbean food web. Ecology, 74:1216-1233, 1993.

[148] S. J. Hall and D. Raffaelli. Food-web patterns: lessons from a species-rich web. J. Anim. Ecol., 60:823-841, 1991.

[149] K. McCann, A. Hastings, and G. R. Huxel. Weak trophic interactions and the balance of nature. Nature, 395(6704):794-798, 1998.

[150] U. Brose, R. J. Williams, and N. D. Martinez. Allometric scaling enhances stability in complex food webs. Ecol. Lett., 9(11):1228-1236, 2006.

[151] J. Vandermeer. Omnivory and the stability of food webs. J. Theor. Biol., 238(3):497-504, 2006.

[152] T. Gross, L. Rudolf, S. A. Levin, and U. Dieckmann. Generalized models reveal stabilizing factors in food webs. Science, 325(5941):747-750, 2009.

[153] B. Kartascheff, C. Guill, B. Drossel, et al. Positive complexity-stability relations in food web models without foraging adaptation. J. Theor. Biol., 259(1), 2009.

[154] R. Levins. Some demographic and genetic consequences of environmental heterogeneity for biological control. Bull. Entomol. Soc. Am., 15(3):237$240,1969$.

[155] I. Hanski. Metapopulation dynamics. Nature, 396(6706):41-49, 1998.

[156] P. Pillai, M. Loreau, and A. Gonzalez. A patch-dynamic framework for food web metacommunities. Theor. Ecol., 3(4):223-237, 2010.

[157] P. Pillai, A. Gonzalez, and M. Loreau. Metacommunity theory explains the emergence of food web complexity. P. Natl. Acad. Sci., 108(48):1929319298, 2011.

[158] P. Pillai, A. Gonzalez, and M. Loreau. Evolution of dispersal in a predatorprey metacommunity. Am. Nat., 179(2):204-216, 2012.

[159] F. R. Adler and B. Nuernberger. Persistence in patchy irregular landscapes. Theor. Pop. Biol., 45(1):41-75, 1994.

[160] O. Ovaskainen and I. Hanski. Spatially structured metapopulation models: global and local assessment of metapopulation capacity. Theor. Pop. Biol., 60(4):281-302, 2001. 
[161] A. V. Goltsev, S. N. Dorogovtsev, J. G. Oliveira, and J. F. F. Mendes. Localization and spreading of diseases in complex networks. arXiv:1202.4411, 2012.

[162] C. Castellano and R. Pastor-Satorras. Thresholds for epidemic spreading in networks. Phys. Rev. Lett., 105(21):218701, 2010.

[163] I. Hanski and O. Ovaskainen. The metapopulation capacity of a fragmented landscape. Nature, 404(6779):755-758, 2000.

[164] B. Karrer and M. E. J. Newman. Competing epidemics on complex networks. Phys. Rev. E, 84(3):036106, 2011.

[165] M. E. J. Newman. Threshold effects for two pathogens spreading on a network. Phys. Rev. Lett., 95(10):108701, 2005.

[166] N. Masuda and N. Konno. Multi-state epidemic processes on complex networks. J. Theor. Biol., 243(1):64-75, 2006.

[167] Y. Y. Ahn, H. Jeong, N. Masuda, and J. D. Noh. Epidemic dynamics of two species of interacting particles on scale-free networks. Phys. Rev. E, 74(6):066113, 2006.

[168] A. Arenas, A. Díaz-Guilera, J. Kurths, Y. Moreno, and C. Zhou. Synchronization in complex networks. Phys. Rep., 469(3):93-153, 2008. 



\section{Acknowledgements}

First, I would like to thank Dr. Thilo Gross for supervising this thesis. In particular, I would like to thank him for proposing interesting projects and providing helpful input and comments in the course of the work on these projects.

Second, I would like to thank Prof. Dr. Frank Jülicher for supervising this thesis and for giving me the great opportunity to conduct research in the Biological Physics division at the Max Planck Institute for the Physics of Complex Systems. The excellent working conditions and the inspiring environment of the institute contributed beneficially to the accomplishment of this work.

Next, I would like to thank all my colleagues from the group "Dynamics of Biological Networks". In particular, I thank Gerd Zschaler who was extremely helpful and patient concerning all kinds of programming-related questions. Moreover, he provided the $\mathrm{C}++$ library Largenet, which is the basis for all network simulations done in this work. Further, I thank Güven Demirel for many fruitful discussions and collaborations, and Ly Do for proof-reading an earlier version of this thesis and providing many helpful comments.

I thank all my collaborators. It was a pleasure to work on common projects with Federico Vazquez, Güven Demirel and Gerd Zschaler.

Furthermore, I would like to thank my office mate Rick Mukherjee. It was really fun and entertaining to share an office with him.

Last but not least, I thank my boyfriend Matthias Benndorf for his constant support and encouragement, especially during the tedious periods in the process of this work. 



\section{Versicherung}

Hiermit versichere ich, dass ich die vorliegende Arbeit ohne unzulässige Hilfe Dritter und ohne Benutzung anderer als der angegebenen Hilfsmittel angefertigt habe; die aus fremden Quellen direkt oder indirekt bernommenen Gedanken sind als solche kenntlich gemacht. Die Arbeit wurde bisher weder im Inland noch im Ausland in gleicher oder ähnlicher Form einer anderen Prüfungsbehörde vorgelegt. Ich erkenne die Promotionsordnung der Fakultät für Mathematik und Naturwissenschaften der Technischen Universität Dresden an.

Die vorliegende Arbeit wurde im Zeitraum 04/2010 - 12/2012 am Max-PlanckInstitut für Physik komplexer Systeme unter der Betreuung von Dr. Thilo Gross und Prof. Dr. Frank Jülicher angefertigt.

Dresden, Dezember 2012

Gesa Böhme 